Origins of Internal Regioselectivity in Copper-Catalyzed Borylation of Terminal Alkynes

Takumi Tsushima, Hideya Tanaka, Kazuki, Nakanishi, Masaaki Nakamoto, and Hiroto Yoshida*

Graduate School of Advanced Science and Engineering, Hiroshima University, Higashi-Hiroshima 739-8526, Japan

e-mail: yhiroto@hiroshima-u.ac.jp

Table of Contents

1. General Remarks

2. Optimization of Reaction Conditions

3. Computational Details (FIA)

4. Computational Details $\left(\% V_{\text {bur }}\right)$

5. Mechanistic Studies on a Catalytic Cycle

6. Experimental Procedures and Characterization of Products

7. X-ray Crystallographic Analyses

8. References

S38

9. ${ }^{1} \mathrm{H}$ and ${ }^{13} \mathrm{C}$ and ${ }^{11} \mathrm{~B}$ and Spectra of Products 


\section{General Remarks.}

All manipulations of oxygen- and moisture-sensitive materials were conducted with a standard Schlenk technique under an argon atmosphere. Cross-coupling reactions was carried out with a microwave reactor (Biotage Initiator). Nuclear magnetic resonance spectra were taken on a Varian System $500\left({ }^{1} \mathrm{H}, 500\right.$ $\left.\mathrm{MHz} ;{ }^{13} \mathrm{C}, 125 \mathrm{MHz} ;{ }^{11} \mathrm{~B}, 160 \mathrm{MHz}\right)$ spectrometer using residual proton in DMSO- $d_{6}\left({ }^{1} \mathrm{H}, \delta=2.50\right)$, residual chloroform $\left({ }^{1} \mathrm{H}, \delta=7.26\right)$ or $\mathrm{CDCl}_{3}\left({ }^{13} \mathrm{C}, \delta=77.0\right)$ as an internal standard and boron trifluoride diethyl etherate $\left({ }^{11} \mathrm{~B}, \delta=0.00\right)$ as an external standard. ${ }^{1} \mathrm{H}$ NMR data are reported as follows: chemical shift, multiplicity ( $\mathrm{s}=$ singlet, $\mathrm{d}=$ doublet, $\mathrm{t}=$ triplet, $\mathrm{q}=$ quartet, $\mathrm{sep}=$ septet, $\mathrm{m}=$ multiplet), coupling constants $(\mathrm{Hz})$, integration. High-resolution mass spectra were obtained with a Thermo Fisher Scientific LTQ Orbitrap XL spectrometer. Melting points were measured with Yanaco Micro Melting Point apparatus and uncorrected. Preparative recycling gel permeation chromatography was performed with GL Science PU 614 equipped with Shodex GPC H-2001L and -2002L columns (toluene as an eluent). Column chromatography was carried out using Merck Kieselgel 60. SIPrCuCl, ${ }^{1}$ IMesCuCl, ${ }^{1}$

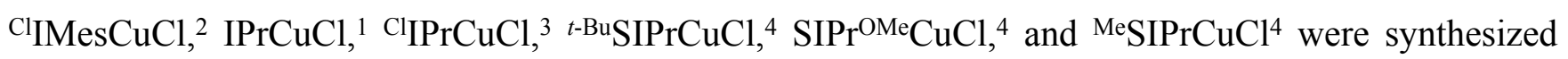
according to literature procedures. Unless otherwise noted, commercially available reagents were used without purification. All solvents were dried over activated molecular sieves $3 \AA$.

\section{Optimization of Reaction Conditions}

A Schlenk tube equipped with a magnetic stirring bar was charged with $\mathrm{NHCCuCl}(7.5 \mu \mathrm{mol}), t-\mathrm{BuOK}$ $(1.0 \mathrm{M}$ solution in THF, $9.0 \mu \mathrm{mol})$ and solvent $(0.5 \mathrm{~mL})$ before the mixture was stirred at room temperature for $10 \mathrm{~min}$. To the mixture was added (pin)B-B(aam) $(0.18 \mathrm{mmol}), \mathrm{MeOH}(0.15 \times \mathrm{X} \mathrm{mmol})$ and 1-octyne $(0.15 \mathrm{mmol})$, and the resulting mixture was stirred at $50{ }^{\circ} \mathrm{C}$ for $3 \mathrm{~h}$. The mixture was diluted with ethyl acetate and filtered through a Celite plug. The organic solution was washed with brine, dried over $\mathrm{Na}_{2} \mathrm{SO}_{4}$, and evaporated. Then yield and regioselectivity were analyzed by ${ }^{1} \mathrm{H}$ NMR. 
Table S1. Optimization of Reaction Conditions ${ }^{a}$

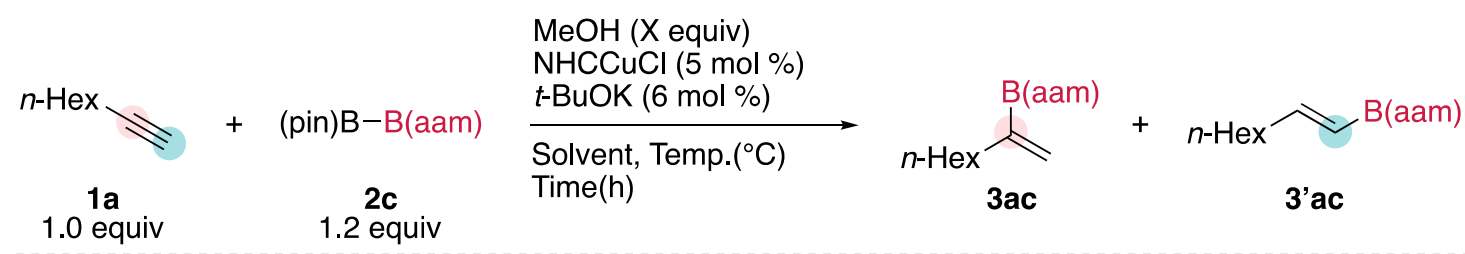

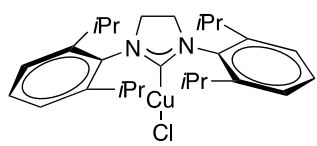

SIPrCuCl

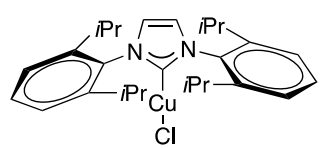

IPrCuCl

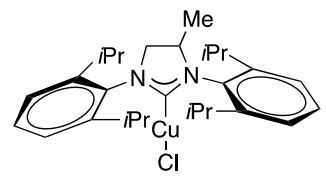

MesIPrCuCl

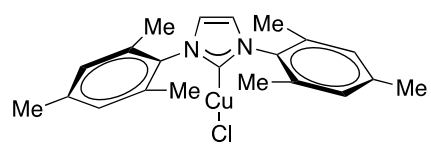

IMesCuCl

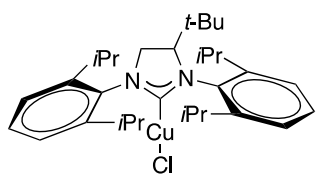

t-BuSIPrCuCl

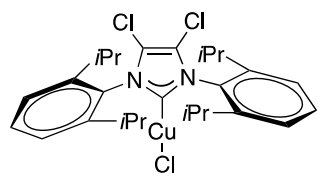

CliPrCuCl

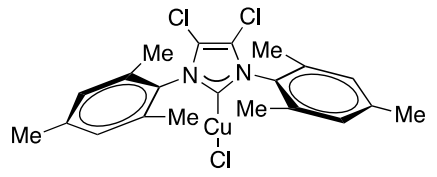

ClimesCuCl

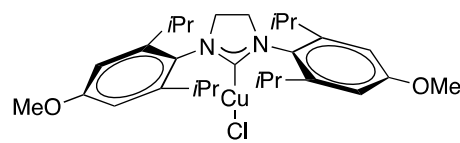

$\mathrm{SIPrOMe} \mathrm{CuCl}$

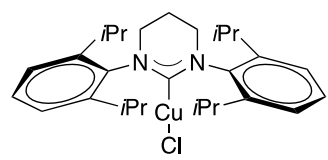

6DippCuCl

\begin{tabular}{|c|c|c|c|c|c|c|c|}
\hline Entry & Temp. $\left({ }^{\circ} \mathrm{C}\right)$ & $\begin{array}{c}\mathrm{MeOH} \\
\text { (X equiv) }\end{array}$ & Time(h) & Solvent & $\mathrm{NHCCuCl}$ & $\begin{array}{c}\text { NMR } \\
\text { yield(\%) }\end{array}$ & $b: l$ \\
\hline 1 & r.t. & 1.5 & 24 & THF & SIPrCuCl & 87 & $89: 11$ \\
\hline 2 & 50 & 1.5 & 3 & THF & $\mathrm{SIPrCuCl}$ & 87 & $89: 11$ \\
\hline 3 & 50 & 3.0 & 3 & THF & $\mathrm{SIPrCuCl}$ & 91 & $89: 11$ \\
\hline 4 & 50 & 3.0 & 3 & DMF & SIPrCuCl & 81 & $71: 29$ \\
\hline 5 & 50 & 3.0 & 3 & $\mathrm{MeCN}$ & $\mathrm{SIPrCuCl}$ & 72 & $78: 22$ \\
\hline 6 & 50 & 3.0 & 3 & toluene & $\mathrm{SIPrCuCl}$ & 73 & $87: 13$ \\
\hline 7 & 50 & 3.0 & 3 & CPME & $\mathrm{SIPrCuCl}$ & 75 & $87: 13$ \\
\hline 8 & 50 & 3.0 & 3 & 1,4-dioxane & $\mathrm{SIPrCuCl}$ & 92 & 91:9 \\
\hline 9 & 50 & 3.0 & 3 & 1,4-dioxane & IMesCuCl & 42 & $40: 60$ \\
\hline 10 & 50 & 3.0 & 3 & 1,4-dioxane & ${ }^{\mathrm{Cl}} \mathrm{IMes} \mathrm{CuCl}$ & 46 & $46: 54$ \\
\hline 11 & 50 & 3.0 & 3 & 1,4-dioxane & $\mathrm{IPrCuCl}$ & 84 & $88: 12$ \\
\hline 12 & 50 & 3.0 & 3 & 1,4-dioxane & ${ }_{t-\mathrm{Bu}} \mathrm{SIPrCuCl}$ & 85 & $90: 10$ \\
\hline 13 & 50 & 3.0 & 3 & 1,4-dioxane & $\mathrm{SIPr}^{\mathrm{OMe}} \mathrm{CuCl}$ & 79 & 91:9 \\
\hline 14 & 50 & 3.0 & 3 & 1,4-dioxane & ${ }^{\mathrm{Me}} \mathrm{SIPrCuCl}$ & 93 & $91: 9$ \\
\hline 15 & 50 & 3.0 & 3 & 1,4-dioxane & ${ }^{\mathrm{Cl}} \mathrm{IPrCuCl}$ & 77 & $>99: 1$ \\
\hline 16 & 50 & 3.0 & 3 & 1,4-dioxane & 6DippCuCl & 96 & $>99: 1$ \\
\hline $17^{b}$ & 50 & 3.0 & 3 & 1,4-dioxane & 6DippCuCl & 60 & $>99: 1$ \\
\hline
\end{tabular}

${ }^{a} \mathbf{1 a}(0.15 \mathrm{mmol}=1.0$ equiv $), 2 \mathrm{c}(0.18 \mathrm{mmol}), \mathrm{MeOH}(\mathrm{X}$ equiv $), \mathrm{NHCCuCl}(7.5 \mu \mathrm{mol}), t-\mathrm{BuOK}(1.0 \mathrm{M}$ 
THF solution, $9 \mu \mathrm{mol})$, Solvent $(0.5 \mathrm{~mL})$

${ }^{b} 6 \mathrm{DippCuCl}=2 \mathrm{~mol} \%$ 


\section{Computational Details (FIA)}

Geometry optimization and vibrational frequency calculation of $\mathrm{COF}_{2}$ and $\mathrm{PhBX}_{2}$ as well as their fluoride adducts were conducted by using B3LYP ${ }^{5-7}$ with $6-31+\mathrm{G}(\mathrm{d}, \mathrm{p})^{8}$ basis set (this level of theory is used for FIA calculation in a reference ${ }^{9}$ ) on Gaussian 16 Rev. B01 program..$^{10}$ All optimized structures were confirmed to be local minima by verifying the absence of imaginary frequencies. The values of fluoride ion affinity (FIA) were obtained by using the experimental FIA value of $\mathrm{COF}_{2}(209 \mathrm{~kJ} / \mathrm{mol})$ as an anchor point in a (pseudo-)isodesmic reaction at the same level of theory. ${ }^{11,12}$

Table S2. DFT Calculation of FIA

$$
\begin{aligned}
\mathrm{PhBX}_{2}+\mathrm{COF}_{3}^{-} \stackrel{\Delta \mathrm{H}^{1}}{\longrightarrow}\left[\mathrm{PhBX}_{2}-\mathrm{F}\right]^{-}+\mathrm{COF}_{2} \\
\mathrm{COF}_{3}^{-} \stackrel{\Delta \mathrm{H}^{2}=209(\mathrm{~kJ} / \mathrm{mol})}{\longrightarrow} \mathrm{COF}_{2}+\mathrm{F}^{-}
\end{aligned}
$$

$$
\mathrm{PhBX}_{2}+\mathrm{F}^{-} \stackrel{\mathrm{FIA}=\Delta \mathrm{H}^{1}-\Delta \mathrm{H}^{2}}{\longrightarrow}\left[\mathrm{PhBX}_{2}-\mathrm{F}\right]^{-}
$$

\begin{tabular}{|c|c|c|c|}
\hline $\mathbf{P h}-\mathrm{BX}_{2}$ & H (hartree) & $\Delta H^{1}(\mathrm{~kJ} / \mathrm{mol})$ & FIA $(\mathrm{kJ} / \mathrm{mol})$ \\
\hline $\mathrm{COF}_{2}$ & -313.010511 & & \\
\hline $\mathrm{COF}_{3}^{-}$ & -412.949407 & & \\
\hline $\mathrm{PhB}(\mathrm{dan})$ & -751.795662 & -6.5 & -215.5 \\
\hline $\mathrm{PhB}(\mathrm{dan})-\mathrm{F}^{-}$ & -851.737037 & & \\
\hline $\mathrm{PhB}($ mdan $)$ & -830.343309 & -20.4 & -229.4 \\
\hline $\mathrm{PhB}($ mdan $)-\mathrm{F}^{-}$ & -930.289966 & & \\
\hline $\mathrm{PhB}(\mathrm{aam})$ & -711.538401 & -27.1 & -236.1 \\
\hline $\mathrm{PhB}(\mathrm{aam})-\mathrm{F}^{-}$ & -811.487635 & & \\
\hline $\mathrm{PhB}($ pin $)$ & -642.700370 & 12.5 & -196.5 \\
\hline $\mathrm{PhB}($ pin $)-\mathrm{F}^{-}$ & -742.634512 & & \\
\hline
\end{tabular}

FIA at the B3LYP/6-31+G(d,p) level of theory anchored to the $\mathrm{COF}_{3}{ }^{-} / \mathrm{COF}_{2}+\mathrm{F}^{-}$reaction 
$\mathrm{COF}_{2}$

Charge $=0 ;$ Multiplicity $=1$

$\begin{array}{lrrr}\text { C } & -0.00000141 & 0.14557588 & -0.00000000 \\ \text { F } & 1.07108750 & -0.63745435 & 0.00000000 \\ \text { O } & -0.00001391 & 1.32511571 & -0.00000000 \\ \text { F } & -1.07107419 & -0.63747687 & -0.00000000\end{array}$

$\mathrm{COF}_{3}^{-}$

Charge $=-1 ;$ Multiplicity $=1$

$\begin{array}{lccc}\text { C } & -0.00095805 & -0.00023674 & 0.19584359 \\ \text { F } & -0.85121142 & 0.95919112 & -0.46646023 \\ \text { O } & -0.00706168 & -0.00212568 & 1.41795255 \\ \text { F } & 1.25922027 & 0.26052743 & -0.45704805 \\ \text { F } & -0.40109310 & -1.21767123 & -0.46745638\end{array}$


$\mathrm{PhB}$ (dan)

Charge $=0 ;$ Multiplicity $=1$

\begin{tabular}{|c|c|c|c|}
\hline$C$ & 4.68781919 & 1.06096369 & -0.57790182 \\
\hline $\mathrm{C}$ & 5.39055903 & 0.00000033 & -0.00000062 \\
\hline $\mathrm{C}$ & 4.68782000 & -1.06096331 & 0.57790103 \\
\hline ' & 3.29113879 & -1.05785864 & 0.57329034 \\
\hline $\mathrm{C}$ & 2.55823474 & -0.00000032 & 0.00000022 \\
\hline & 3.29113799 & 1.05785832 & -0.57329036 \\
\hline B & 0.98968935 & -0.00000042 & 0.00000134 \\
\hline $\mathrm{N}$ & 0.22814020 & -1.20265401 & -0.10080543 \\
\hline $\mathrm{N}$ & 0.22814044 & 1.20265345 & 0.10080651 \\
\hline $\mathrm{C}$ & -1.17026138 & -1.24035160 & -0.10479471 \\
\hline $\mathrm{C}$ & -1.17026111 & 1.24035140 & 0.10479521 \\
\hline $\mathrm{C}$ & -1.87408823 & -2.43154897 & -0.21108111 \\
\hline $\mathrm{C}$ & -3.28537373 & -2.42219239 & -0.21317457 \\
\hline $\mathrm{C}$ & -3.99171809 & -1.24211549 & -0.10978504 \\
\hline $\mathrm{C}$ & -3.30685688 & 0.00000014 & -0.00000004 \\
\hline $\mathrm{C}$ & -1.87539665 & -0.00000004 & 0.00000037 \\
\hline $\mathrm{C}$ & -3.99171784 & 1.24211595 & 0.10978444 \\
\hline $\mathrm{C}$ & -3.28537323 & 2.42219270 & 0.21317412 \\
\hline $\mathrm{C}$ & -1.87408773 & 2.43154897 & 0.21108091 \\
\hline $\mathrm{H}$ & 5.22690242 & 1.88633038 & -1.03465333 \\
\hline H & 6.47691373 & 0.00000061 & -0.00000086 \\
\hline $\mathrm{H}$ & 5.22690387 & -1.88632969 & 1.03465237 \\
\hline $\mathrm{H}$ & 2.76431170 & -1.88499205 & 1.04427268 \\
\hline $\mathrm{H}$ & 2.76431026 & 1.88499162 & -1.04427219 \\
\hline $\mathrm{H}$ & 0.68415199 & -2.09453518 & -0.23339377 \\
\hline $\mathrm{H}$ & 0.68415244 & 2.09453477 & 0.23339313 \\
\hline $\mathrm{H}$ & -1.33500696 & -3.37194937 & -0.29202703 \\
\hline $\mathrm{H}$ & -3.81701244 & -3.36591032 & -0.29737999 \\
\hline $\mathrm{H}$ & -5.07773476 & -1.24525192 & -0.11149896 \\
\hline $\mathrm{H}$ & -5.07773451 & 1.24525266 & 0.11149770 \\
\hline & -3.81701175 & 3.36591079 & 0.29737901 \\
\hline H & -1.33500627 & 3.37194928 & 0.29202671 \\
\hline
\end{tabular}


$\mathrm{PhB}($ dan $)-\mathrm{F}^{-}$

Charge $=-1 ;$ Multiplicity $=1$

\begin{tabular}{|c|c|c|c|}
\hline$C$ & 3.91519333 & 0.00001183 & -1.84533419 \\
\hline $\mathrm{C}$ & 5.06234026 & 0.00004456 & -1.04201748 \\
\hline$C$ & 4.91889501 & 0.00004224 & 0.34853780 \\
\hline ' & 3.64099265 & 0.00000787 & 0.92367456 \\
\hline $\mathrm{C}$ & 2.47085883 & -0.00002433 & 0.14256176 \\
\hline $\mathrm{C}$ & 2.64746530 & -0.00002206 & -1.25526465 \\
\hline$B$ & 0.97209428 & -0.00005385 & 0.80563277 \\
\hline $\mathrm{N}$ & 0.15970732 & -1.22133227 & 0.37257157 \\
\hline$N$ & 0.15972222 & 1.22127810 & 0.37269404 \\
\hline $\mathrm{C}$ & -1.18356920 & -1.24421458 & 0.13172314 \\
\hline $\mathrm{C}$ & -1.18353581 & 1.24420658 & 0.13177627 \\
\hline $\mathrm{C}$ & -1.89868951 & -2.44649952 & -0.02036791 \\
\hline $\mathrm{C}$ & -3.27615476 & -2.43149939 & -0.29274489 \\
\hline $\mathrm{C}$ & -3.97775044 & -1.24477423 & -0.42321158 \\
\hline $\mathrm{C}$ & -3.30053514 & 0.00003256 & -0.28355993 \\
\hline $\mathrm{C}$ & -1.89493949 & 0.00000771 & -0.00059590 \\
\hline $\mathrm{C}$ & -3.97771225 & 1.24486174 & -0.42319112 \\
\hline $\mathrm{C}$ & -3.27607860 & 2.43156324 & -0.29270537 \\
\hline $\mathrm{C}$ & -1.89861672 & 2.44651696 & -0.02031376 \\
\hline$F$ & 1.14170377 & -0.00012850 & 2.25996706 \\
\hline $\mathrm{H}$ & 4.01168896 & 0.00001263 & -2.92984986 \\
\hline $\mathrm{H}$ & 6.05182263 & 0.00007134 & -1.49492827 \\
\hline $\mathrm{H}$ & 5.80222126 & 0.00006498 & 0.98544137 \\
\hline $\mathrm{H}$ & 3.53645819 & 0.00000426 & 2.00565622 \\
\hline $\mathrm{H}$ & 1.76422370 & -0.00004965 & -1.89116798 \\
\hline . & 0.58042329 & -2.13365670 & 0.48837064 \\
\hline 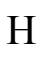 & 0.58048194 & 2.13358497 & 0.48846445 \\
\hline $\mathrm{H}$ & -1.36982870 & -3.39220811 & 0.07972457 \\
\hline $\mathrm{H}$ & -3.79996838 & -3.38024471 & -0.40085024 \\
\hline H & -5.04438233 & -1.24695042 & -0.63420503 \\
\hline $\mathrm{H}$ & -5.04434374 & 1.24707550 & -0.63418574 \\
\hline $\mathrm{H}$ & -3.79986141 & 3.38032641 & -0.40080385 \\
\hline $\mathrm{H}$ & -1.36972838 & 3.39220730 & 0.07980644 \\
\hline
\end{tabular}


$\mathrm{PhB}$ (mdan)

Charge $=0 ;$ Multiplicity $=1$

\begin{tabular}{|c|c|c|c|}
\hline C & 4.57735358 & 0.00018801 & 1.20747134 \\
\hline $\mathrm{C}$ & 5.28107797 & 0.00000002 & -0.00000025 \\
\hline $\mathrm{C}$ & 4.57735328 & -0.00018805 & -1.20747166 \\
\hline $\mathrm{C}$ & 3.17920971 & -0.00017857 & -1.20341058 \\
\hline$C$ & 2.44843763 & 0.00000101 & 0.00000012 \\
\hline $\mathrm{C}$ & 3.17921001 & 0.00017896 & 1.20341063 \\
\hline B & 0.86349631 & 0.00000102 & 0.00000029 \\
\hline $\mathrm{N}$ & 0.13075925 & 1.23212959 & 0.00010800 \\
\hline N & 0.13076095 & -1.23212833 & -0.00010771 \\
\hline $\mathrm{C}$ & -1.27600767 & 1.25096596 & -0.00000596 \\
\hline $\mathrm{C}$ & -1.27600639 & -1.25096681 & 0.00000614 \\
\hline $\mathrm{C}$ & -1.99973490 & 2.44159387 & -0.00012522 \\
\hline $\mathrm{C}$ & -3.41042680 & 2.42500756 & -0.00016482 \\
\hline $\mathrm{C}$ & -4.10711071 & 1.23885753 & -0.00007647 \\
\hline $\mathrm{C}$ & -3.41043625 & -0.00000109 & -0.00000004 \\
\hline $\mathrm{C}$ & -1.97618210 & -0.00000072 & 0.00000007 \\
\hline $\mathrm{C}$ & -4.10711004 & -1.23886003 & 0.00007627 \\
\hline $\mathrm{C}$ & -3.41042540 & -2.42500956 & 0.00016468 \\
\hline $\mathrm{C}$ & -1.99973345 & -2.44159510 & 0.00012524 \\
\hline $\mathrm{C}$ & 0.80717301 & -2.52870537 & -0.00006094 \\
\hline $\mathrm{C}$ & 0.80716698 & 2.52870899 & 0.00006103 \\
\hline $\mathrm{H}$ & 5.11565722 & 0.00033639 & 2.15143360 \\
\hline $\mathrm{H}$ & 6.36743521 & -0.00000029 & -0.00000038 \\
\hline $\mathrm{H}$ & 5.11565667 & -0.00033697 & -2.15143406 \\
\hline $\mathrm{H}$ & 2.65009707 & -0.00032472 & -2.15405237 \\
\hline $\mathrm{H}$ & 2.65009762 & 0.00032484 & 2.15405255 \\
\hline $\mathrm{H}$ & -1.49375215 & 3.39733870 & -0.00020595 \\
\hline $\mathrm{H}$ & -3.94474293 & 3.37104877 & -0.00024618 \\
\hline $\mathrm{H}$ & -5.19315119 & 1.23005743 & -0.00008263 \\
\hline $\mathrm{I}$ & -5.19315053 & -1.23006053 & 0.00008224 \\
\hline I & -3.94474106 & -3.37105104 & 0.00024593 \\
\hline $\mathrm{H}$ & -1.49375082 & -3.39733981 & 0.00020600 \\
\hline $\mathrm{H}$ & 1.88397317 & -2.37652906 & -0.00035783 \\
\hline $\mathrm{H}$ & 0.53656638 & -3.11185551 & 0.88865683 \\
\hline $\mathrm{H}$ & 0.53615731 & -3.11210529 & -0.88848661 \\
\hline $\mathrm{H}$ & 1.88396767 & 2.37653728 & 0.00035796 \\
\hline $\mathrm{H}$ & 0.53655787 & 3.11185808 & -0.88865671 \\
\hline$H$ & 0.53614878 & 3.11210810 & 0.88848652 \\
\hline
\end{tabular}


$\mathrm{PhB}$ (mdan) $-\mathrm{F}^{-}$

Charge $=-1 ;$ Multiplicity $=1$

\begin{tabular}{|c|c|c|c|}
\hline $\mathrm{C}$ & 3.33422660 & 0.00000010 & 2.20736323 \\
\hline $\mathrm{C}$ & 4.61130867 & 0.00000024 & 1.63247735 \\
\hline $\mathrm{C}$ & 4.72910288 & 0.00000019 & 0.23964290 \\
\hline $\mathrm{C}$ & 3.58033188 & -0.00000000 & -0.56299215 \\
\hline $\mathrm{C}$ & 2.28442657 & -0.00000012 & -0.01506650 \\
\hline $\mathrm{C}$ & 2.19899008 & -0.00000009 & 1.39149999 \\
\hline B & 0.91659920 & -0.00000025 & -0.93798015 \\
\hline $\mathrm{N}$ & 0.06914739 & 1.25222425 & -0.67587345 \\
\hline $\mathrm{N}$ & 0.06914729 & -1.25222457 & -0.67587296 \\
\hline $\mathrm{C}$ & -1.22273407 & 1.25426488 & -0.22892866 \\
\hline C & -1.22273429 & -1.25426492 & -0.22892851 \\
\hline C & -1.93232135 & 2.45076904 & 0.00979937 \\
\hline C & -3.25842897 & 2.42604362 & 0.47558317 \\
\hline C & -3.91727510 & 1.23756963 & 0.71517652 \\
\hline 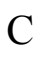 & -3.25044903 & 0.00000022 & 0.48629742 \\
\hline $\mathrm{C}$ & -1.89780229 & 0.00000006 & 0.01389587 \\
\hline C & -3.91727541 & -1.23756901 & 0.71517653 \\
\hline $\mathrm{C}$ & -3.25842956 & -2.42604316 & 0.47558316 \\
\hline$C$ & -1.93232194 & -2.45076891 & 0.00979943 \\
\hline F & 1.34797156 & -0.00000054 & -2.33319727 \\
\hline $\mathrm{C}$ & 0.70424815 & -2.52741879 & -0.94756935 \\
\hline C & 0.70424831 & 2.52741834 & -0.94757026 \\
\hline $\mathrm{H}$ & 3.22635643 & 0.00000013 & 3.29070337 \\
\hline Н & 5.49893438 & 0.00000039 & 2.26185955 \\
\hline $\mathrm{H}$ & 5.71556435 & 0.00000029 & -0.22183486 \\
\hline $\mathrm{H}$ & 3.68275561 & -0.00000007 & -1.64576304 \\
\hline $\mathrm{H}$ & 1.21445277 & -0.00000020 & 1.85650454 \\
\hline 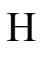 & -1.45751447 & 3.40828269 & -0.16150355 \\
\hline 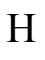 & -3.76841966 & 3.37263408 & 0.64879797 \\
\hline 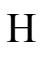 & -4.94361582 & 1.22537691 & 1.07390560 \\
\hline 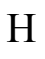 & -4.94361612 & -1.22537605 & 1.07390562 \\
\hline 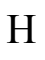 & -3.76842050 & -3.37263350 & 0.64879791 \\
\hline 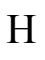 & -1.45751533 & -3.40828268 & -0.16150357 \\
\hline & 1.71081025 & -2.34786383 & -1.32628129 \\
\hline & 0.78249219 & -3.15546291 & -0.04512264 \\
\hline & 0.15448169 & -3.10256759 & -1.70879730 \\
\hline$\theta$ & 1.71081005 & 2.34786320 & -1.32628308 \\
\hline & 0.15448132 & 3.10256730 & -1.70879768 \\
\hline & 0.78249327 & 3.15546240 & -0.04512357 \\
\hline
\end{tabular}


$\mathrm{PhB}(\mathrm{aam})$

Charge $=0 ;$ Multiplicity $=1$

\begin{tabular}{|c|c|c|c|}
\hline C & -4.39183808 & 0.79416669 & 0.52773271 \\
\hline $\mathrm{C}$ & -4.96662303 & -0.38144636 & 0.03694847 \\
\hline $\mathrm{C}$ & -4.14694082 & -1.39093727 & -0.47556422 \\
\hline $\mathrm{C}$ & -2.76089225 & -1.22251946 & -0.49151183 \\
\hline $\mathrm{C}$ & -2.15578184 & -0.04674786 & -0.00462906 \\
\hline $\mathrm{C}$ & -3.00512142 & 0.95669447 & 0.50209211 \\
\hline 3 & -0.60080356 & 0.13719090 & -0.02323332 \\
\hline V & 0.29970632 & -0.96261278 & 0.09630920 \\
\hline $\mathrm{N}$ & 0.02106887 & 1.42396537 & -0.15849029 \\
\hline $\mathrm{C}$ & 1.68425628 & -0.81830336 & 0.08929422 \\
\hline$C$ & 1.39051953 & 1.67428548 & -0.18009464 \\
\hline$C$ & 2.53443776 & -1.93154143 & 0.21754861 \\
\hline$C$ & 3.91406027 & -1.76221620 & 0.20864799 \\
\hline $\mathrm{C}$ & 4.47797580 & -0.48401057 & 0.07174329 \\
\hline $\mathrm{C}$ & 3.64343842 & 0.61970124 & -0.05395354 \\
\hline $\mathrm{C}$ & 2.24771822 & 0.47124855 & -0.04551223 \\
\hline & 1.83130227 & 2.81384609 & -0.30793250 \\
\hline & -5.02231834 & 1.58137147 & 0.93136445 \\
\hline & -6.04523326 & -0.50945374 & 0.05234138 \\
\hline & -4.58711694 & -2.30419868 & -0.86602274 \\
\hline & -2.14390123 & -2.01353438 & -0.91268260 \\
\hline & -2.57894344 & 1.87406777 & 0.90186603 \\
\hline & -0.04235910 & -1.90150843 & 0.24702300 \\
\hline & -0.52209616 & 2.26934106 & -0.29063972 \\
\hline & 2.10367834 & -2.92430648 & 0.32251144 \\
\hline & 4.55761862 & -2.63164520 & 0.30835881 \\
\hline & 5.55605303 & -0.35994441 & 0.06500370 \\
\hline & 4.04153877 & 1.62337613 & -0.16068092 \\
\hline
\end{tabular}


$\mathrm{PhB}$ (aam)-F-

Charge $=-1$; Multiplicity $=1$

\begin{tabular}{|c|c|c|c|}
\hline$C$ & 3.54706468 & -0.47518200 & -1.76743028 \\
\hline $\mathrm{C}$ & 4.67442856 & -0.51042916 & -0.93768097 \\
\hline $\mathrm{C}$ & 4.51505329 & -0.30583884 & 0.43600313 \\
\hline $\mathrm{C}$ & 3.24076787 & -0.06950886 & 0.96849466 \\
\hline $\mathrm{C}$ & 2.09019315 & -0.03005569 & 0.16003288 \\
\hline $\mathrm{C}$ & 2.28228200 & -0.23943151 & -1.21975801 \\
\hline B & 0.59635220 & 0.23745370 & 0.77681475 \\
\hline $\mathrm{N}$ & -0.34606862 & -0.93912656 & 0.50470278 \\
\hline$N$ & -0.06681002 & 1.47533587 & 0.15259087 \\
\hline $\mathrm{C}$ & -1.66705117 & -0.82811100 & 0.19781157 \\
\hline $\mathrm{C}$ & -1.36399523 & 1.65922391 & -0.19772857 \\
\hline $\mathrm{C}$ & -2.53493920 & -1.95343177 & 0.18776002 \\
\hline $\mathrm{C}$ & -3.87678587 & -1.82401168 & -0.14695271 \\
\hline $\mathrm{C}$ & -4.41706431 & -0.57282922 & -0.48841396 \\
\hline $\mathrm{C}$ & -3.57501821 & 0.53908647 & -0.48832595 \\
\hline $\mathrm{C}$ & -2.22055225 & 0.43752735 & -0.15419399 \\
\hline $\mathrm{O}$ & -1.82084668 & 2.76333600 & -0.55980536 \\
\hline F & 0.74488450 & 0.43031822 & 2.21558482 \\
\hline $\mathrm{H}$ & 3.65653296 & -0.63060483 & -2.83940454 \\
\hline H & 5.66157891 & -0.69310047 & -1.35751587 \\
\hline $\mathrm{H}$ & 5.38342218 & -0.32903523 & 1.09262430 \\
\hline $\mathrm{H}$ & 3.12406093 & 0.09121574 & 2.03718067 \\
\hline $\mathrm{H}$ & 1.41511418 & -0.21369138 & -1.87699308 \\
\hline $\mathrm{H}$ & -0.04412818 & -1.86505049 & 0.77619964 \\
\hline $\mathrm{H}$ & 0.45863742 & 2.34118768 & 0.11078932 \\
\hline $\mathrm{H}$ & -2.12873617 & -2.92830754 & 0.45236319 \\
\hline $\mathrm{H}$ & -4.51270470 & -2.70764904 & -0.14040421 \\
\hline & -5.46754263 & -0.47445883 & -0.74812180 \\
\hline$H$ & -3.94133220 & 1.52716061 & -0.75137432 \\
\hline
\end{tabular}


$\mathrm{PhB}$ (pin)

Charge $=0 ;$ Multiplicity $=1$

$\begin{array}{lrrc}\mathrm{C} & -3.80163956 & -1.20004216 & 0.16061624 \\ \mathrm{C} & -4.50172929 & 0.00000063 & 0.00000697 \\ \mathrm{C} & -3.80163956 & 1.20004244 & -0.16060987 \\ \mathrm{C} & -2.40537184 & 1.19715659 & -0.15924658 \\ \mathrm{C} & -1.68199862 & -0.00000142 & -0.00000834 \\ \mathrm{C} & -2.40537183 & -1.19715816 & 0.15923928 \\ \mathrm{~B} & -0.12650052 & -0.00000337 & -0.00002107 \\ \mathrm{O} & 0.63597518 & 1.12711248 & -0.20772865 \\ \mathrm{C} & 2.02404709 & 0.78600463 & 0.08308597 \\ \mathrm{C} & 2.02404920 & -0.78600385 & -0.08308317 \\ \mathrm{O} & 0.63597593 & -1.12711202 & 0.20772190 \\ \mathrm{C} & 2.29993718 & -1.24988555 & -1.51993811 \\ \mathrm{C} & 2.92277611 & -1.54194531 & 0.89340305 \\ \mathrm{C} & 2.29992708 & 1.24988412 & 1.51994339 \\ \mathrm{C} & 2.92277916 & 1.54194901 & -0.89339290 \\ \mathrm{H} & -4.34387374 & -2.13334972 & 0.28555043 \\ \mathrm{H} & -5.58845217 & 0.00000140 & 0.00001269 \\ \mathrm{H} & -4.34387375 & 2.13335064 & -0.28553916 \\ \mathrm{H} & -1.86350170 & 2.13080940 & -0.28308569 \\ \mathrm{H} & -1.86350168 & -2.13081194 & 0.28307093 \\ \mathrm{H} & 1.65847324 & -0.73206566 & -2.23879680 \\ \mathrm{H} & 3.34426703 & -1.08412460 & -1.80235847 \\ \mathrm{H} & 2.64674836 & 1.34666527 & -1.93122837 \\ \mathrm{H} & 2.09028650 & -2.32101180 & -1.59067746 \\ \mathrm{H} & 2.83040442 & -2.61757375 & 0.71637062 \\ \mathrm{H} & 3.97259562 & -1.26350978 & 0.75051938 \\ \mathrm{H} & 2.64673813 & -1.34665987 & 1.93123632 \\ \mathrm{H} & 1.65846028 & 0.73206157 & 2.23879764 \\ \mathrm{H} & 3.34425566 & 1.08412499 & 1.80236976 \\ \mathrm{H} & 2.09027372 & 2.32100975 & 1.59068347 \\ \mathrm{H} & 3.97259796 & 1.26351436 & -0.75050260 \\ \mathrm{H} & 2.61757702 & -0.71635894\end{array}$


$\mathrm{PhB}$ (pin)-F-

Charge $=-1 ;$ Multiplicity $=1$

$\begin{array}{lccc}\mathrm{C} & -3.39179243 & 1.43456802 & -0.37689251 \\ \mathrm{C} & -4.30543480 & 0.55357240 & 0.21033121 \\ \mathrm{C} & -3.87052260 & -0.72356491 & 0.58678353 \\ \mathrm{C} & -2.54131572 & -1.10318227 & 0.37485197 \\ \mathrm{C} & -1.59577287 & -0.23475373 & -0.20617659 \\ \mathrm{C} & -2.06220415 & 1.03965112 & -0.57589185 \\ \mathrm{~B} & -0.04324230 & -0.70189132 & -0.41474135 \\ \mathrm{O} & 0.69859797 & -0.88531358 & 0.86383307 \\ \mathrm{C} & 1.77202465 & 0.03376136 & 0.93066675 \\ \mathrm{C} & 2.04333232 & 0.36897672 & -0.59025668 \\ \mathrm{O} & 0.74036569 & 0.31658640 & -1.14709559 \\ \mathrm{C} & 2.63934431 & 1.75955760 & -0.84910751 \\ \mathrm{C} & 2.93253456 & -0.69296282 & -1.27578064 \\ \mathrm{C} & 2.95146610 & -0.62327309 & 1.66364618 \\ \mathrm{C} & 1.31679324 & 1.27504269 & 1.73107914 \\ \mathrm{~F} & -0.05614566 & -1.97395188 & -1.10962315 \\ \mathrm{H} & -3.71593928 & 2.42934425 & -0.68086310 \\ \mathrm{H} & -5.33945977 & 0.85418227 & 0.36988617 \\ \mathrm{H} & -4.57125244 & -1.42229491 & 1.04240867 \\ \mathrm{H} & -2.22196037 & -2.10241424 & 0.66399727 \\ \mathrm{H} & -1.35832584 & 1.72982389 & -1.03582892 \\ \mathrm{H} & 1.97031934 & 2.54834974 & -0.49586207 \\ \mathrm{H} & 3.61431400 & 1.87690915 & -0.35765445 \\ \mathrm{H} & 2.78271952 & 1.89953739 & -1.92703732 \\ \mathrm{H} & 2.91041815 & -0.51355086 & -2.35652549 \\ \mathrm{H} & 3.97600495 & -0.64507198 & -0.93702777 \\ \mathrm{H} & 2.53766522 & -1.69568728 & -1.09601660 \\ \mathrm{H} & 3.21699080 & -1.57711537 & 1.20163911 \\ \mathrm{H} & 3.83633926 & 0.02758780 & 1.67055755 \\ \mathrm{H} & -0.82293509 & 2.70364495 \\ \mathrm{H} & -1.96745907 & -89286726 & 1.89602257 \\ \mathrm{H} & -1.78225449 & 1.21919721 \\ \mathrm{H} & 0.93869591 & 2.70635936\end{array}$




\section{Computational Details $\left(\% V_{\text {bur }}\right)^{13,14}$}

Buried volumes for NHC-containing $\mathrm{CuCl}$ complexes were calculated based on crystallographic data (CCDC deposition numbers were listed in the table) using SambVca 2.1 Web tool. When several molecules were found existing in the unit cell, the average buried volume was calculated. We use the following parameters: bond radii scaled by 1.17 ; sphere radius $=3.5$; distance of the coordination point from the center of the sphere $=0.0 ;$ mesh spacing for numerical integration $=0.10 ; \mathrm{H}$ atoms were omitted for calculations.

Table S3. Calculation of $\% V_{\text {bur }}$

\begin{tabular}{|c|c|c|}
\hline $\mathrm{NHCCuCl}$ & $\% V_{\text {bur }}$ & $\begin{array}{c}\text { Reference } \\
\text { Deposition Number }\end{array}$ \\
\hline \hline $\mathrm{IMesCCl}$ & 38.1 & 979584 \\
\hline $\mathrm{IPrCuCl}$ & 48.5 & 234224 \\
\hline $\mathrm{SIPrCuCl}$ & 50.1 & 1861502 \\
\hline${ }^{6 \mathrm{DippCuCl}}$ & 53.4 & 2085995 \\
\hline${ }^{\mathrm{Cl}} \mathrm{IMesCuCl}$ & 37.9 & 2120464 \\
\hline${ }^{\mathrm{Cl}} \mathrm{IPrCuCl}$ & 47.1 & 2085996 \\
\hline
\end{tabular}




\section{Mechanistic Studies on a Catalytic Cycle}

Scheme S1. Hydroboration in the Presence of MeOD

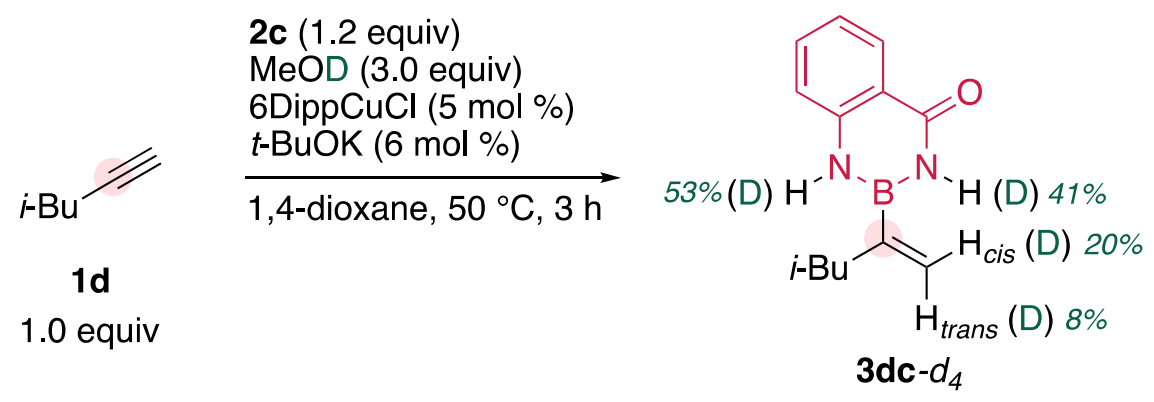

After the reaction was completed, the solvent was removed in vacuo. The deuterium-incorporation ratio was determined by ${ }^{1} \mathrm{H}$ NMR spectrum of the crude product solution in DMSO- $d_{6}$.

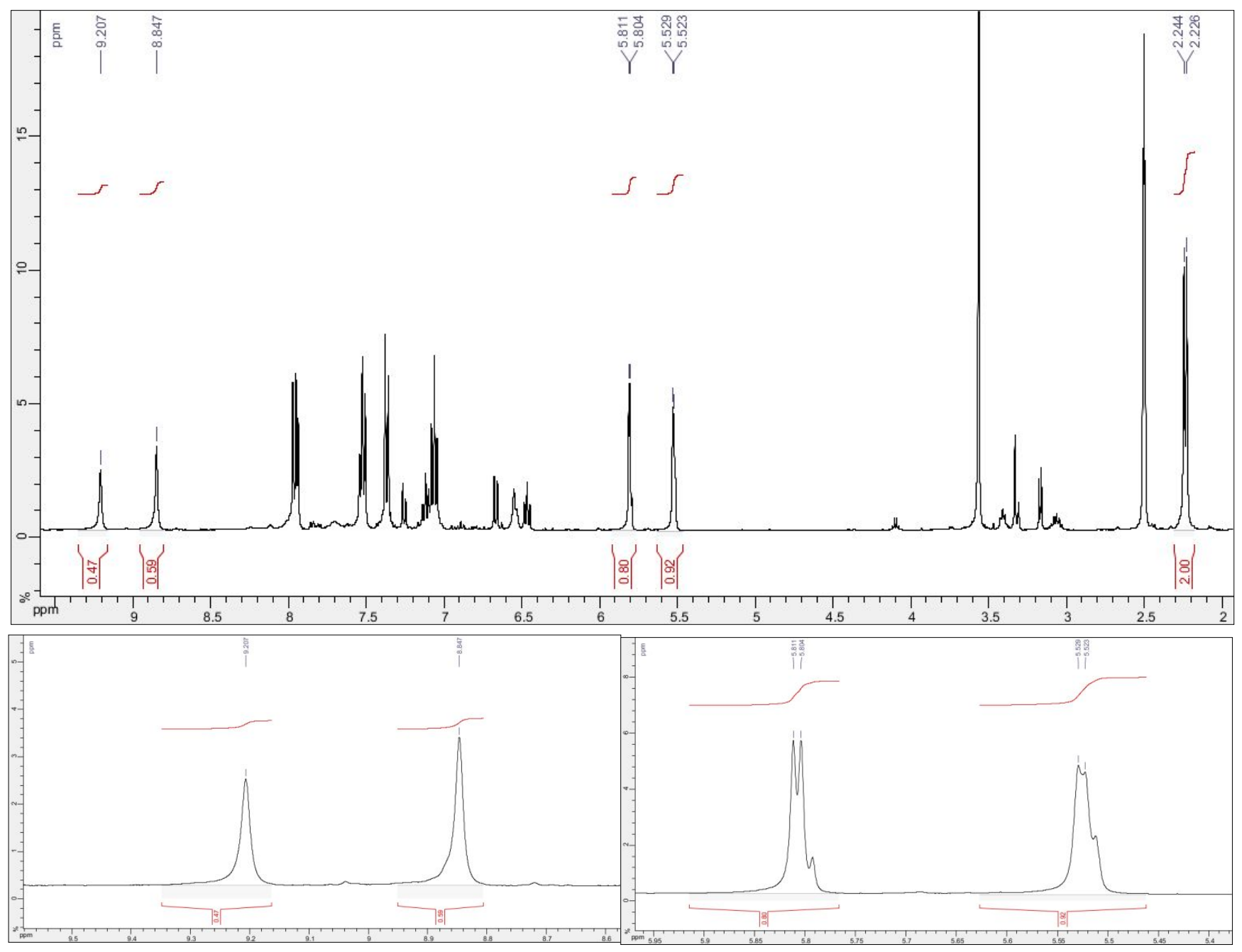


Scheme S2. Treatment of 3dc with MeOD

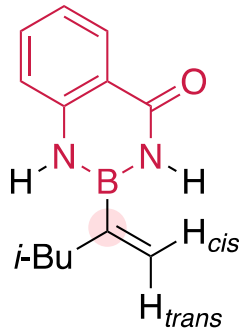

3dc

1.0 equiv

(n)

\section{MeOD (3.0 equiv)}

$6 \mathrm{DippCuCl}(5 \mathrm{~mol} \%)$

$t$-BuOK $(6 \mathrm{~mol} \%)$

1,4-dioxane, $50^{\circ} \mathrm{C}, 3 \mathrm{~h}$

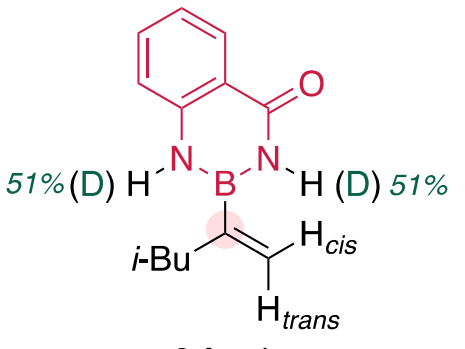

3 dc $-d_{2}$

The deuterium-incorporation ratio was determined by ${ }^{1} \mathrm{H}$ NMR spectrum of the crude product solution in DMSO- $d_{6}$.
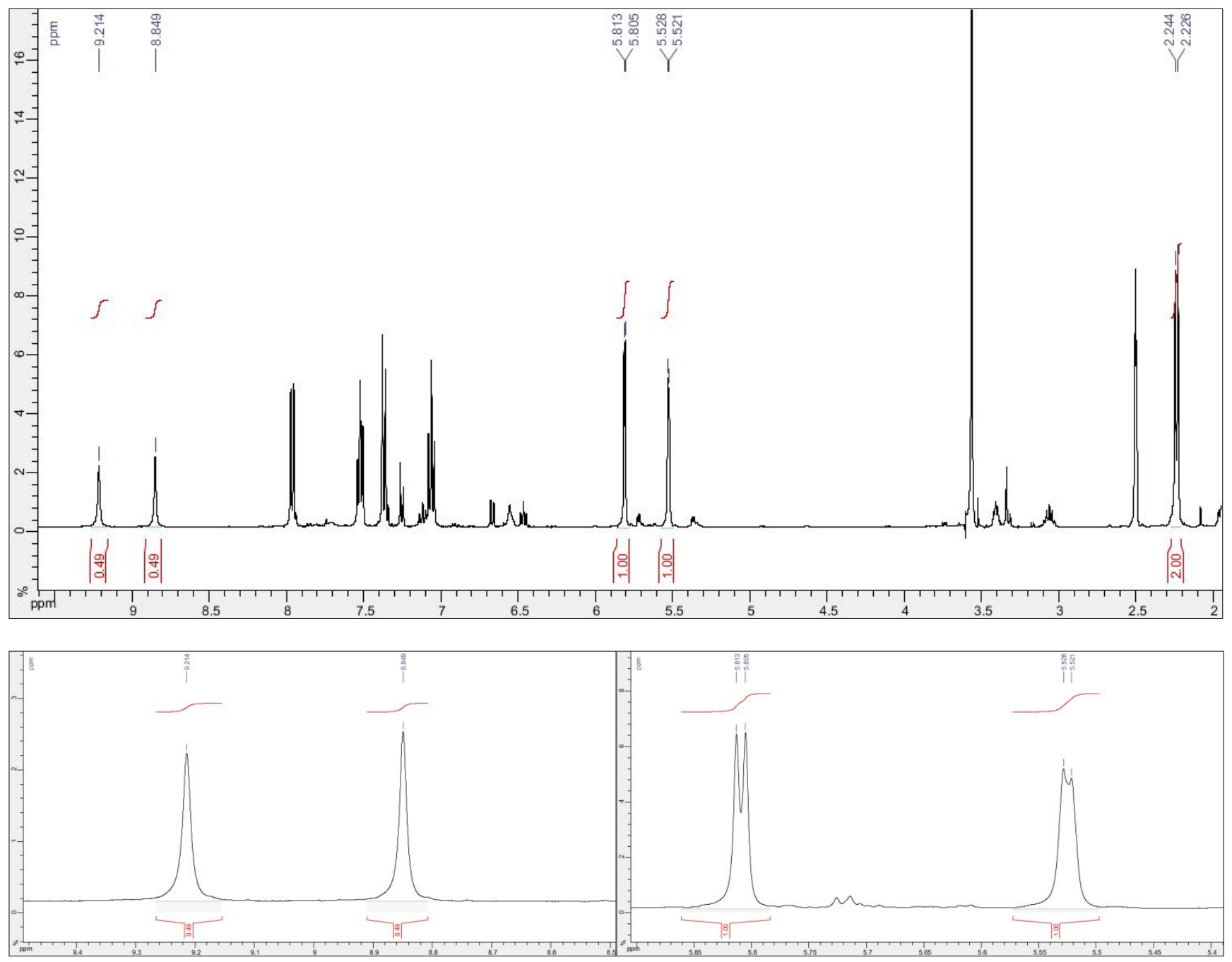
Scheme S3. Treatment of 1c with MeOD

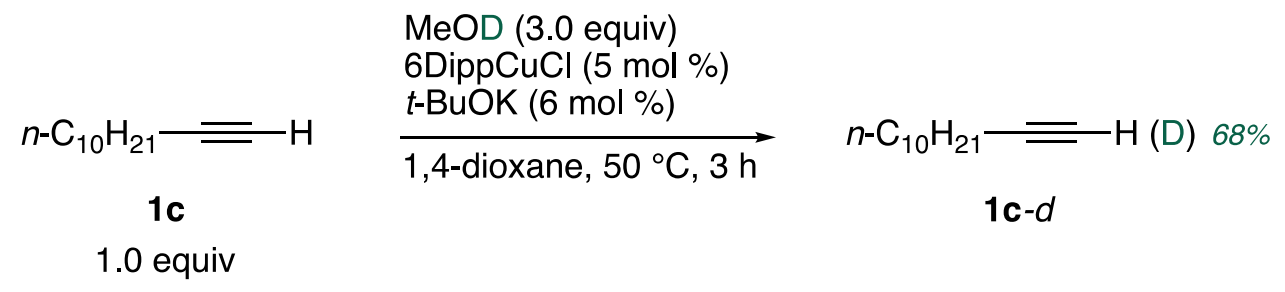

The deuterium-incorporation ratio was determined by ${ }^{1} \mathrm{H}$ NMR spectrum of the crude product solution in $\mathrm{CDCl}_{3}$.

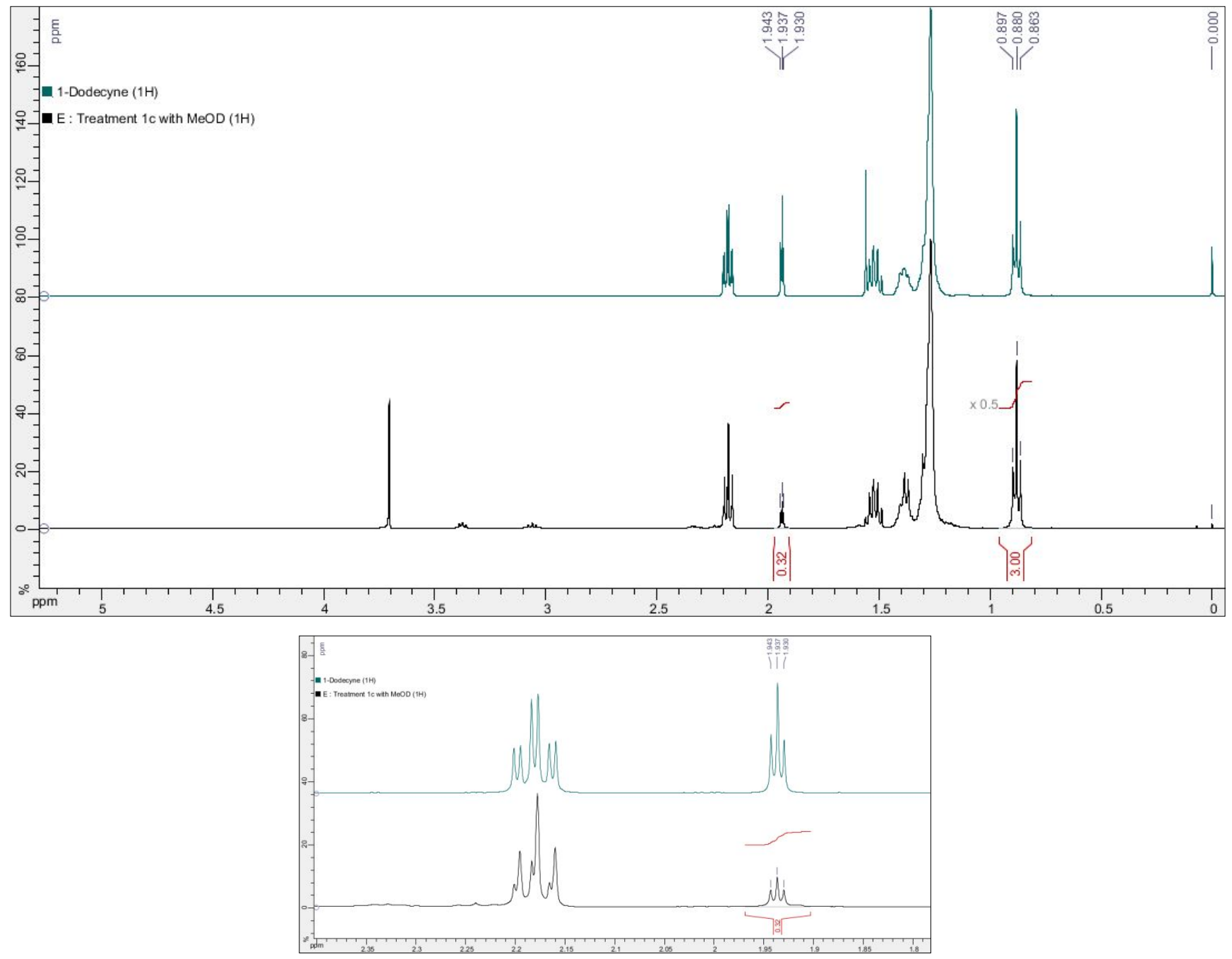


Figure S1. ${ }^{11} \mathrm{~B}$ NMR Experiments Determining a $\mathrm{Cu}-\mathrm{B}$ (aam) species and a $\mathrm{Cu}-\mathrm{B}$ (pin) species

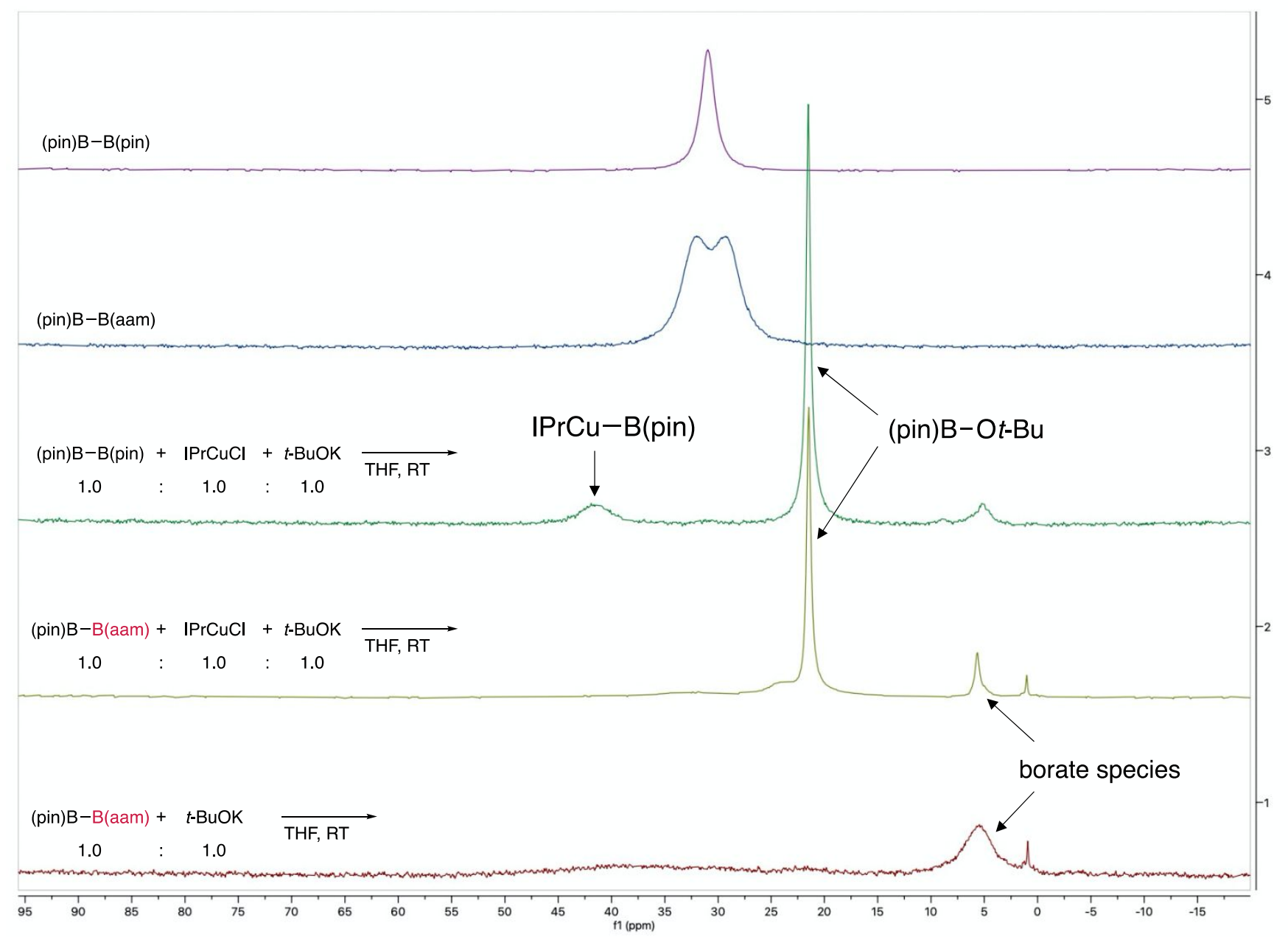

Stoichiometric treatment of (pin)B-B(aam) with $\mathrm{IPrCuCl}$ and $t$-BuOK immediately generated (pin)B-O $t$ $\mathrm{Bu}$ with complete consumption of the diboron (see lines 2 and 4), however $\mathrm{IPrCu}-\mathrm{B}(\mathrm{aam})$, whose ${ }^{11} \mathrm{~B}$ NMR signal is supposed to appear at $c a .40 \mathrm{ppm},{ }^{15,16}$ could not be detected. In contrast, a similar treatment of (pin)B-B(pin) led to clear observation of $\mathrm{IPrCu}-\mathrm{B}(\mathrm{pin})^{15}$ and (pin)B-Ot-Bu (see lines 3 and 5). A minor signal at upfield region would arise from some borate species (see lines 1 and 2). 
Figure S2. Variable Temperature ${ }^{11} \mathrm{~B}$ NMR Experiments Determining a $\mathrm{Cu}-\mathrm{B}(\mathrm{aam})$ species

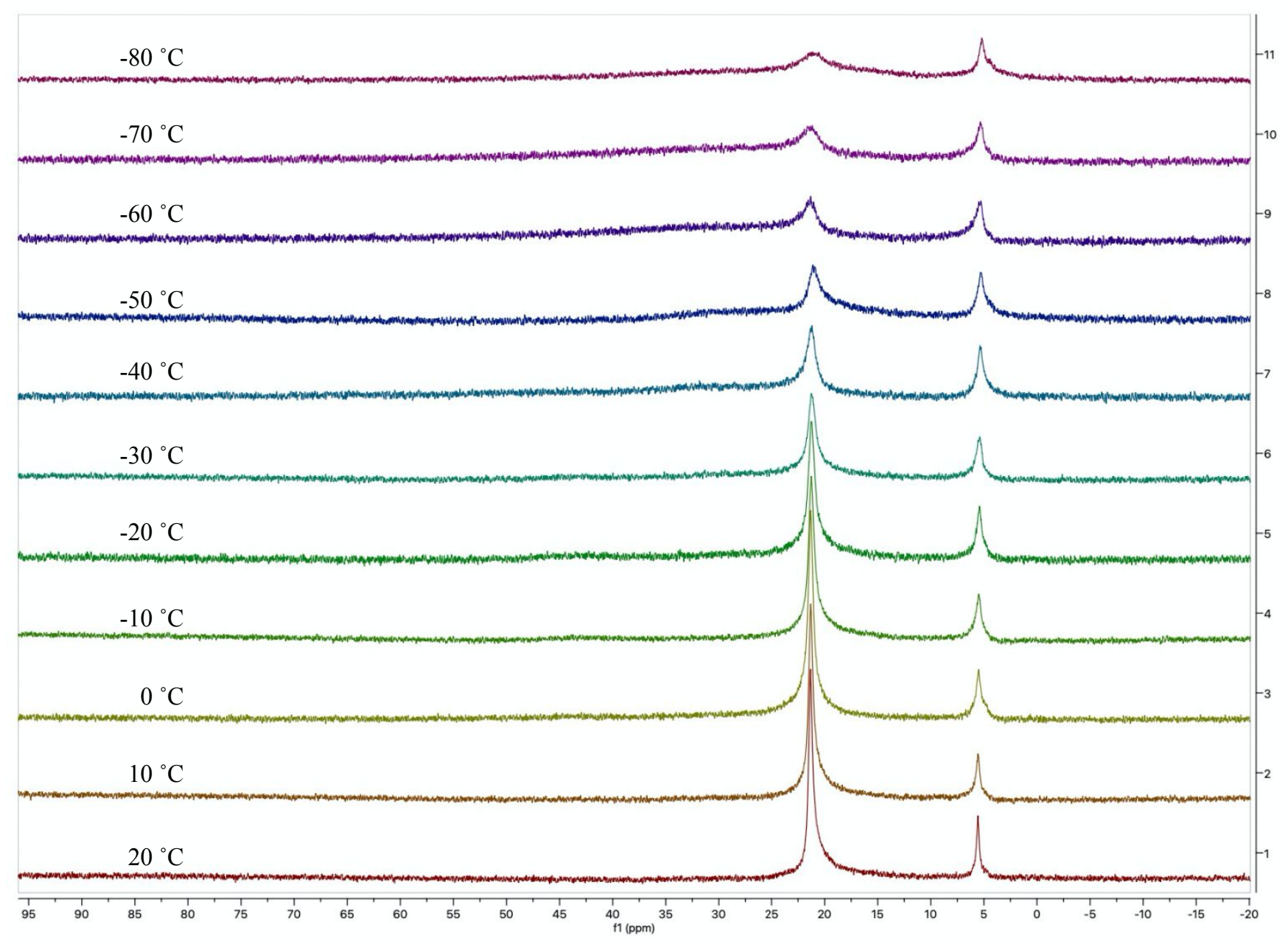

The treatment of (pin)B-B(aam) with $\mathrm{IPrCuCl}$ and $t$-BuOK even at $-80^{\circ} \mathrm{C}$ immediately generated (pin)B$\mathrm{O} t$ - $\mathrm{Bu}(21.5 \mathrm{ppm})$ with the diboron (29.1 and $32.1 \mathrm{ppm})$ disappearing; the result strongly implied the coproduction of $\mathrm{IPrCu}-\mathrm{B}(\mathrm{aam})$. However, this borylcopper species could not be detected at any temperature, probably owing to its extreme instability under alkyne-free conditions. 


\section{Experimental Procedures and Characterization of Products}

\section{Synthesis of butyl hex-5-ynoate (1j). ${ }^{17}$}<smiles>C#CCCCC(=O)OCCCC</smiles>

To a solution of 5-hexynoic acid $(1.8 \mathrm{mmol}, 203 \mathrm{mg})$ in DCM $(10 \mathrm{~mL})$ were added $n$-BuOH (4 equiv., $7.2 \mathrm{mmol}, 0.53 \mathrm{~g}, 0.66 \mathrm{~mL}$ ), DMAP (0.1 equiv., $0.18 \mathrm{mmol}, 38 \mathrm{mg}$ ) and DCC (1.5 equiv., $2.7 \mathrm{mmol}, 0.56$ g). After stirring for $2 \mathrm{~h}$, the obtained white suspension was filtrated and concentrated. The residue was purified by flash chromatography to afford $\mathbf{1 j}$ (222 $\mathrm{mg}, 1.3 \mathrm{mmol}, 73 \%$ ) as a colorless oil.

${ }^{1} \mathrm{H}$ NMR $\left(\mathrm{CDCl}_{3}\right) \delta 0.91(\mathrm{t}, J=7.4 \mathrm{~Hz}, 3 \mathrm{H}), 1.29-1.43(\mathrm{~m}, 2 \mathrm{H}), 1.53-1.65(\mathrm{~m}, 2 \mathrm{H}), 1.77-1.89(\mathrm{~m}$, 2H), $1.95(\mathrm{t}, J=2.7 \mathrm{~Hz}, 1 \mathrm{H}), 2.25(\mathrm{td}, J=7.0,2.6 \mathrm{~Hz}, 2 \mathrm{H}), 2.43(\mathrm{t}, J=7.4 \mathrm{~Hz}, 2 \mathrm{H}), 4.06(\mathrm{t}, J=6.7 \mathrm{~Hz}$, 2H)

${ }^{13} \mathrm{C} \mathrm{NMR}\left(\mathrm{CDCl}_{3}\right) \delta 13.69,17.84,19.11,23.63,30.63,32.93,64.29,69.03,83.28,173.14$

\section{Synthesis of tert-butyl(hex-5-yn-1-yloxy)dimethylsilane (1k). ${ }^{18}$}<smiles>C#CCCCCO[SbH3]</smiles>

Hex-5-yn-1-ol (5.0 mmol, $490 \mathrm{mg})$ was dissolved in dry DCM (12 mL), and was treated with $\mathrm{Et}_{3} \mathrm{~N}(10$ mmol, $1.01 \mathrm{~g})$ and TBSCl $(6.0 \mathrm{mmol}, 0.91 \mathrm{~g})$ at room temperature for $6 \mathrm{~h}$. Then $\mathrm{H}_{2} \mathrm{O}(30 \mathrm{~mL})$ were added to the reaction mixture, and the aqueous layer was further extracted with DCM. The combined organic solution was washed with brine, dried over $\mathrm{Na}_{2} \mathrm{SO}_{4}$, and concentrated under reduced pressure. Purification by flash chromatography (20:1 hexane/ethyl acetate as an eluent) provided 1k (433 mg, $41 \%$ ) as a colorless oil.

${ }^{1} \mathrm{H}$ NMR $\left(\mathrm{CDCl}_{3}\right) \delta 0.04(\mathrm{~s}, 6 \mathrm{H}), 0.88(\mathrm{~d}, J=0.5 \mathrm{~Hz}, 9 \mathrm{H}), 1.58-1.67(\mathrm{~m}, 4 \mathrm{H}), 1.93(\mathrm{t}, J=2.7 \mathrm{~Hz}, 1 \mathrm{H})$, $2.16-2.25(\mathrm{~m}, 2 \mathrm{H}), 3.62(\mathrm{t}, J=6.0 \mathrm{~Hz}, 2 \mathrm{H})$

${ }^{13} \mathrm{C} \mathrm{NMR}\left(\mathrm{CDCl}_{3}\right) \delta-5.33,18.19,18.31,24.93,25.93,31.78,62.55,68.24,84.47$

\section{Synthesis of 5-ethynyl-1,2,3-trimethoxybenzene (1q). ${ }^{19}$}

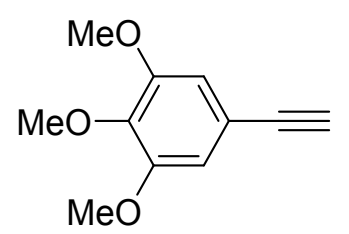

3,4,5-Trimethoxybromobenzene (3.0 g, $12.15 \mathrm{mmol}), \mathrm{Pd}\left(\mathrm{PPh}_{3}\right)_{4}(716 \mathrm{mg}, 0.68 \mathrm{mmol})$, CuI (116 mg, 0.68 $\mathrm{mmol}), \mathrm{PPh}_{3}(179 \mathrm{mg}, 0.73 \mathrm{mmol})$ were taken in an oven dried round bottom flask and placed under vacuum for $30 \mathrm{~min}$. TMS-acetylene (1.8 g, $18.2 \mathrm{mmol})$ and triethylamine $(15 \mathrm{~mL})$ were added via syringe and the reaction was carried out at $90{ }^{\circ} \mathrm{C}$ for $18 \mathrm{~h}$. The TMS-protected product was purified using column 
chromatography with (7:1 hexane/ethyl acetate as an eluent) to yield the product $(2.7 \mathrm{~g}, 8.5 \mathrm{mmol})$ as an orange-brown oil. This product was dissolved in DCM-Methanol (1:4) solvent mixture (25 mL), and $\mathrm{K}_{2} \mathrm{CO}_{3}(1.3 \mathrm{~g}, 9.1 \mathrm{mmol})$ was added. After stirring for $5 \mathrm{~h}, \mathbf{1 q}$ was obtained $(2.0 \mathrm{~g}, 8.5 \mathrm{mmol}, 86 \%$ overall) as pale yellow solid.

${ }^{1} \mathrm{H}$ NMR $\left(\mathrm{CDCl}_{3}\right) \delta 3.03(\mathrm{~s}, 1 \mathrm{H}), 3.85(\mathrm{~s}, 9 \mathrm{H}), 6.73(\mathrm{~s}, 2 \mathrm{H})$

${ }^{13} \mathrm{C} \mathrm{NMR}\left(\mathrm{CDCl}_{3}\right) \delta 56.13,60.95,76.20,83.69,109.30,117.00,153.02$

\section{Synthesis of (pin)B-B(mdan) (2d).}

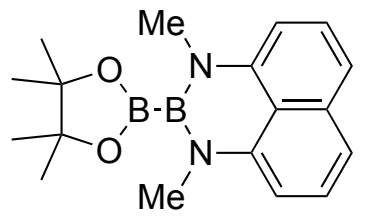

A flame-dried two-necked round-bottomed flask equipped with a magnetic stirring bar was charged with (pin)B-B(dan) $(5.0 \mathrm{mmol})$, THF $(5.0 \mathrm{~mL})$ before the mixture was stirred at $60{ }^{\circ} \mathrm{C}$. Iodomethane $(10$ mmol) was added, and then lithium bis(trimethylsilyl)amide (1.0 M solution in THF, $10 \mathrm{mmol}$ ) was dropped for $10 \mathrm{~min}$. And again, iodomethane $(10 \mathrm{mmol})$ was added to the reaction mixture, and then lithium bis(trimethylsilyl)amide (1.0 M solution in THF, $10 \mathrm{mmol})$ was dropped for $10 \mathrm{~min}$. The mixture was diluted with ethyl acetate and saturated $\mathrm{NH}_{4} \mathrm{Cl}$ aq $(20 \mathrm{~mL})$, and the organic solution was washed with brine $(20 \mathrm{~mL} \times 3)$, dried over $\mathrm{Na}_{2} \mathrm{SO}_{4}$, and evaporated. Purification of the residue by silica gel-column chromatography (hexane/ethyl acetate $=10: 1$ as an eluent), followed by recrystallization in isopropyl alcohol $\left(70{ }^{\circ} \mathrm{C}\right.$ to $\left.\mathrm{rt}\right)$ gave $\mathbf{2 d}$ as a white solid $(0.81 \mathrm{~g}, 50 \%)$.

A white solid: $\mathrm{mp} 115-117^{\circ} \mathrm{C}$

${ }^{1} \mathrm{H} \mathrm{NMR}\left(\mathrm{CDCl}_{3}\right) \delta 1.36(\mathrm{~s}, 12 \mathrm{H}), 3.14(\mathrm{~s}, 6 \mathrm{H}), 6.41(\mathrm{~d}, J=7.7 \mathrm{~Hz}, 2 \mathrm{H}), 7.13(\mathrm{~d}, J=8.2 \mathrm{~Hz}, 2 \mathrm{H}), 7.25$ (t, $J=7.9 \mathrm{~Hz}, 2 \mathrm{H})$.

${ }^{13} \mathrm{C} \mathrm{NMR}\left(\mathrm{CDCl}_{3}\right) \delta 24.96,37.37,83.14,102.84,117.85,120.93,127.11,135.81,142.63$.

${ }^{11} \mathrm{~B} \mathrm{NMR}\left(\mathrm{CDCl}_{3}\right) \delta 32.44$

HRMS Calcd for $\mathrm{C}_{18} \mathrm{H}_{25} \mathrm{O}_{2} \mathrm{~B}_{2} \mathrm{~N}_{2}:[\mathrm{M}+\mathrm{H}]^{+}$, 323.2097. Found: $m / z 323.2099$

\section{Cu-Catalyzed Hydroboration of Alkynes.}

A Schlenk tube equipped with a magnetic stirring bar was charged with $6 \mathrm{DippCuCl}(7.5 \mu \mathrm{mol}), t-\mathrm{BuOK}$ $(1.0 \mathrm{M}$ solution in THF, $9.0 \mu \mathrm{mol})$ and 1,4-dioxane $(0.5 \mathrm{~mL})$ before the mixture was stirred at room temperature for $10 \mathrm{~min}$. To the mixture was added (pin)B-B(aam) $(0.18 \mathrm{mmol}), \mathrm{MeOH}(0.45 \mathrm{mmol})$ and an alkyne $(0.15 \mathrm{mmol})$, and the resulting mixture was stirred at $50{ }^{\circ} \mathrm{C}$ for $3 \mathrm{~h}$. The mixture was diluted with ethyl acetate and filtered through a Celite plug. The organic solution was washed with brine, dried over $\mathrm{Na}_{2} \mathrm{SO}_{4}$, and evaporated. Purification of the residue by boric acid impregnated silica gel-column chromatography ${ }^{14}$ (hexane/ethyl acetate as an eluent) or gel permeation chromatography (toluene as an 
eluent) gave the product.

2-(oct-1-en-2-yl)-2,3-dihydrobenzo[d] $[1,3,2]$ diazaborinin-4(1H)-one (3ac)

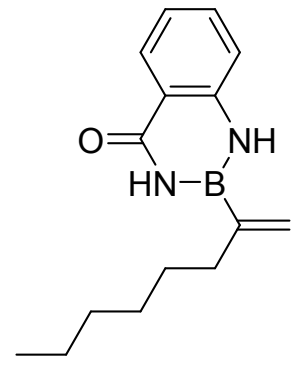

A pale yellow solid: $\mathrm{mp} 93-95^{\circ} \mathrm{C}$

${ }^{1} \mathrm{H}$ NMR $\left(\mathrm{CDCl}_{3}\right) \delta 0.88(\mathrm{t}, J=7.0 \mathrm{~Hz}, 3 \mathrm{H}), 1.26-1.36(\mathrm{~m}, 6 \mathrm{H}), 1.41-1.48(\mathrm{~m}, 2 \mathrm{H}), 2.26(\mathrm{t}, J=7.8 \mathrm{~Hz}$, 2H), 5.64 (s, 2H), 6.58 (brs, 1H), 7.05 (d, $J=8.2 \mathrm{~Hz}, 1 \mathrm{H}), 7.13$ (ddd, $J=8.1,7.2,1.1 \mathrm{~Hz}, 1 \mathrm{H}), 7.36$ (brs, $1 \mathrm{H}), 7.51$ (ddd, $J=8.5,7.3,1.6 \mathrm{~Hz}, 1 \mathrm{H}), 8.21(\mathrm{dd}, J=8.0,1.5 \mathrm{~Hz}, 1 \mathrm{H})$

${ }^{13} \mathrm{C} \mathrm{NMR}\left(\mathrm{CDCl}_{3}\right) \delta 14.07,22.61,29.07,29.33,31.68,35.27,117.47,118.92,121.73,125.57,129.11$, $133.76,144.11,166.51$

${ }^{11} \mathrm{~B} \mathrm{NMR}\left(\mathrm{CDCl}_{3}\right) \delta 28.64$

HRMS Calcd for $\mathrm{C}_{15} \mathrm{H}_{21} \mathrm{BN}_{2} \mathrm{ONa}$ : [M+Na] $]^{+}$279.1639. Found: $m / z 279.1641$

\section{2-(hex-1-en-2-yl)-2,3-dihydrobenzo[d][1,3,2] diazaborinin-4(1H)-one (3bc)}

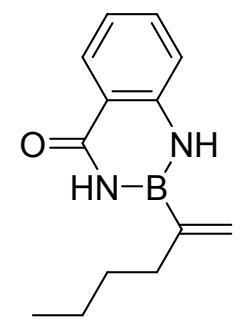

A pale yellow solid: $\mathrm{mp} 83-86{ }^{\circ} \mathrm{C}$

${ }^{1} \mathrm{H} \mathrm{NMR}\left(\mathrm{CDCl}_{3}\right) \delta 0.92(\mathrm{t}, J=7.2 \mathrm{~Hz}, 3 \mathrm{H}), 1.30-1.39(\mathrm{~m}, 2 \mathrm{H}), 1.40-1.49(\mathrm{~m}, 2 \mathrm{H}), 2.27(\mathrm{t}, J=7.8 \mathrm{~Hz}$, 2H), $5.64(\mathrm{~s}, 1 \mathrm{H}), 5.65(\mathrm{~s}, 1 \mathrm{H}), 6.55$ (brs, $1 \mathrm{H}), 7.05$ (dd, $J=8.2,1.0 \mathrm{~Hz}, 1 \mathrm{H}), 7.14$ (ddd, $J=8.1,7.2,1.1$ $\mathrm{Hz}, 1 \mathrm{H}), 7.31$ (brs, $1 \mathrm{H}), 7.52(\mathrm{ddd}, J=8.1,7.2,1.6 \mathrm{~Hz}, 1 \mathrm{H}), 8.21(\mathrm{dd}, J=7.9,1.7 \mathrm{~Hz}, 1 \mathrm{H})$ ${ }^{13} \mathrm{C} \mathrm{NMR}\left(\mathrm{CDCl}_{3}\right) \delta 13.95,22.46,31.52,34.97,117.48,118.91,121.72,125.59,129.09,133.76,144.12$, 166.54

${ }^{11} \mathrm{~B}$ NMR $\left(\mathrm{CDCl}_{3}\right) \delta 28.80$

HRMS Calcd for $\mathrm{C}_{13} \mathrm{H}_{17} \mathrm{BN}_{2} \mathrm{ONa}$ : $[\mathrm{M}+\mathrm{Na}]^{+}, 251.1326$. Found: $m / z 251.1327$

2-(dodec-1-en-2-yl)-2,3-dihydrobenzo[d][1,3,2] diazaborinin-4(1H)-one (3cc) 


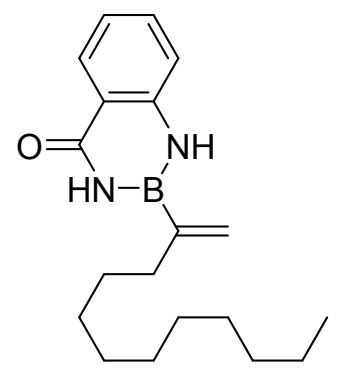

A pale yellow solid: $\mathrm{mp} 76-77^{\circ} \mathrm{C}$

${ }^{1} \mathrm{H}$ NMR $\left(\mathrm{CDCl}_{3}\right) \delta 0.87(\mathrm{t}, J=7.0 \mathrm{~Hz}, 3 \mathrm{H}), 1.20-1.35(\mathrm{~m}, 14 \mathrm{H}), 1.40-1.50(\mathrm{~m}, 2 \mathrm{H}), 2.26(\mathrm{t}, J=7.6$ $\mathrm{Hz}, 2 \mathrm{H}), 5.64$ (s, 2H), 6.57 (brs, 1H), 7.05 (d, $J=8.1 \mathrm{~Hz}, 1 \mathrm{H}), 7.13$ (ddd, $J=8.0,7.2,1.0 \mathrm{~Hz}, 1 \mathrm{H}), 7.35$ (brs, $1 \mathrm{H}), 7.51(\mathrm{ddd}, J=8.5,7.2,1.6 \mathrm{~Hz}, 1 \mathrm{H}), 8.21(\mathrm{dd}, J=7.9,1.6 \mathrm{~Hz}, 1 \mathrm{H})$

${ }^{13} \mathrm{C} \mathrm{NMR}\left(\mathrm{CDCl}_{3}\right) \delta 14.10,22.66,29.30,29.36,29.40,29.48,29.59,31.87,35.26,117.48,118.90,121.70$, $125.58,129.09,133.75,144.14,166.59$

${ }^{11} \mathrm{~B}$ NMR $\left(\mathrm{CDCl}_{3}\right) \delta 28.74$

HRMS Calcd for $\mathrm{C}_{19} \mathrm{H}_{29} \mathrm{BN}_{2} \mathrm{ONa}:[\mathrm{M}+\mathrm{Na}]^{+}, 335.2265$. Found: $m / z 335.2268$

\section{2-(4-methylpent-1-en-2-yl)-2,3-dihydrobenzo $[d][1,3,2]$ diazaborinin-4(1H)-one (3dc)}

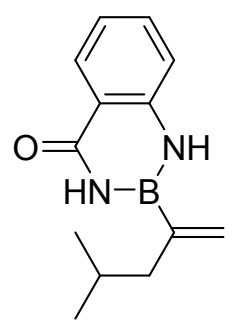

A pale yellow solid: $\mathrm{mp} 109-110{ }^{\circ} \mathrm{C}$

${ }^{1} \mathrm{H}$ NMR $\left(\mathrm{CDCl}_{3}\right) \delta 0.91(\mathrm{~d}, J=6.6 \mathrm{~Hz}, 6 \mathrm{H}), 1.66-1.76(\mathrm{~m}, 1 \mathrm{H}), 2.16(\mathrm{dd}, J=7.3,1.0 \mathrm{~Hz}, 2 \mathrm{H}), 5.62(\mathrm{~s}$, $1 \mathrm{H}), 5.67$ (d, $J=2.3 \mathrm{~Hz}, 1 \mathrm{H}), 6.50$ (brs, $1 \mathrm{H}), 7.05$ (d, $J=8.5 \mathrm{~Hz}, 1 \mathrm{H}), 7.14$ (ddd, $J=8.1,7.2,1.0 \mathrm{~Hz}$, 1H), 7.26 (brs, 1H), 7.52 (ddd, $J=7.9,7.2,1.6 \mathrm{~Hz}, 1 \mathrm{H}), 8.20$ (dd, $J=8.0,1.7 \mathrm{~Hz}, 1 \mathrm{H})$

${ }^{1} \mathrm{H}$ NMR (DMSO- $\left.d_{6}\right) \delta 0.83(\mathrm{~d}, J=6.6 \mathrm{~Hz}, 6 \mathrm{H}), 1.60$ (hept, $\left.J=6.7 \mathrm{~Hz}, 1 \mathrm{H}\right), 2.22(\mathrm{~d}, J=7.1 \mathrm{~Hz}, 2 \mathrm{H})$, $5.51(\mathrm{~d}, J=3.2 \mathrm{~Hz}, 1 \mathrm{H}), 5.79(\mathrm{~d}, J=3.1 \mathrm{~Hz}, 1 \mathrm{H}), 7.01-7.07(\mathrm{~m}, 1 \mathrm{H}), 7.35(\mathrm{dd}, J=8.3,0.6 \mathrm{~Hz}, 1 \mathrm{H})$, 7.50 (ddd, $J=8.1,7.1,1.6 \mathrm{~Hz}, 1 \mathrm{H}), 7.94$ (dd, $J=7.9,1.6 \mathrm{~Hz}, 1 \mathrm{H}), 8.82$ (s, 1H), 9.18 (s, 1H).

${ }^{13} \mathrm{C} \mathrm{NMR}\left(\mathrm{CDCl}_{3}\right) \delta 22.53,28.13,45.11,117.49,118.86,121.77,127.04,129.11,133.81,144.10,166.62$ ${ }^{11} \mathrm{~B}$ NMR $\left(\mathrm{CDCl}_{3}\right) \delta 28.91$

HRMS Calcd for $\mathrm{C}_{13} \mathrm{H}_{17} \mathrm{BN}_{2} \mathrm{ONa}:[\mathrm{M}+\mathrm{Na}]^{+}, 251.1326$. Found: $m / z 251.1325$ 
Structure Determination of 3dc (DMSO- $\left.\boldsymbol{d}_{6}\right)$.

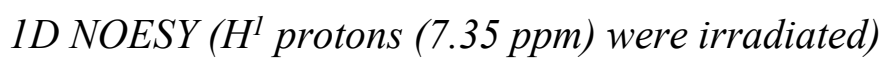

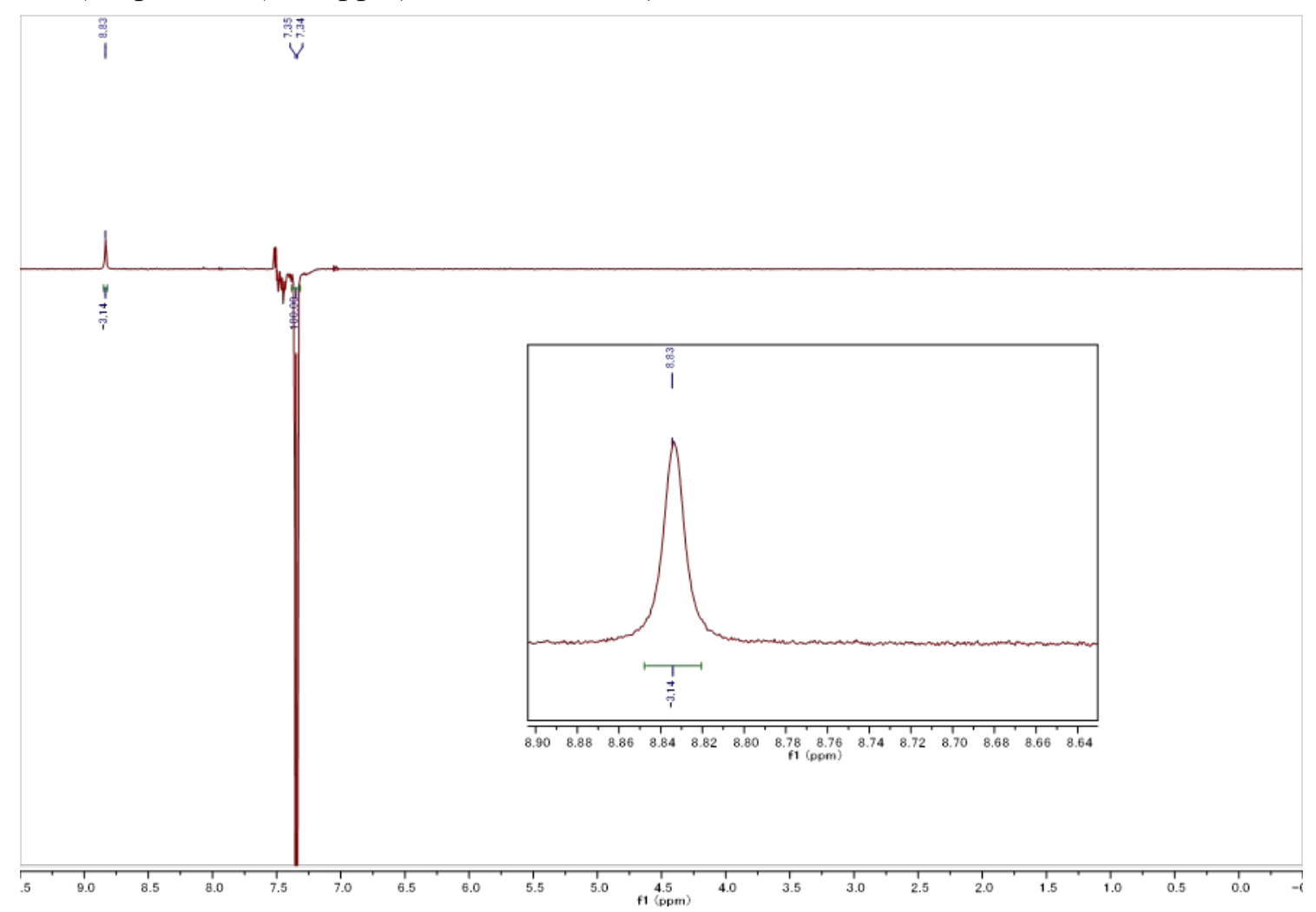

$8.83 \mathrm{ppm}(\mathrm{s}, \mathrm{H})$ was assigned to $\mathrm{H}^{2}$

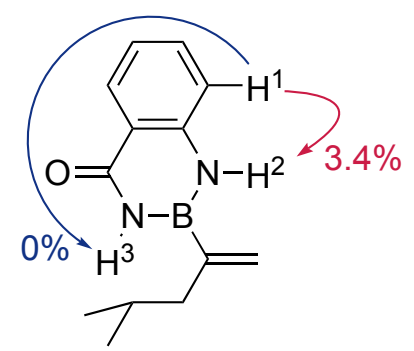


$1 D$ NOESY (H $H^{6}$ protons (2.21 ppm) were irradiated)
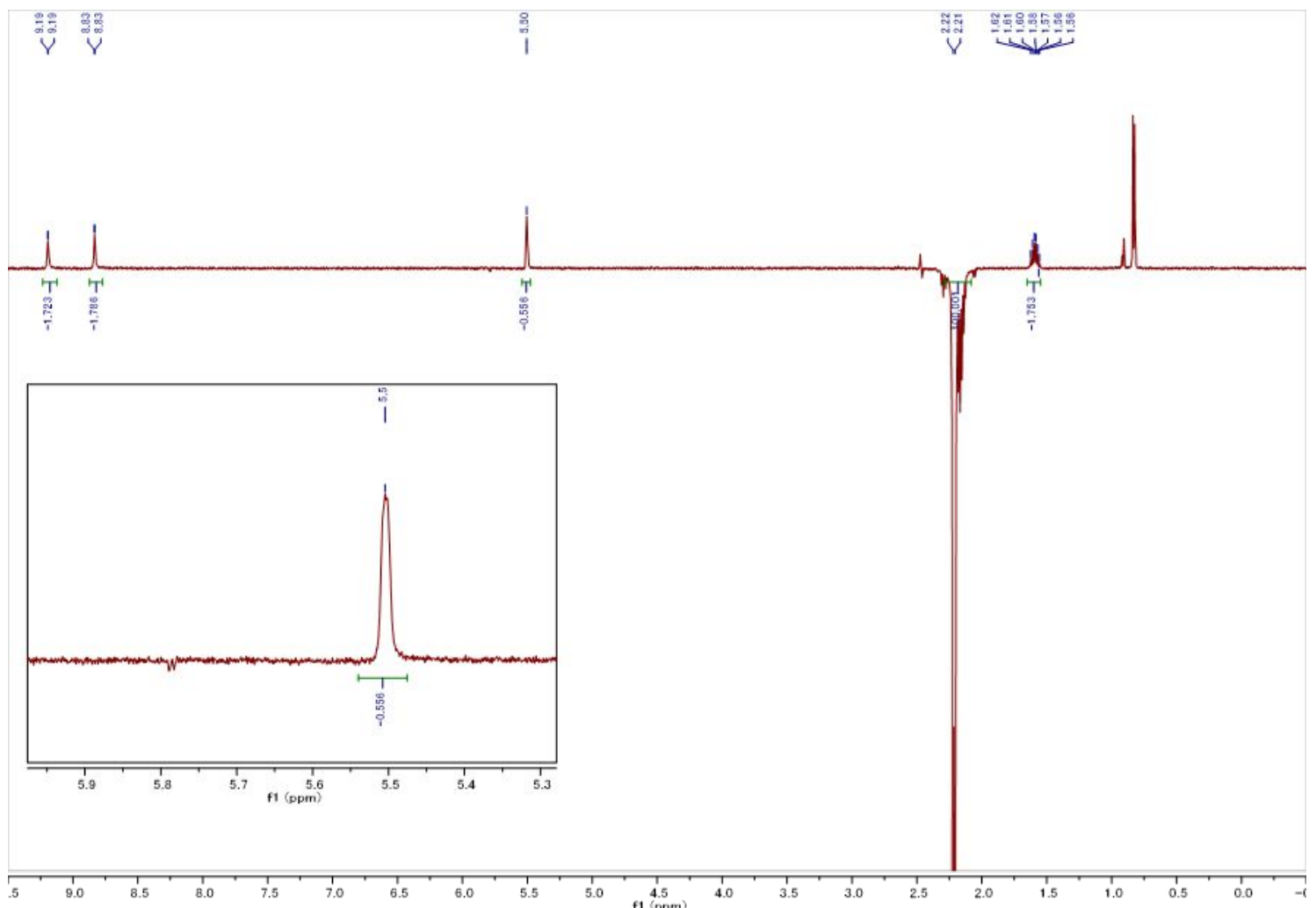

$5.50 \mathrm{ppm}(\mathrm{s}, \mathrm{H})$ was assigned to $\mathrm{H}^{5}$

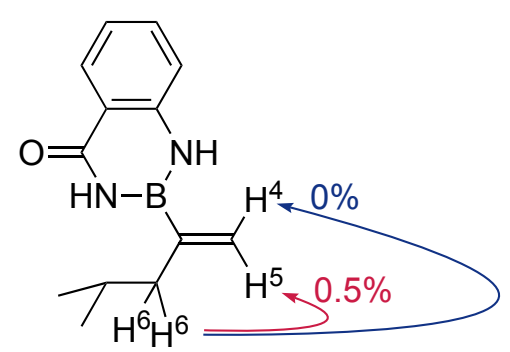


<smiles>C=C(Nc1ccccc1C(N)=O)C1CCCC1</smiles>

A pale yellow solid: $\mathrm{mp} 149-150{ }^{\circ} \mathrm{C}$

${ }^{1} \mathrm{H}$ NMR $\left(\mathrm{CDCl}_{3}\right) \delta 1.36-1.47(\mathrm{~m}, 2 \mathrm{H}), 1.58-1.69(\mathrm{~m}, 2 \mathrm{H}), 1.68-1.77(\mathrm{~m}, 2 \mathrm{H}), 1.85-1.92(\mathrm{~m}, 2 \mathrm{H})$, $2.60-2.71(\mathrm{~m}, 1 \mathrm{H}), 5.57(\mathrm{~s}, 1 \mathrm{H}), 5.63(\mathrm{~s}, 1 \mathrm{H}), 6.58(\mathrm{brs}, 1 \mathrm{H}), 7.05(\mathrm{~d}, J=8.1 \mathrm{~Hz}, 1 \mathrm{H}), 7.13(\mathrm{t}, J=7.6$ $\mathrm{Hz}, 1 \mathrm{H}), 7.31$ (brs, $1 \mathrm{H}), 7.51(\mathrm{td}, J=8.0,1.6 \mathrm{~Hz}, 1 \mathrm{H}), 8.20(\mathrm{dd}, J=7.9,1.5 \mathrm{~Hz}, 1 \mathrm{H})$

${ }^{13} \mathrm{C} \mathrm{NMR}\left(\mathrm{CDCl}_{3}\right) \delta 24.81,32.14,44.32,117.49,118.88,121.67,122.50,129.06,133.73,144.16,166.52$ ${ }^{11} \mathrm{~B}$ NMR $\left(\mathrm{CDCl}_{3}\right) \delta 29.23$

HRMS Calcd for $\mathrm{C}_{14} \mathrm{H}_{17} \mathrm{BN}_{2} \mathrm{ONa}$ : $[\mathrm{M}+\mathrm{Na}]^{+}, 263.1326$. Found: $m / z 263.1328$

\section{2-(3,3-dimethylbut-1-en-2-yl)-2,3-dihydrobenzo[d][1,3,2] diazaborinin-4(1H)-one (3fc)}<smiles>C=C(B1NC(=O)c2ccccc2N1)C(C)(C)C</smiles>

A pale yellow solid: $\mathrm{mp} 109-110{ }^{\circ} \mathrm{C}$

${ }^{1} \mathrm{H}$ NMR $\left(\mathrm{CDCl}_{3}\right) \delta 1.15(\mathrm{~s}, 9 \mathrm{H}), 5.28(\mathrm{~d}, J=1.7 \mathrm{~Hz}, 1 \mathrm{H}), 5.51-5.57$ (m, 1H), 6.42 (brs, 1H), 6.99 7.05 (m, 1H), 7.11 (brs, 1H), $7.12-7.17$ (m, 1H), 7.52 (ddd, $J=8.2,7.3,1.6 \mathrm{~Hz}, 1 \mathrm{H}), 8.21$ (dd, $J=8.0$, $1.5 \mathrm{~Hz}, 1 \mathrm{H})$.

${ }^{13} \mathrm{C} \mathrm{NMR}\left(\mathrm{CDCl}_{3}\right) \delta 30.31,35.92,117.45,118.76,119.91,121.76,129.07,133.75,144.08,166.24$ ${ }^{11} \mathrm{~B}$ NMR $\left(\mathrm{CDCl}_{3}\right) \delta 30.59$

HRMS Calcd for : $[2 \mathrm{M}+\mathrm{Na}]^{+}, 479.2760$. Found: $m / z 479.2766$

2-(1-(trimethylsilyl)vinyl)-2,3-dihydrobenzo $[d][1,3,2]$ diazaborinin-4(1H)-one (3gc)<smiles>C=C(S)B1NC(=O)c2ccccc2N1</smiles>

A pale yellow solid: mp $114-117^{\circ} \mathrm{C}$ 
${ }^{1} \mathrm{H}$ NMR $\left(\mathrm{CDCl}_{3}\right) \delta 0.18(\mathrm{~s}, 9 \mathrm{H}), 6.20(\mathrm{~d}, J=4.6 \mathrm{~Hz}, 1 \mathrm{H}), 6.35(\mathrm{brs}, 1 \mathrm{H}), 6.37(\mathrm{~d}, J=4.5 \mathrm{~Hz}, 1 \mathrm{H}), 7.01$ $(\mathrm{d}, J=8.0 \mathrm{~Hz}, 1 \mathrm{H}), 7.06$ (brs, $1 \mathrm{H}), 7.14$ (ddd, $J=8.1,7.2,1.1 \mathrm{~Hz}, 1 \mathrm{H}), 7.51$ (ddd, $J=8.1,7.2,1.6 \mathrm{~Hz}$, $1 \mathrm{H}), 8.21(\mathrm{dd}, J=7.9,1.7 \mathrm{~Hz}, 1 \mathrm{H})$

${ }^{13} \mathrm{C} \mathrm{NMR}\left(\mathrm{CDCl}_{3}\right) \delta-0.86,117.44,118.76,121.68,129.11,133.75,139.35,144.21,166.43$

${ }^{11} \mathrm{~B}$ NMR $\left(\mathrm{CDCl}_{3}\right) \delta 30.59$

HRMS Calcd for $\mathrm{C}_{12} \mathrm{H}_{18} \mathrm{BN}_{2} \mathrm{O}_{2} \mathrm{Si}:[\mathrm{M}+\mathrm{H}]^{+}, 245.1276$. Found: $m / z 245.1276$

\section{2-(5-chloropent-1-en-2-yl)-2,3-dihydrobenzo[d] [1,3,2] diazaborinin-4(1H)-one (3hc)}

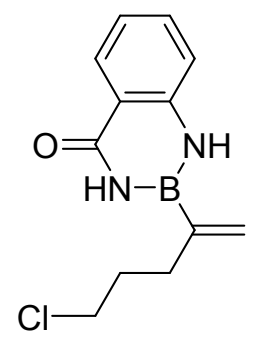

A pale yellow solid: mp $139-140{ }^{\circ} \mathrm{C}$

${ }^{1} \mathrm{H} \mathrm{NMR}\left(\mathrm{CDCl}_{3}\right) \delta 1.90-2.02(\mathrm{~m}, 2 \mathrm{H}), 2.46(\mathrm{t}, J=7.4 \mathrm{~Hz}, 2 \mathrm{H}), 3.61(\mathrm{t}, J=6.2 \mathrm{~Hz}, 2 \mathrm{H}), 5.72(\mathrm{~s}, 2 \mathrm{H})$, 6.59 (brs, 1H), 7.05 (ddd, $J=8.1,1.1,0.5 \mathrm{~Hz}, 1 \mathrm{H}), 7.16$ (ddd, $J=8.1,7.2,1.1 \mathrm{~Hz}, 1 \mathrm{H}), 7.26$ (brs, 1H), $7.53(\mathrm{ddd}, J=8.1,7.2,1.6 \mathrm{~Hz}, 1 \mathrm{H}), 8.21(\mathrm{dd}, J=7.9,1.6 \mathrm{~Hz}, 1 \mathrm{H})$

${ }^{13} \mathrm{C} \mathrm{NMR}\left(\mathrm{CDCl}_{3}\right) \delta 31.77,32.01,44.55,117.56,118.96,121.93,126.98,129.11,133.86,144.00,166.51$

${ }^{11} \mathrm{~B}$ NMR $\left(\mathrm{CDCl}_{3}\right) \delta 28.64$

HRMS Calcd for $\mathrm{C}_{12} \mathrm{H}_{13} \mathrm{BN}_{2} \mathrm{ONaCl}$ : $[\mathrm{M}+\mathrm{Na}]^{+}$, 271.0780. Found: $m / z 271.0780$

\section{5-(4-oxo-3,4-dihydrobenzo[d] $[1,3,2]$ diazaborinin-2(1H)-yl)hex-5-enenitrile (3ic)}

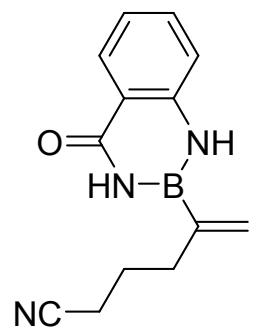

A pale yellow solid: $\mathrm{mp} 127-128^{\circ} \mathrm{C}$

${ }^{1} \mathrm{H} \mathrm{NMR}\left(\mathrm{CDCl}_{3}\right) \delta 1.80-1.91(\mathrm{~m}, 2 \mathrm{H}), 2.40(\mathrm{t}, J=7.0 \mathrm{~Hz}, 2 \mathrm{H}), 2.49(\mathrm{t}, J=7.5 \mathrm{~Hz}, 2 \mathrm{H}), 5.73(\mathrm{~d}, J=$ $1.7 \mathrm{~Hz}, 1 \mathrm{H}), 5.80$ (d, $J=1.1 \mathrm{~Hz}, 1 \mathrm{H}), 6.76$ (brs, 1H), 7.09 (dd, $J=8.2,1.0 \mathrm{~Hz}, 1 \mathrm{H}), 7.14$ (ddd, $J=8.1$, $7.2,1.1 \mathrm{~Hz}, 1 \mathrm{H}), 7.52$ (ddd, $J=8.1,7.2,1.6 \mathrm{~Hz}, 1 \mathrm{H}), 7.92$ (brs, $1 \mathrm{H}), 8.19$ (dd, $J=7.9,1.6 \mathrm{~Hz}, 1 \mathrm{H})$ ${ }^{13} \mathrm{C} \mathrm{NMR}\left(\mathrm{CDCl}_{3}\right) \delta 16.66,24.75,33.62,117.64,118.95,119.59,121.98,127.51,129.00,133.90,143.99$, 166.70

${ }^{11} \mathrm{~B} \mathrm{NMR}\left(\mathrm{CDCl}_{3}\right) \delta 28.66$

HRMS Calcd for $\mathrm{C}_{17} \mathrm{H}_{14} \mathrm{BN}_{3} \mathrm{ONa}$ : $[\mathrm{M}+\mathrm{Na}]^{+}, 262.1122$. Found: $m / z 262.1124$ 


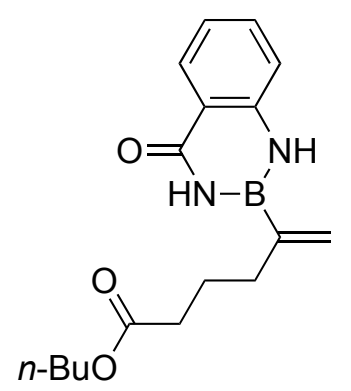

A pale yellow solid: $\mathrm{mp} 119-120^{\circ} \mathrm{C}$

${ }^{1} \mathrm{H}$ NMR $\left(\mathrm{CDCl}_{3}\right) \delta 0.95(\mathrm{t}, J=7.4 \mathrm{~Hz}, 3 \mathrm{H}), 1.36-1.46(\mathrm{~m}, 2 \mathrm{H}), 1.59-1.68(\mathrm{~m}, 2 \mathrm{H}), 1.72-1.81(\mathrm{~m}$, $2 \mathrm{H}), 2.30(\mathrm{t}, J=8.1 \mathrm{~Hz}, 2 \mathrm{H}), 2.42(\mathrm{t}, J=6.4 \mathrm{~Hz}, 2 \mathrm{H}), 4.17(\mathrm{t}, J=6.7 \mathrm{~Hz}, 2 \mathrm{H}), 5.66(\mathrm{~s}, 1 \mathrm{H}), 5.68(\mathrm{~s}, 1 \mathrm{H})$, 7.13 (ddd, $J=8.1,7.1,1.1 \mathrm{~Hz}, 1 \mathrm{H}), 7.19$ (dd, $J=8.2,1.0 \mathrm{~Hz}, 1 \mathrm{H}), 7.37$ (brs, 1H), 7.52 (ddd, $J=8.1,7.1$, $1.5 \mathrm{~Hz}, 1 \mathrm{H}), 7.72$ (brs, $1 \mathrm{H}), 8.20$ (dd, $J=8.0,1.6 \mathrm{~Hz}, 1 \mathrm{H})$

${ }^{13} \mathrm{C} \mathrm{NMR}\left(\mathrm{CDCl}_{3}\right) \delta 13.72,19.15,24.75,30.67,33.06,35.03,64.63,117.88,118.95,121.53,126.45$, 128.91, 133.65, 144.67, 166.67, 174.62

${ }^{11} \mathrm{~B} \mathrm{NMR}\left(\mathrm{CDCl}_{3}\right) \delta 28.49$

HRMS Calcd for $\mathrm{C}_{17} \mathrm{H}_{23} \mathrm{BN}_{2} \mathrm{O}_{3} \mathrm{Na}$ : [M+Na] $]^{+}$, 337.1694. Found: $m / z 337.1697$

2-(6-((tert-butyldimethylsilyl)oxy)hex-1-en-2-yl)-2,3-dihydrobenzo[d][1,3,2]diazaborinin-4(1H)one (3kc)

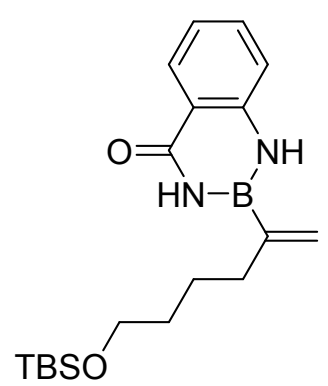

A pale yellow solid: $\mathrm{mp} 120-121^{\circ} \mathrm{C}$

${ }^{1} \mathrm{H}$ NMR $\left(\mathrm{CDCl}_{3}\right) \delta 0.04(\mathrm{~s}, 6 \mathrm{H}), 0.88(\mathrm{~s}, 9 \mathrm{H}), 1.49-1.57(\mathrm{~m}, 4 \mathrm{H}), 2.30(\mathrm{t}, J=6.9 \mathrm{~Hz}, 2 \mathrm{H}), 3.64(\mathrm{t}, J=$ $6.0 \mathrm{~Hz}, 2 \mathrm{H}), 5.64(\mathrm{~s}, 1 \mathrm{H}), 5.66(\mathrm{~s}, 1 \mathrm{H}), 6.52$ (brs, $1 \mathrm{H}), 7.04(\mathrm{~d}, J=7.8 \mathrm{~Hz}, 1 \mathrm{H}), 7.14$ (ddd, J = 8.1, 7.2, $1.1 \mathrm{~Hz}, 1 \mathrm{H}), 7.23$ (brs, $1 \mathrm{H}), 7.52(\mathrm{ddd}, J=8.1,7.2,1.6 \mathrm{~Hz}, 1 \mathrm{H}), 8.21(\mathrm{dd}, J=8.0,1.7 \mathrm{~Hz}, 1 \mathrm{H})$

${ }^{13} \mathrm{C}$ NMR $\left(\mathrm{CDCl}_{3}\right) \delta-5.25,18.36,25.66,25.95,32.37,34.98,62.96,117.49,118.94,121.76,125.80$, $129.11,133.76,144.08,166.45$

${ }^{11} \mathrm{~B}$ NMR $\left(\mathrm{CDCl}_{3}\right) \delta 28.83$

HRMS Calcd for $\mathrm{C}_{19} \mathrm{H}_{32} \mathrm{BN}_{2} \mathrm{O}_{2} \mathrm{Si}:[\mathrm{M}+\mathrm{H}]^{+}, 359.2321$. Found: $m / z 359.2330$ 


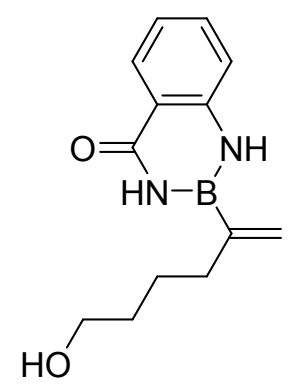

A pale yellow solid: $\mathrm{mp} 129-130{ }^{\circ} \mathrm{C}$

${ }^{1} \mathrm{H}$ NMR $\left(\mathrm{CDCl}_{3}\right) \delta 1.42(\mathrm{t}, J=5.2 \mathrm{~Hz}, 1 \mathrm{H}), 1.55-1.68(\mathrm{~m}, 4 \mathrm{H}), 2.28-2.38(\mathrm{~m}, 2 \mathrm{H}), 3.68-3.77(\mathrm{~m}$, 2H), $5.65(\mathrm{~s}, 1 \mathrm{H}), 5.67$ (s, 1H), $6.72(\mathrm{brs}, 1 \mathrm{H}), 7.04$ (dd, $J=8.2,1.1 \mathrm{~Hz}, 1 \mathrm{H}), 7.14$ (ddd, $J=8.1,7.2,1.1$ $\mathrm{Hz}, 1 \mathrm{H}), 7.31$ (brs, $1 \mathrm{H}), 7.51$ (ddd, $J=8.0,7.2,1.6 \mathrm{~Hz}, 1 \mathrm{H}), 8.20(\mathrm{dd}, J=7.9,1.7 \mathrm{~Hz}, 1 \mathrm{H})$ ${ }^{13} \mathrm{C} \mathrm{NMR}\left(\mathrm{CDCl}_{3}\right) \delta 25.61,31.81,34.76,62.51,116.37,117.54,118.94,121.74,125.93,129.09,133.76$, $144.18,166.53$

${ }^{11} \mathrm{~B}$ NMR $\left(\mathrm{CDCl}_{3}\right) \delta 28.69$

HRMS Calcd for $\mathrm{C}_{13} \mathrm{H}_{18} \mathrm{BN}_{2} \mathrm{O}_{2}$ : $[\mathrm{M}+\mathrm{H}]^{+}, 245.1456$. Found: $m / z 245.1453$

\section{2-(1-phenylvinyl)-2,3-dihydrobenzo[ $[d][1,3,2]$ diazaborinin-4(1H)-one (3mc)}<smiles>C=C(B1NC(=O)c2ccccc2N1)c1ccccc1</smiles>

A pale yellow solid: $\mathrm{mp} 153-154{ }^{\circ} \mathrm{C}$

${ }^{1} \mathrm{H}$ NMR $\left(\mathrm{CDCl}_{3}\right) \delta 5.89(\mathrm{~d}, J=1.9 \mathrm{~Hz}, 1 \mathrm{H}), 6.00(\mathrm{~d}, J=1.9 \mathrm{~Hz}, 1 \mathrm{H}), 6.51$ (brs, $\left.1 \mathrm{H}\right), 7.00(\mathrm{~d}, J=8.1 \mathrm{~Hz}$, $1 \mathrm{H}), 7.16(\mathrm{ddd}, J=8.1,7.2,1.1 \mathrm{~Hz}, 1 \mathrm{H}), 7.27-7.38(\mathrm{~m}, 4 \mathrm{H}), 7.38-7.44$ (m, 2H), 7.52 (ddd, $J=8.4$, $7.2,1.6 \mathrm{~Hz}, 1 \mathrm{H}), 8.23(\mathrm{~d}, J=7.9 \mathrm{~Hz}, 1 \mathrm{H})$

${ }^{13} \mathrm{C} \mathrm{NMR}\left(\mathrm{CDCl}_{3}\right) \delta 117.58,119.02,121.98,127.43,127.49,127.66,128.88,129.15,133.83,141.51$, $143.97,166.38$

${ }^{11} \mathrm{~B}$ NMR $\left(\mathrm{CDCl}_{3}\right) \delta 29.04$

HRMS Calcd for $\mathrm{C}_{15} \mathrm{H}_{13} \mathrm{BN}_{2} \mathrm{ONa}$ : $[\mathrm{M}+\mathrm{Na}]^{+}, 271.1013$. Found: $m / z 271.1014$

2-(1-(4-bromophenyl)vinyl)-2,3-dihydrobenzo[d][1,3,2] diazaborinin-4(1H)-one (3nc) 


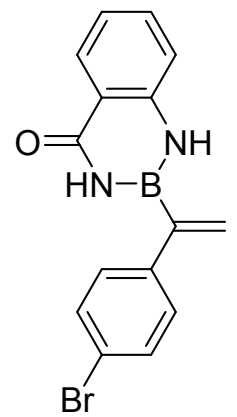

A pale yellow solid: $\mathrm{mp} 218-220^{\circ} \mathrm{C}$

${ }^{1} \mathrm{H}$ NMR $\left(\mathrm{CDCl}_{3}\right) \delta 5.89(\mathrm{~d}, J=1.7 \mathrm{~Hz}, 1 \mathrm{H}), 5.99$ (d, $\left.J=1.7 \mathrm{~Hz}, 1 \mathrm{H}\right), 6.44$ (brs, $\left.1 \mathrm{H}\right), 7.00$ (d, $J=8.5 \mathrm{~Hz}$, 1H), $7.12-7.22$ (m, 3H), 7.24 (brs, $1 \mathrm{H}), 7.47-7.56$ (m, 3H), 8.22 (dd, $J=7.9,1.5 \mathrm{~Hz}, 1 \mathrm{H})$.

${ }^{13} \mathrm{C} \mathrm{NMR}\left(\mathrm{CDCl}_{3}\right) \delta 117.60,119.06,121.80,122.15,127.87,129.16,129.19,132.01,133.91,140.41$, $143.85,166.28$

${ }^{11} \mathrm{~B}$ NMR $\left(\mathrm{CDCl}_{3}\right) \delta 28.93$

HRMS Calcd for $\mathrm{C}_{15} \mathrm{H}_{13} \mathrm{BN}_{2} \mathrm{OBr}$ : [M+H]+, 327.0299. Found: $m / z 327.0302$

\section{2-(1-(2-methoxyphenyl)vinyl)-2,3-dihydrobenzo $[d][1,3,2]$ diazaborinin-4(1H)-one (3oc)}

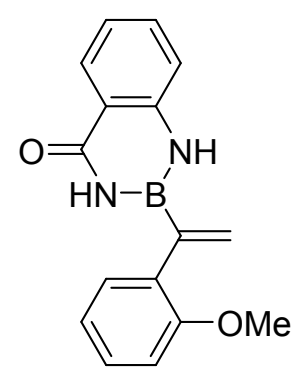

A pale yellow solid: $\mathrm{mp} 188-189^{\circ} \mathrm{C}$

${ }^{1} \mathrm{H}$ NMR $\left(\mathrm{CDCl}_{3}\right) \delta 3.69$ (s, 3H), $5.81(\mathrm{~d}, J=2.3 \mathrm{~Hz}, 1 \mathrm{H}), 5.88(\mathrm{~s}, 1 \mathrm{H}), 6.47$ (brs, $\left.1 \mathrm{H}\right), 6.89(\mathrm{~d}, J=8.2$ $\mathrm{Hz}, 1 \mathrm{H}), 6.96-7.05(\mathrm{~m}, 2 \mathrm{H}), 7.09-7.16(\mathrm{~m}, 2 \mathrm{H}), 7.27(\mathrm{~d}, J=8.7 \mathrm{~Hz}, 1 \mathrm{H}), 7.33(\mathrm{td}, J=7.8,1.7 \mathrm{~Hz}, 1 \mathrm{H})$, $7.50(\mathrm{td}, J=7.8,7.4,1.0 \mathrm{~Hz}, 1 \mathrm{H}), 8.21(\mathrm{dd}, J=8.0,1.5 \mathrm{~Hz}, 1 \mathrm{H})$

${ }^{13} \mathrm{C}$ NMR $\left(\mathrm{CDCl}_{3}\right) \delta 55.55,110.85,117.41,118.82,121.39,121.45,126.73,129.11,129.16,129.27$, $131.14,133.65,144.34,156.10,166.57$

${ }^{11} \mathrm{~B}$ NMR $\left(\mathrm{CDCl}_{3}\right) \delta 29.27$

HRMS Calcd for $\mathrm{C}_{16} \mathrm{H}_{16} \mathrm{BN}_{2} \mathrm{O}_{2}:[\mathrm{M}+\mathrm{H}]^{+}$, 279.1299. Found: $m / z 279.1301$ 


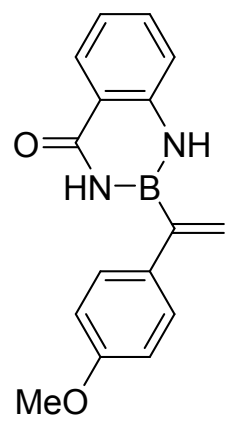

A pale yellow solid: $\mathrm{mp} 166-167^{\circ} \mathrm{C}$

${ }^{1} \mathrm{H} \mathrm{NMR}\left(\mathrm{CDCl}_{3}\right) \delta 3.84(\mathrm{~s}, 3 \mathrm{H}), 5.79(\mathrm{~d}, J=1.8 \mathrm{~Hz}, 1 \mathrm{H}), 5.94(\mathrm{~d}, J=1.9 \mathrm{~Hz}, 1 \mathrm{H}), 6.55$ (brs, $\left.1 \mathrm{H}\right), 6.92$ $(\mathrm{d}, J=8.7 \mathrm{~Hz}, 2 \mathrm{H}), 7.00(\mathrm{~d}, J=8.1 \mathrm{~Hz}, 1 \mathrm{H}), 7.15(\mathrm{t}, J=7.2 \mathrm{~Hz}, 1 \mathrm{H}), 7.27(\mathrm{~d}, J=8.8 \mathrm{~Hz}, 2 \mathrm{H}), 7.32$ (brs, $1 \mathrm{H}), 7.51(\mathrm{ddd}, J=8.4,7.2,1.6 \mathrm{~Hz}, 1 \mathrm{H}), 8.23(\mathrm{dd}, J=8.0,1.6 \mathrm{~Hz}, 1 \mathrm{H})$

${ }^{13} \mathrm{C}$ NMR $\left(\mathrm{CDCl}_{3}\right) \delta 55.35,114.23,117.56,119.02,121.91,125.88,128.63,129.13,133.79,133.83$, $144.01,159.27,166.41$

${ }^{11} \mathrm{~B}$ NMR $\left(\mathrm{CDCl}_{3}\right) \delta 29.32$

HRMS Calcd for $\mathrm{C}_{16} \mathrm{H}_{16} \mathrm{BN}_{2} \mathrm{O}_{2}$ : $[\mathrm{M}+\mathrm{H}]^{+}, 279.1299$. Found: $m / z 279.1304$

\section{2-(1-(3,4,5-trimethoxyphenyl)vinyl)-2,3-dihydrobenzo[ $[d][1,3,2]$ diazaborinin-4(1H)-one (3qc)}

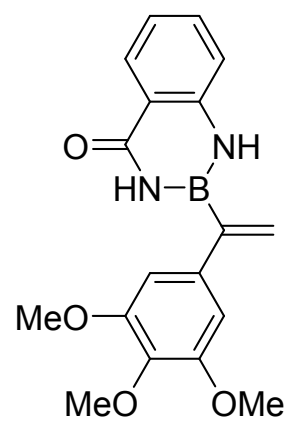

A pale yellow solid: $\mathrm{mp} 164-165^{\circ} \mathrm{C}$

${ }^{1} \mathrm{H} \mathrm{NMR}\left(\mathrm{CDCl}_{3}\right) \delta 3.87$ (s, 6H), 3.89 (s, 3H), 5.86 (s, 1H), 5.97 (s, 1H), 6.50 (s, 2H), 6.54 (brs, 1H), 7.02 $(\mathrm{d}, J=8.1 \mathrm{~Hz}, 1 \mathrm{H}), 7.17(\mathrm{t}, J=8.3,7.0 \mathrm{~Hz}, 1 \mathrm{H}), 7.30$ (brs, $1 \mathrm{H}), 7.53$ (td, $J=7.5,6.7,1.1 \mathrm{~Hz}, 1 \mathrm{H}), 8.23$ $(\mathrm{dd}, J=7.9,1.5 \mathrm{~Hz}, 1 \mathrm{H})$

${ }^{13} \mathrm{C}$ NMR $\left(\mathrm{CDCl}_{3}\right) \delta 56.22,60.94,104.61,117.59,119.06,122.07,127.03,129.18,133.91,137.32$, $137.69,143.93,153.45,166.32$

${ }^{11} \mathrm{~B} \mathrm{NMR}\left(\mathrm{CDCl}_{3}\right) \delta 29.12$

HRMS Calcd for $\mathrm{C}_{18} \mathrm{H}_{19} \mathrm{BN}_{2} \mathrm{O}_{4}:[\mathrm{M}+\mathrm{H}]^{+}, 339.1511$. Found: $m / z 339.1512$

\section{1,3-dimethyl-2-(oct-1-en-2-yl)-2,3-dihydro-1H-naphtho[1,8-de][1,3,2]diazaborinine (3ad)}

A Schlenk tube equipped with a magnetic stirring bar was charged with $\mathrm{SIPrCuCl}(7.5 \mu \mathrm{mol}), t$-BuOK (1.0 M solution in THF, $9.0 \mu \mathrm{mol})$ and 1,4-dioxane $(0.5 \mathrm{~mL})$ before the mixture was stirred at room temperature for $10 \mathrm{~min}$. To the mixture was added (pin)B-B(mdan) $(0.18 \mathrm{mmol}), \mathrm{MeOH}(0.45 \mathrm{mmol})$ and 1-octyne $(0.15 \mathrm{mmol})$, and the resulting mixture was stirred at $50{ }^{\circ} \mathrm{C}$ for $3 \mathrm{~h}$. The mixture was diluted 
with ethyl acetate and filtered through a Celite plug. The organic solution was washed with brine, dried over $\mathrm{Na}_{2} \mathrm{SO}_{4}$, and evaporated. Purification of the residue by silica gel-column chromatography ${ }^{20}$ (hexane/ethyl acetate as an eluent) gave the product.

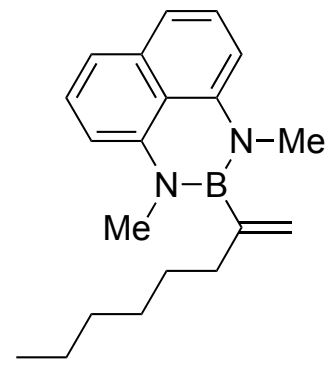

A pale yellow oil (NMR yield 64\%, Isolated yield 57\%)

${ }^{1} \mathrm{H} \mathrm{NMR}\left(\mathrm{CDCl}_{3}\right) \delta 0.89(\mathrm{t}, J=6.9 \mathrm{~Hz}, 3 \mathrm{H}), 1.30-1.43(\mathrm{~m}, 6 \mathrm{H}), 1.43-1.55(\mathrm{~m}, 2 \mathrm{H}), 2.29(\mathrm{t}, J=8.2 \mathrm{~Hz}$, 2H), $3.07(\mathrm{~s}, 6 \mathrm{H}), 5.18(\mathrm{~d}, J=2.9 \mathrm{~Hz}, 1 \mathrm{H}), 5.54-5.60(\mathrm{~m}, 1 \mathrm{H}), 6.51(\mathrm{td}, J=7.6,1.0 \mathrm{~Hz}, 2 \mathrm{H}), 7.21(\mathrm{~d}, J$ $=8.3 \mathrm{~Hz}, 2 \mathrm{H}), 7.31(\mathrm{t}, J=7.9 \mathrm{~Hz}, 2 \mathrm{H})$.

${ }^{13} \mathrm{C} \mathrm{NMR}\left(\mathrm{CDCl}_{3}\right) \delta 14.06,22.64,28.64,29.58,31.80,35.08,37.73,103.35,117.91,120.13,120.30$, $127.20,135.81,142.98$.

${ }^{11} \mathrm{~B} \mathrm{NMR}\left(\mathrm{CDCl}_{3}\right) \delta 31.08$

HRMS Calcd for $\mathrm{C}_{20} \mathrm{H}_{28} \mathrm{BN}_{2}$ : $[\mathrm{M}+\mathrm{H}]^{+}$, 307.2340. Found: $m / z$ 307.2346

\section{One Pot Sequential Internal-Selective Hydroboration-Suzuki-Miyaura Cross-Coupling}

\section{Synthesis of (1-([1,1'-biphenyl]-4-yl)vinyl)trimethylsilane (4a).}<smiles>C=C(C)c1ccc(-c2ccccc2)cc1</smiles>

A Schrenk tube equipped with a magnetic stirring bar was charged with $6 \mathrm{DippCuCl}(7.5 \mu \mathrm{mol}), t-\mathrm{BuOK}$ $(1.0 \mathrm{M}$ solution in THF, $9.0 \mu \mathrm{mol})$ and 1,4-dioxane $(0.5 \mathrm{~mL})$ before the mixture was stirred at room temperature for $10 \mathrm{~min}$. To the mixture was added (pin)B-B(aam) $(0.18 \mathrm{mmol}), \mathrm{MeOH}(0.45 \mathrm{mmol})$ and trimethylsilylacetylene $(0.15 \mathrm{mmol})$, and the resulting mixture was stirred at room temperature for $24 \mathrm{~h}$. To the mixture were added 4-bromobiphenyl (0.15 mmol), $\mathrm{K}_{3} \mathrm{PO}_{4}(1.125 \mathrm{mmol}), \mathrm{Pd}(\mathrm{OAc})_{2}(7.5 \mu \mathrm{mol})$, Sphos $(15.0 \mu \mathrm{mol}), 1,4$-dioxane $(1.4 \mathrm{~mL})$ and $\mathrm{H}_{2} \mathrm{O}(0.38 \mathrm{~mL})$, and the resulting mixture was stirred at 100 ${ }^{\circ} \mathrm{C}$ for $24 \mathrm{~h}$. The mixture was diluted with ethyl acetate and filtered through a Celite plug. The organic solution was washed with brine, dried over $\mathrm{MgSO}_{4}$, and evaporated. Purification of the residue by silica gel-column chromatography (hexane/ethyl acetate $=10: 1$ as an eluent) gave the product.

A white yellow solid: $\mathrm{mp} 80-82{ }^{\circ} \mathrm{C}$ 
${ }^{1} \mathrm{H} \mathrm{NMR}\left(\mathrm{CDCl}_{3}\right) \delta 0.24(\mathrm{~s}, 9 \mathrm{H}), 5.67(\mathrm{~d}, J=3.0 \mathrm{~Hz}, 1 \mathrm{H}), 5.92(\mathrm{~d}, J=2.9 \mathrm{~Hz}, 1 \mathrm{H}), 7.27-7.32(\mathrm{~m}, 2 \mathrm{H})$, $7.32-7.40(\mathrm{~m}, 1 \mathrm{H}), 7.42-7.50(\mathrm{~m}, 2 \mathrm{H}), 7.54-7.60(\mathrm{~m}, 2 \mathrm{H}), 7.60-7.67(\mathrm{~m}, 2 \mathrm{H})$.

${ }^{13} \mathrm{C} \mathrm{NMR}\left(\mathrm{CDCl}_{3}\right) \delta-0.79,126.86,126.95,127.09,127.13,127.17,127.20,127.25,128.73,128.75$, $139.09,140.94,143.73,152.92$.

HRMS Calcd for $\mathrm{C}_{17} \mathrm{H}_{21} \mathrm{Si}:[\mathrm{M}+\mathrm{H}]^{+}, 253.1407$. Found: $m / z 253.1411$

\section{Synthesis of iso-Combretastatin A4 (4b). ${ }^{21}$}<smiles>C=C(c1ccc(OC)c(O)c1)c1cc(OC)c(OC)c(OC)c1</smiles>

A reaction tube equipped with a magnetic stirring bar was charged with $6 \mathrm{DippCuCl}(7.5 \mu \mathrm{mol}), t-\mathrm{BuOK}$ (1.0 M solution in THF, $9.0 \mu \mathrm{mol})$ and 1,4-dioxane $(0.5 \mathrm{~mL})$ before the mixture was stirred at room temperature for $10 \mathrm{~min}$. To the mixture was added (pin)B-B(aam) $(0.18 \mathrm{mmol}), \mathrm{MeOH}(0.45 \mathrm{mmol})$ and 5-ethynyl-1,2,3-trimethoxybenzene $(0.15 \mathrm{mmol})$, and the resulting mixture was stirred at $50{ }^{\circ} \mathrm{C}$ for $3 \mathrm{~h}$. To the mixture were added 5-bromo-2-methoxyphenol (0.15 mmol), $\mathrm{K}_{3} \mathrm{PO}_{4}(1.125 \mathrm{mmol}), \mathrm{Pd}(\mathrm{OAc})_{2}(7.5$ $\mu \mathrm{mol})$, Sphos $(15.0 \mu \mathrm{mol}), 1,4-$ dioxane $(1.4 \mathrm{~mL})$ and $\mathrm{H}_{2} \mathrm{O}(0.38 \mathrm{~mL})$, and the reaction tube was set into a microwave reactor and heated to $140{ }^{\circ} \mathrm{C}$ for $30 \mathrm{~min}$. The mixture was diluted with ethyl acetate and filtered through a Celite plug. The organic solution was washed with brine, dried over $\mathrm{MgSO}_{4}$, and evaporated. Purification of the residue by silica gel-column chromatography (hexane/ethyl acetate $=2: 1$ as an eluent) gave the product.

${ }^{1} \mathrm{H} \mathrm{NMR}\left(\mathrm{CDCl}_{3}\right) \delta 3.82(\mathrm{~s}, 6 \mathrm{H}), 3.87(\mathrm{~s}, 3 \mathrm{H}), 3.91(\mathrm{~s}, 3 \mathrm{H}), 5.31(\mathrm{~d}, J=1.3 \mathrm{~Hz}, 1 \mathrm{H}), 5.38(\mathrm{~d}, J=1.4 \mathrm{~Hz}$, 1H), 5.59 (s, 1H), 6.55 (s, 2H), $6.79-6.87(\mathrm{~m}, 3 \mathrm{H}), 6.97$ (d, $J=2.1 \mathrm{~Hz}, 1 \mathrm{H})$.

${ }^{13} \mathrm{C} \mathrm{NMR}\left(\mathrm{CDCl}_{3}\right) \delta 55.96,56.11,60.91,105.66,110.11,112.87,114.43,120.17,134.65,137.43,137.64$, $145.15,146.38,149.51,152.77$.

\section{Synthesis of 6Dipp•HCl. ${ }^{22}$}

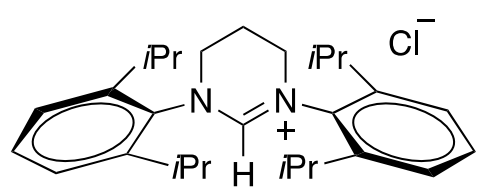

$N, N$-Diisopropylethylamine $(2.5 \mathrm{~mL}, 15 \mathrm{mmol})$ was added to a mixture of $N, N^{\prime}$-bis(2,6diisopropylphenyl)formamidine $(5.1 \mathrm{~g}, 14 \mathrm{mmol})$ and 1,3-dichloropropane $(6.6 \mathrm{~mL}, 69 \mathrm{mmol})$. The mixture was stirred at $120{ }^{\circ} \mathrm{C}$ for $16 \mathrm{~h}$ by using a reflux condenser. Excess 1,3-dichloropropane was then removed in vacuo. The resulting brown solid was dissolved in DCM $(20 \mathrm{~mL})$, washed with saturated aqueous $\mathrm{K}_{2} \mathrm{CO}_{3}(3 \times 10 \mathrm{~mL})$ and $\mathrm{H}_{2} \mathrm{O}(1 \times 10 \mathrm{~mL})$, dried over $\mathrm{MgSO}_{4}$, and concentrated in vacuo. This 
resulting brown solid was dissolved in DCM $(30 \mathrm{~mL})$ and toluene $(10 \mathrm{~mL})$ before the DCM was removed in vacuo. The resulting in a colorless precipitate was collected on a frit, washed with 2 portions of toluene $(5 \mathrm{~mL})$, and dried overnight under vacuum to give the target compound (4.06 g, 66\%) a colorless solid. ${ }^{1} \mathrm{H} \mathrm{NMR}\left(\mathrm{CDCl}_{3}\right) \delta 1.23(\mathrm{~d}, J=6.8 \mathrm{~Hz}, 12 \mathrm{H}), 1.38(\mathrm{~d}, J=6.8 \mathrm{~Hz}, 12 \mathrm{H}), 2.82(\mathrm{t}, J=5.7 \mathrm{~Hz}, 2 \mathrm{H}), 3.06$ (sept, $J=6.8 \mathrm{~Hz}, 4 \mathrm{H}), 4.27(\mathrm{t}, J=5.7 \mathrm{~Hz}, 4 \mathrm{H}), 7.26(\mathrm{~d}, J=7.8 \mathrm{~Hz}, 4 \mathrm{H}), 7.44$ (t, $J=7.8 \mathrm{~Hz}, 2 \mathrm{H}), 7.53$ (s, $1 \mathrm{H})$

${ }^{13} \mathrm{C} \mathrm{NMR}\left(\mathrm{CDCl}_{3}\right) \delta 19.39,24.79,24.81,28.85,49.11,125.12,131.19,135.86,145.67,152.84$

\section{Synthesis of 6DippCuCl. ${ }^{22}$}

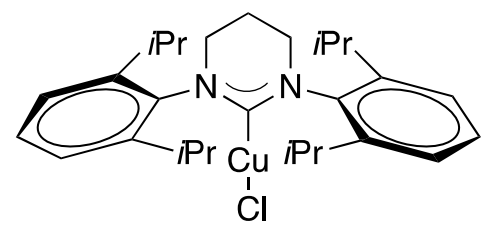

NaHMDS (1.9 M solution in THF, $1.2 \mathrm{~mL}, 2.25 \mu \mathrm{mol})$ was added to a solution of $6 \mathrm{Dipp} \cdot \mathrm{HCl}(0.970 \mathrm{~g}$, $2.20 \mathrm{mmol})$ in THF (10 mL) with stirring. After $2 \mathrm{~h}, \mathrm{CuCl}(0.223 \mathrm{~g}, 2.25 \mathrm{mmol})$ was added, and the mixture was allowed to stir overnight before being filtered through Celite, washed with DCM, and concentrated in vacuo to give a colorless solid. This was dissolved in a minimal amount of DCM, layered with hexane and placed in the freezer overnight. The resulting colorless solid was collected on a frit, and dried in vacuo to afford $(0.806 \mathrm{~g}, 73 \%)$ the title complex.

${ }^{1} \mathrm{H} \mathrm{NMR}\left(\mathrm{CDCl}_{3}\right) \delta 1.31(\mathrm{~d}, J=6.9 \mathrm{~Hz}, 12 \mathrm{H}), 1.35(\mathrm{~d}, J=6.8 \mathrm{~Hz}, 12 \mathrm{H}), 2.32-2.42(\mathrm{~m}, 2 \mathrm{H}), 3.06$ (sept, $J=6.8 \mathrm{~Hz}, 4 \mathrm{H}), 3.43(\mathrm{t}, J=5.9 \mathrm{~Hz}, 4 \mathrm{H}), 7.21(\mathrm{~d}, J=7.7 \mathrm{~Hz}, 4 \mathrm{H}), 7.33-7.40(\mathrm{~m}, 2 \mathrm{H})$

${ }^{13} \mathrm{C}$ NMR $\left(\mathrm{CDCl}_{3}\right) \delta 20.50,24.64,24.87,28.66,46.16,124.67,129.39,141.40,145.43$ 


\section{X-ray Crystallographic Analyses}

\section{Crystallographic characterization of 6DippCuCl and ${ }^{\mathrm{C}} \mathrm{IPrCuCl}$}

Crystals suitable for the X-ray structural determination were mounted on a Bruker SMART APEXII CCD diffractometer and irradiated with graphite monochromated Mo K $\alpha$ radiation $(\lambda=0.71073 \AA)$ for data collection. The data were processed using the APEX3 program suite and summarized in Table S4. All structures were solved by an intrinsic phasing method using the SHELXT program (ver. 2014/4-2014/5)23. Refinement on $F^{2}$ was carried out using full-matrix least-squares with the SHELXL ${ }^{24}$ and expanded using Fourier techniques. All nonhydrogen atoms, except those of disordered solvents, were refined using anisotropic thermal parameters. Hydrogen atoms were assigned to idealized geometric positions and included in structure factor calculations. The SHELX was interfaced with SHELXLE GUI for most of the refinement $\operatorname{steps}^{25}$. In the final stages of the refinement, analysis of the data with the TWINROTMAT routine in PLATON ${ }^{26}$ indicated the presence of a minor twin component rotated by use of a twin law of

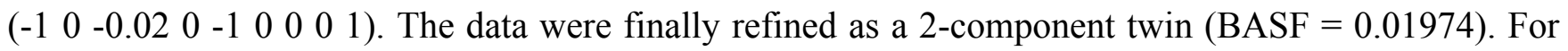
${ }^{\mathrm{C}} \mathrm{IPrCuCl}$, this unit cell contained a copper complex, dichloromethane and $n$-hexane in the ratio of 4:1:1. However, they could not be modelled satisfactorily and were therefore removed from the electron density map using the SQEEZE ${ }^{27}$ command in PLATON.

\section{Crystallographic characterization of ${ }^{\mathrm{Cl}} \mathrm{IMesCuCl}$}

Crystals suitable for the X-ray structural determination were mounted on a Rigaku XtaLAB Synergy DW system and irradiated with graphite monochromated Mo K $\alpha$ radiation $(\lambda=0.71073 \AA)$ for data collection. The data were processed using CrysAlisPro suite and summarized in Table S4. All structures were solved by an intrinsic phasing method using the SHELXT program (ver. 2018/3) (23 $^{2}$ Refinement on $F^{2}$ was carried out using full-matrix least-squares with the SHELXL ${ }^{24}$ and expanded using Fourier techniques. All nonhydrogen atoms, except those of disordered solvents, were refined using anisotropic thermal parameters. Hydrogen atoms were assigned to idealized geometric positions and included in structure factor calculations. The SHELX was interfaced with SHELXLE GUI for most of the refinement steps ${ }^{25}$. Flack parameter indicated $0.044(5)$ due to a highly symmetrical space group of Fdd2, therefore TWIN and BASF commands were conducted in the final refinement. $(\mathrm{BASF}=0.01994)$

All the pictures of the molecules were prepared using Pov-Ray $3.6^{28}$. 
Table S4. Crystal data and structure refinement for $6 \mathrm{DippCuCl},{ }^{\mathrm{Cl}} \mathrm{IPCuCl}$ and ${ }^{\mathrm{Cl}} \mathrm{IMesCuCl}$

\begin{tabular}{|c|c|c|c|c|c|c|}
\hline Identification code & \multicolumn{2}{|l|}{ 6DippCuCl } & \multicolumn{2}{|l|}{${ }^{\mathrm{Cl}} \mathrm{IPrCuCl}$} & \multicolumn{2}{|l|}{${ }^{\mathrm{Cl}} \mathrm{IMesCuCl}$} \\
\hline CCDC No. & \multicolumn{2}{|l|}{2085995} & \multicolumn{2}{|l|}{2085996} & \multicolumn{2}{|l|}{2120464} \\
\hline Empirical formula & \multicolumn{2}{|c|}{$\mathrm{C} 29 \mathrm{H} 42 \mathrm{Cl} 3 \mathrm{Cu} \mathrm{N} 2$} & \multicolumn{2}{|c|}{$\mathrm{C} 27 \mathrm{H} 34 \mathrm{Cl} 3 \mathrm{Cu}$ N2 } & \multicolumn{2}{|c|}{$\mathrm{C} 21 \mathrm{H} 22 \mathrm{Cl} 3 \mathrm{Cu} \mathrm{N} 2$} \\
\hline Formula weight & \multicolumn{2}{|l|}{588.53} & \multicolumn{2}{|l|}{556.45} & \multicolumn{2}{|l|}{472.29} \\
\hline Temperature & \multicolumn{2}{|l|}{$173(2) \mathrm{K}$} & \multicolumn{2}{|l|}{$173(2) \mathrm{K}$} & \multicolumn{2}{|l|}{$100(2) \mathrm{K}$} \\
\hline Wavelength & \multicolumn{2}{|l|}{$0.71073 \AA$} & \multicolumn{2}{|l|}{$0.71073 \AA$} & \multicolumn{2}{|l|}{$0.71073 \AA$} \\
\hline Crystal system & \multicolumn{2}{|l|}{ Monoclinic } & \multicolumn{2}{|l|}{ Monoclinic } & \multicolumn{2}{|l|}{ Orthorhombic } \\
\hline Space group & \multicolumn{2}{|l|}{$\mathrm{P} 2{ }_{1} / \mathrm{c}$} & \multicolumn{2}{|l|}{$\mathrm{C} 2 / \mathrm{c}$} & \multicolumn{2}{|l|}{ Fdd 2} \\
\hline \multirow{3}{*}{ Unit cell dimensions } & $\mathrm{a}=15.61(2) \AA$ & $\alpha=90^{\circ}$. & $\mathrm{a}=20.87(2) \AA$ & $\alpha=90^{\circ}$. & $\mathrm{a}=28.9374(6) \AA$ & $\alpha=90^{\circ}$ \\
\hline & $\mathrm{b}=8.347(7) \AA$ & $\beta=90.83(2)^{\circ}$. & $\mathrm{b}=14.172(15) \AA$ & $\beta=94.79(2)$ & $\mathrm{b}=14.2845(3) \AA$ & $\beta=90^{\circ}$. \\
\hline & $\mathrm{c}=23.22(2) \AA$ & $\gamma=90^{\circ}$. & $\mathrm{c}=42.07(4) \AA$ & $\gamma=90^{\circ}$. & $\mathrm{c}=10.6528(2) \AA$ & $\gamma=90^{\circ}$. \\
\hline Volume & \multicolumn{2}{|l|}{$3026(4) \AA^{3}$} & \multicolumn{2}{|l|}{$12397(22) \AA^{3}$} & \multicolumn{2}{|l|}{$4403.40(15) \AA^{3}$} \\
\hline $\mathrm{Z}$ & \multicolumn{2}{|l|}{4} & \multicolumn{2}{|l|}{16} & \multicolumn{2}{|l|}{8} \\
\hline Density (calculated) & $1.292 \mathrm{Mg} / \mathrm{m}^{3}$ & & $1.193 \mathrm{Mg} / \mathrm{m}^{3}$ & & $1.425 \mathrm{Mg} / \mathrm{m}^{3}$ & \\
\hline Absorption coefficient & $1.006 \mathrm{~mm}^{-1}$ & & $0.979 \mathrm{~mm}^{-1}$ & & $1.364 \mathrm{~mm}^{-1}$ & \\
\hline $\mathrm{F}(000)$ & 1240 & & 4640 & & 1936 & \\
\hline Crystal size & $0.297 \times 0.233 \times$ & $106 \mathrm{~mm} 3$ & $0.135 \times 0.120 \times 0$. & $38 \mathrm{~mm} 3$ & $0.100 \times 0.080 \times 0$ & $50 \mathrm{~mm} 3$ \\
\hline $\begin{array}{l}\text { Theta range for } \\
\text { data collection }\end{array}$ & 2.171 to 28.013 & & 1.739 to $25.306^{\circ}$. & & 2.487 to $31.111^{\circ}$ & \\
\hline Index ranges & $\begin{array}{l}-20<=\mathrm{h}<=20 \\
-10<=\mathrm{k}<=10 \\
-30<=\mathrm{l}<=30\end{array}$ & & $\begin{array}{l}-24<=\mathrm{h}<=24 \\
-16<=\mathrm{k}<=17 \\
-50<=\mathrm{l}<=50\end{array}$ & & $\begin{array}{l}-39<=\mathrm{h}<=38 \\
-20<=\mathrm{k}<=19 \\
-15<=1<=15\end{array}$ & \\
\hline Reflections collected & 35935 & & 59858 & & 25350 & \\
\hline Independent reflections & $7242[\mathrm{R}(\mathrm{int})=0$ & 283] & $11201[\mathrm{R}$ (int) $=0$ & 834] & $3107[\mathrm{R}($ int $)=0.0$ & 24] \\
\hline $\begin{array}{l}\text { Completeness to theta } \\
=26.000^{\circ}\end{array}$ & $100.00 \%$ & & $99.2 \%$ & & $100.00 \%$ & \\
\hline Refinement method & Full-matrix leas & quares on $\mathrm{F} 2$ & Full-matrix least-s & uares on F2 & Full-matrix least- & uares on F2 \\
\hline Data / restraints / parameters & $7242 / 30 / 353$ & & $11201 / 0 / 611$ & & $3107 / 1 / 128$ & \\
\hline Goodness-of-fit on F2 & 1.074 & & 1.066 & & 1.083 & \\
\hline Final $\mathrm{R}$ indices & $\mathrm{R}_{1}=0.0385$ & & $\mathrm{R}_{1}=0.0588$ & & $\mathrm{R}_{1}=0.0220$ & \\
\hline$[\mathrm{I}>2 \operatorname{sigma}(\mathrm{I})]$ & $\mathrm{wR}_{2}=0.1121$ & & $\mathrm{wR}_{2}=0.1531$ & & $\mathrm{wR}_{2}=0.0529$ & \\
\hline $\mathrm{R}$ indices (all data) & $\mathrm{R}_{1}=0.0444, \mathrm{wF}$ & $=0.1163$ & $\mathrm{R}_{1}=0.0867, \mathrm{wR}_{2}$ & 0.1665 & $\mathrm{R}_{1}=0.0244, \mathrm{wR}_{2}$ & 0.0534 \\
\hline Extinction coefficient & $\mathrm{n} / \mathrm{a}$ & & $\mathrm{n} / \mathrm{a}$ & & $\mathrm{n} / \mathrm{a}$ & \\
\hline Largest diff. peak and hole & 0.607 and -0.35 & $\AA^{-3}$ & 1.752 and -0.756 & $\AA^{-3}$ & 0.370 and -0.185 & \\
\hline
\end{tabular}


Figure S3. Solid-state structure of $6 \mathrm{DippCuCl}$ with $50 \%$ probability ellipsoids. All hydrogen atoms and solvent molecules are omitted for clarity.

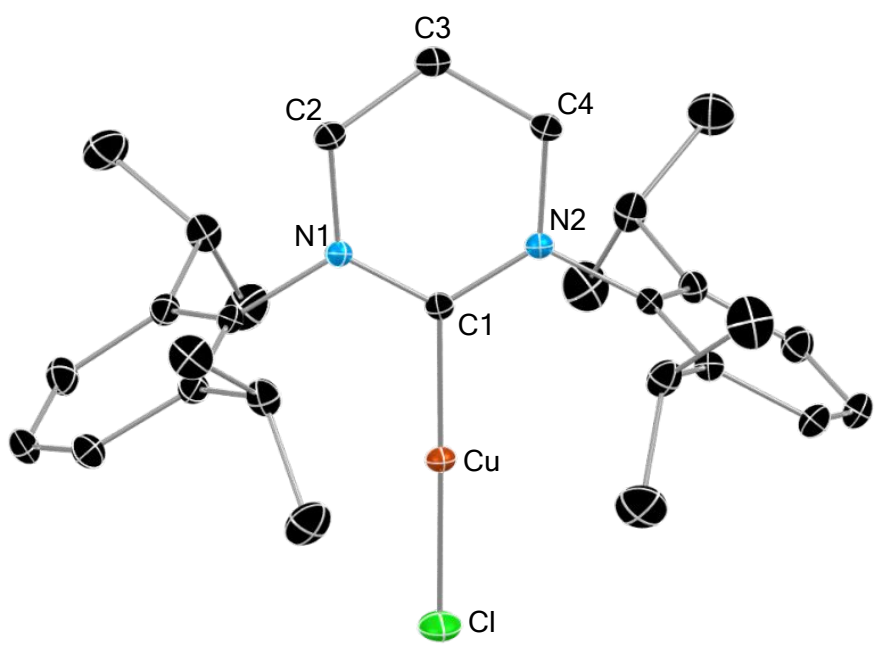

Figure S4. Solid-state structure of ${ }^{\mathrm{Cl}} \mathrm{IPrCuCl}$ with $50 \%$ probability ellipsoids. One of two independent molecules was shown. All hydrogen atoms are omitted for clarity.

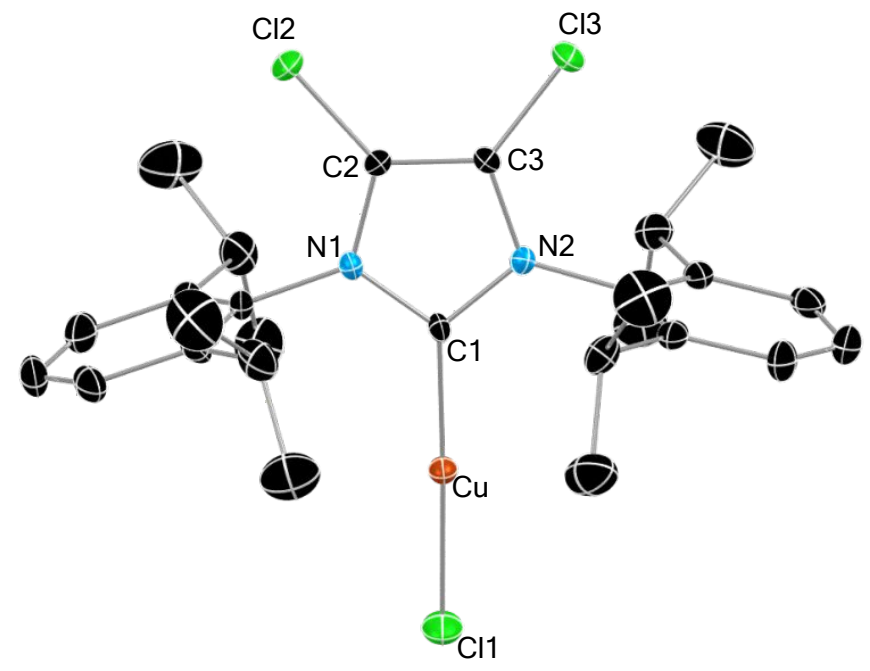

Figure S5. Solid-state structure of ${ }^{\mathrm{Cl}} \mathrm{IMesCuCl}$ with $50 \%$ probability ellipsoids. All hydrogen atoms are omitted for clarity. *: generated by symmetrical operation.

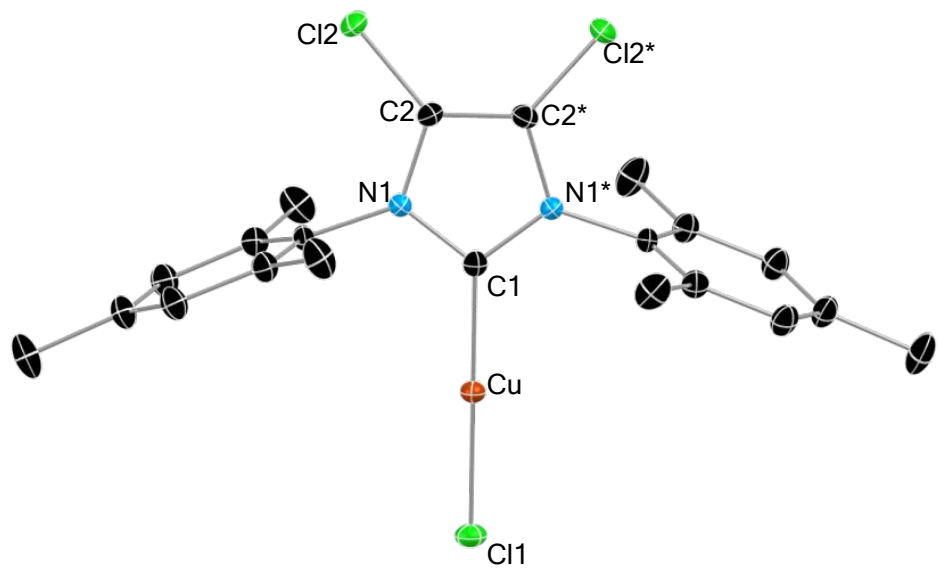




\section{References}

1. Landers, B.; Navarro, O. Microwave-Assisted Synthesis of (N-Heterocyclic carbene) $\mathrm{MCl}$ Complexes of Group 11 Metals. Eur. Inorg. Chem. 2012, 2980-2982.

2. Yoshida, H.; Hayashi, Y.; Ito, Y.; Takaki, K. Inverse regioselectivity in the silylstannylation of alkynes and allenes: copper-catalyzed three-component coupling with a silylborane and a tin alkoxide. Chem. Commun. 2015, 51, 9440-9442.

3. Fujihara, T.; Xu, T.; Semba, K.; Terao, J.; Tsuji, Y. Copper-Catalyzed Hydrocarboxylation of Alkynes Using Carbon Dioxide and Hydrosilanes. Angew. Chem. Int. Ed. 2011, 50, 523-527.

4. Yoshida, H.; Takemoto, Y.; Takaki, K. Direct Synthesis of Boron-Protected Alkenyl- and Alkylborons via Copper-Catalyzed Formal Hydroboration of Alkynes and Alkenes. Asian J. Org. Chem. 2014, 3, 1204-1209.

5. Becke, A. D. Density-functional exchange-energy approximation with correct asymptotic behavior. Phys. Rev. A 1988, 38, 3098-3100.

6. Becke, A. D. A new mixing of Hartree-Fock and local density-functional theories. J. Chem. Phys. 1993, 98, 1372-1377.

7. Lee, C.; Yang, W.; Parr, R. G. Development of the Colle-Salvetti correlation-energy formula into a functional of the electron density. Phys. Rev. B 1988, 37, 785-789.

8. Ditchfield, R.; Hehre, W. J.; Pople, J. A. Self-Consistent Molecular-Orbital Methods. IX. An Extended Gaussian-Type Basis for Molecular-Orbital Studies of Organic Molecules. J. Chem. Phys. 1971, 54, 724-728.

9. Parida, R.; Nambiar, S. R.; Reddy, G. N.; Giri, S. Designing aromatic heterocyclic superacids in terms of Brønsted and Lewis perspectives. Phys. Chem. Chem. Phys. 2020, 22, 1923-1931.

10. Frisch, M. J.; Trucks, G. W.; Schlegel, H. B.; Scuseria, G. E.; Robb, M. A.; Cheeseman, J. R.; Scalmani, G.; Barone, V.; Petersson, G. A.; Nakatsuji, H.; Li, X.; Caricato, M.; Marenich, A. V.; Bloino, J.; Janesko, B. G.; Gomperts, R.; Mennucci, B.; Hratchian, H. P.; Ortiz, J. V.; Izmaylov, A. F.; Sonnenberg, J. L.; Williams-Young, D.; Ding, F.; Lipparini, F.; Egidi, F.; Goings, J.; Peng, B.; Petrone, A.; Henderson, T.; Ranasinghe, D.; Zakrzewski, V. G.; Gao, J.; Rega, N.; Zheng, G.; Liang, W.; Hada, M.; Ehara, M.; Toyota, K.; Fukuda, R.; Hasegawa, J.; Ishida, M.; Nakajima, T.; Honda, Y.; Kitao, O.; Nakai, H.; Vreven, T.; Throssell, K.; Montgomery, J. A.; Jr.; Peralta, J. E.; Ogliaro, F.; Bearpark, M. J.; Heyd, J. J.; Brothers, E. N.; Kudin, K. N.; Staroverov, V. N.; Keith, T. A.; Kobayashi, R.; Normand, J.; Raghavachari, K.; Rendell, A. P.; Burant, J. C.; Iyengar, S. S.; Tomasi, J.; Cossi, M.; Millam, J. M.; Klene, M.; Adamo, C.; Cammi, R.; Ochterski, J. W.; Martin, R. L.; Morokuma, 
K.; Farkas, O.; Foresman, J. B.; Fox, D. J. Gaussian 16, Revision B.01; Gaussian, Inc.: Wallingford, CT, 2016.

11. Böhrer, H.; Trapp, N.; Himmel, D.; Schleep, M.; Krossing, I. From unsuccessful $\mathrm{H}_{2}$-activation with FLPs containing $\mathrm{B}(\text { Ohfip })_{3}$ to a systematic evaluation of the Lewis acidity of 33 Lewis acids based on fluoride, chloride, hydride and methyl ion affinities. Dalton Trans. 2015, 44, 7489-7499.

12. Erdmann, P.; Leitner, J.; Schwarz, J.; Greb, L. An Extensive Set of Accurate Fluoride Ion Affinities for $p$-Block Element Lewis Acids and Basic Design Principles for Strong Fluoride Ion Acceptors. ChemPhysChem 2020, 21, 987-994.

13. Falivene, L.; Credendino, R.; Poater, A.; Petta, A.; Serra, L.; Oliva, R.; Scarano, V.; Cavallo, L. "SambVca2 - A Web Tool for Analyzing Catalytic Pockets with Topographic Steric Maps" Organometallics 2016, 35, 2286-2293.

14. Falivene, L.; Cao, Z.; Petta, A.; Serra, L.; Poater, A.; Oliva, R.; Scarano, V.; Cavallo, L. Towards the Online Computer-aided Design of Catalytic Pokets. Nat. Chem. 2019, 11, 872-879.

15. Laitar, D. S.; Müller, P.; Sadighi, J. P. Efficient Homogeneous Catalysis in the Reduction of $\mathrm{CO}_{2}$ to CO. J. Am. Chem. Soc. 2005, 127, 17196-17197.

16. Drescher, W.; Borner, C.; Kleeberg, C. Stability and decomposition of copper(I) boryl complexes: [(IDipp)Cu-Bneop], [(IDipp*)Cu-Bneop] and copper clusters. New J. Chem. 2021, 45, 1495714964.

17. Gasperini, D.; Maggi, L.; Nolan, S. P. Gold(I)-Catalysed Cyclisation of Alkynoic Acids: Towards an Efficient and Eco-Friendly Synthesis of $\gamma-, \delta$ - and $\epsilon$-Lactones. Adv. Synth. Catal. 2016, 358, 38573862.

18. Chen, Y.; Shuai, B.; Mei, T. Regioselective Ni-Catalyzed Carboxylation of Allylic and Propargylic Alcohols with Carbon Dioxide. Org. Lett. 2017, 19, 2969-2972.

19. Kale, T. S.; Tovar, J. D. Regulation of peptide- $\pi$-peptide nanostructure bundling: the impact of 'cruciform' $\pi$-electron segments. Tetrahedron 2017, 72, 6084-6090.

20. Hitosugi, S.; Tanimoto, D.; Isobe, H. A Facile Chromatographic Method for Purification of Pinacol Boronic Esters. Chem. Lett. 2012, 41, 972-973.

21. Malysheva, Y. B.; Fedorov, A. Y. Negishi Cross-Coupling Reaction as a Route to Isocombretastatins. Synlett 2013, 24, 1772-1776.

22. Jordan, A. J.; Wyss, C. M.; Sadighi, J. P. Synthesis and Reactivity of New Copper(I) Hydride Dimers. Organometallics 2016, 35, 613-616.

23. Sheldrick, G. M. SHELXT - Integrated space-group and crystal-structure determination. Acta Crystallogr., Sect. A: Found. Adv. 2015, 71, 3-8.

24. Sheldrick, G. M. A short history of SHELX. Acta Crystallogr., Sect. A: Found. Crystallogr. 2008, A64, 112-122. 
25. Huebschle, C. B.; Sheldrick, G. M.; Dittrich, B. ShelXle: a Qt graphical user interface for SHELXL. J. Appl. Crystallogr. 2011, 44, 1281-1284.

26. Spek, A. L. Structure validation in chemical crystallography, Acta Cryst. 2009, D65, 148-155.

27. Spek, A. L. PLATON SQUEEZE: a tool for the calculation of the disordered solvent contribution to the calculated structure factors Acta Crystallogr. Sect. C, 2015, C71, 9-18.

28. Cason, C. Pov-Ray 3.6.2; Persistence of Vision Raytracer. 2009. 


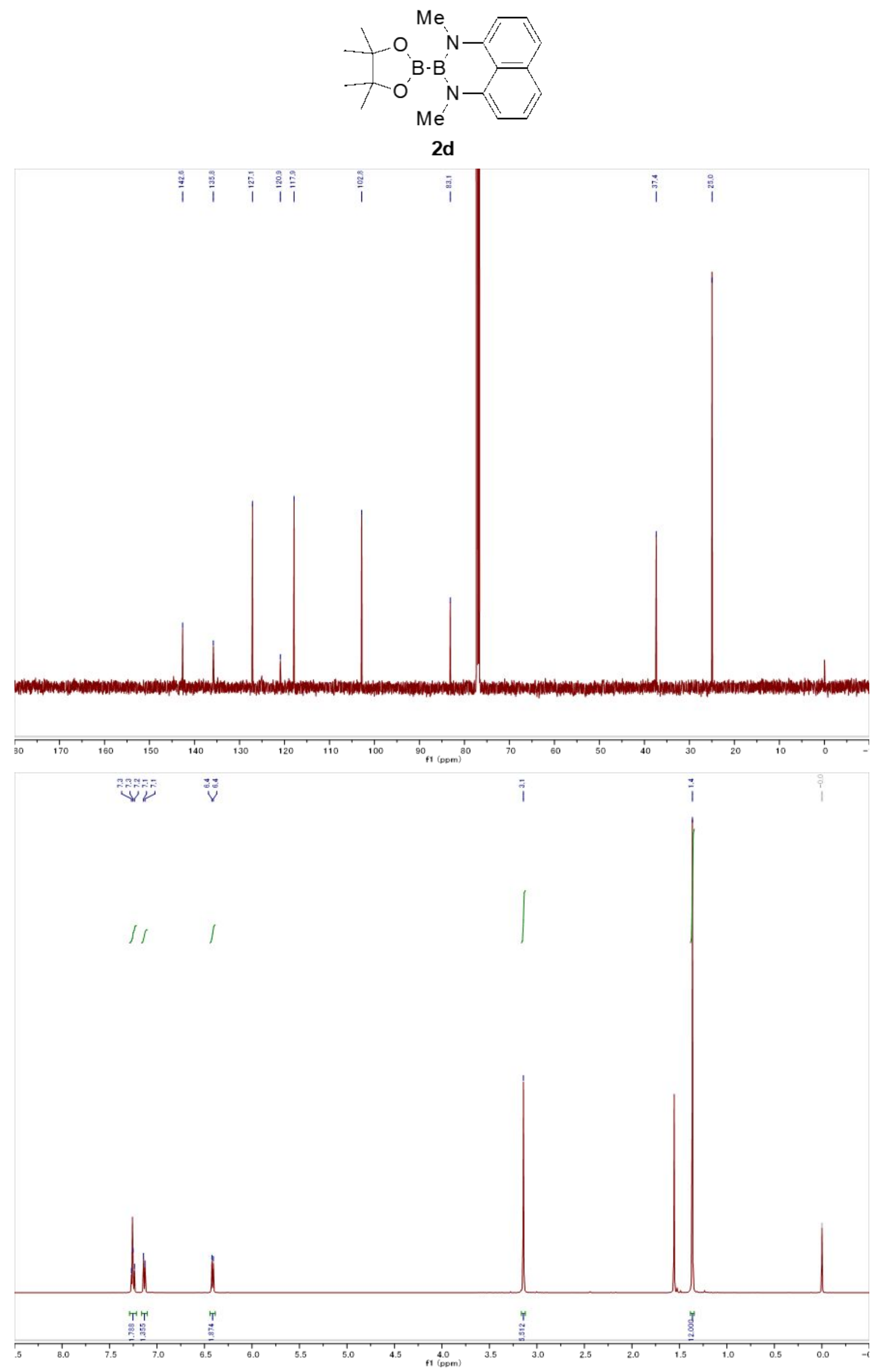




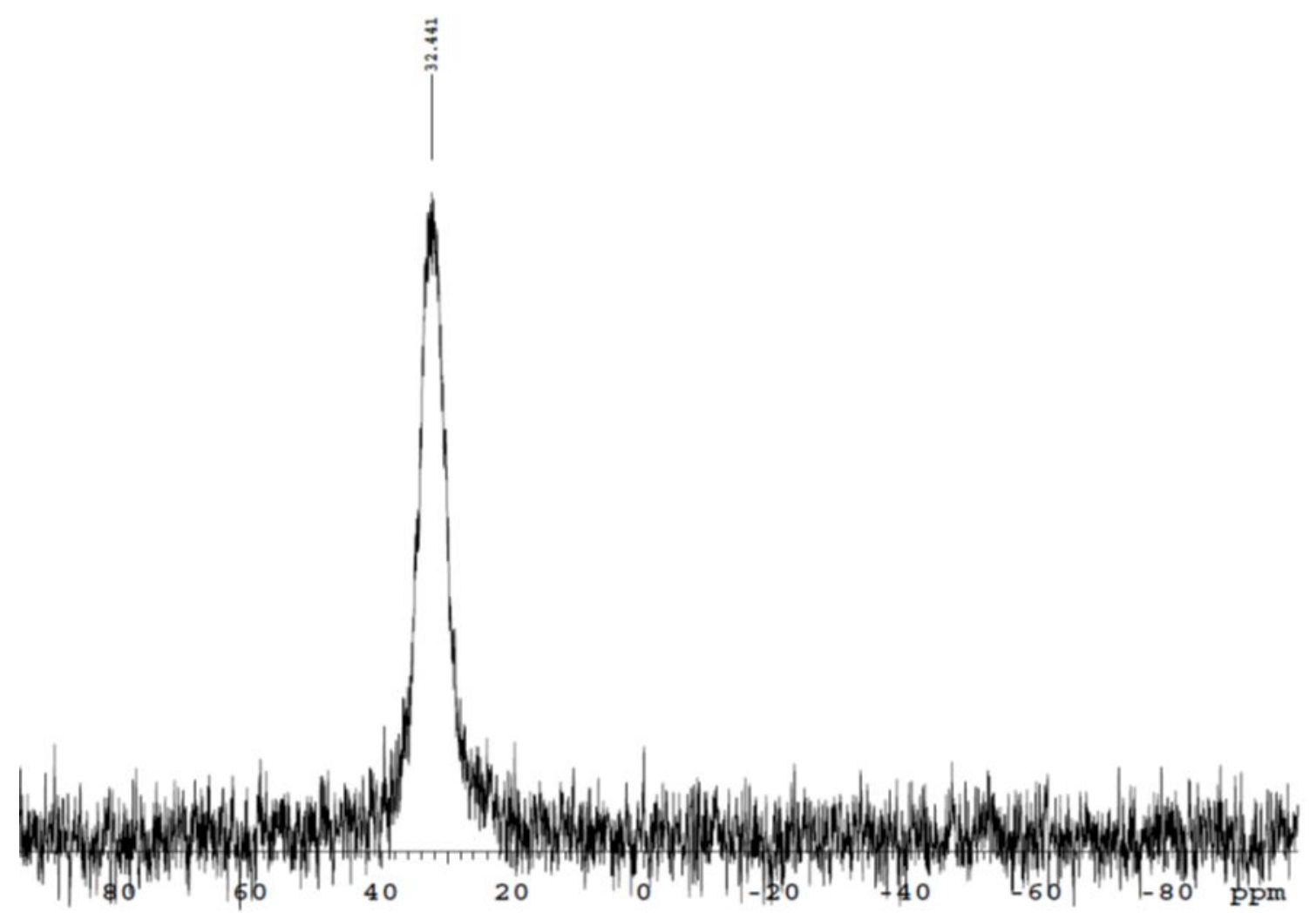




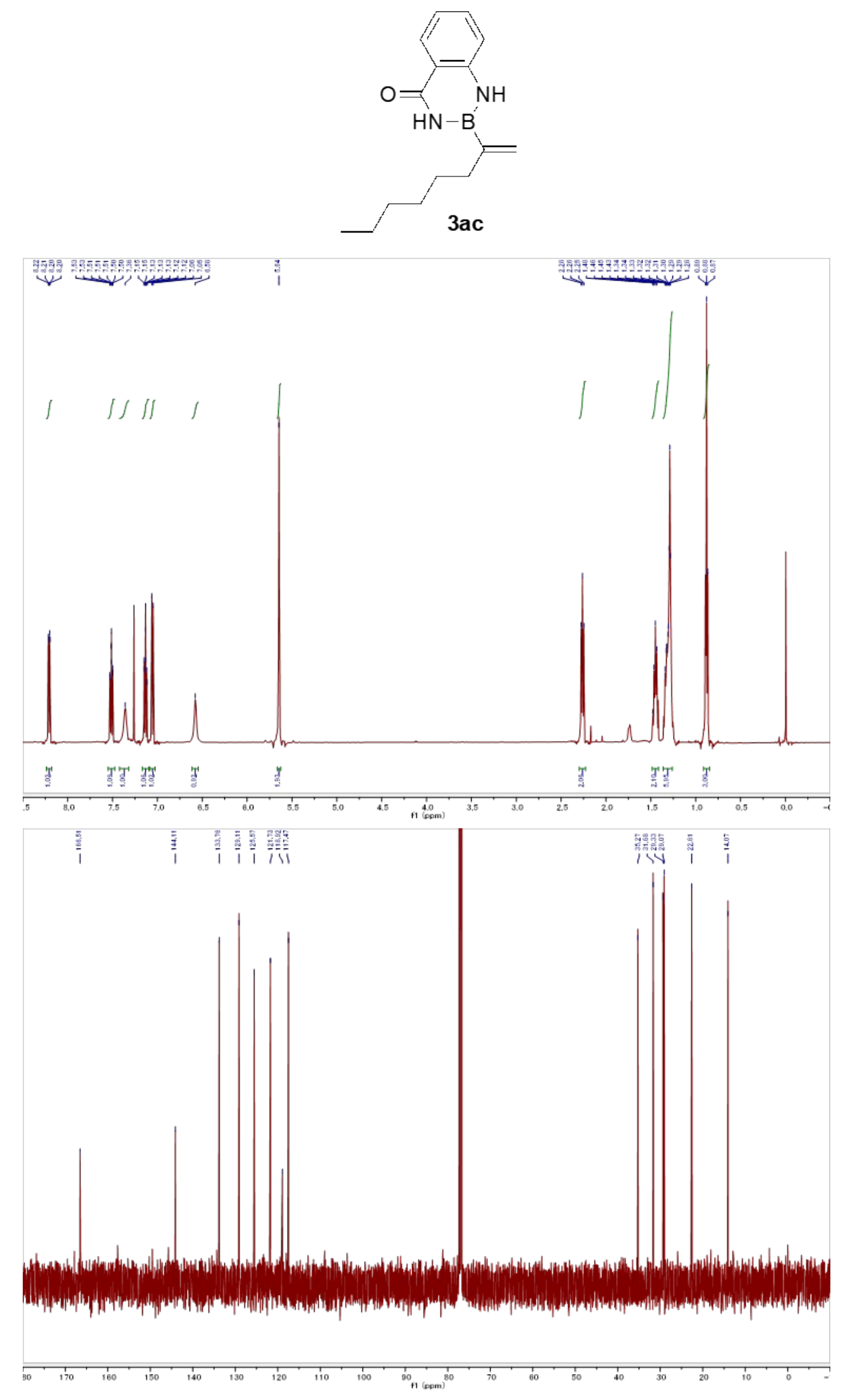




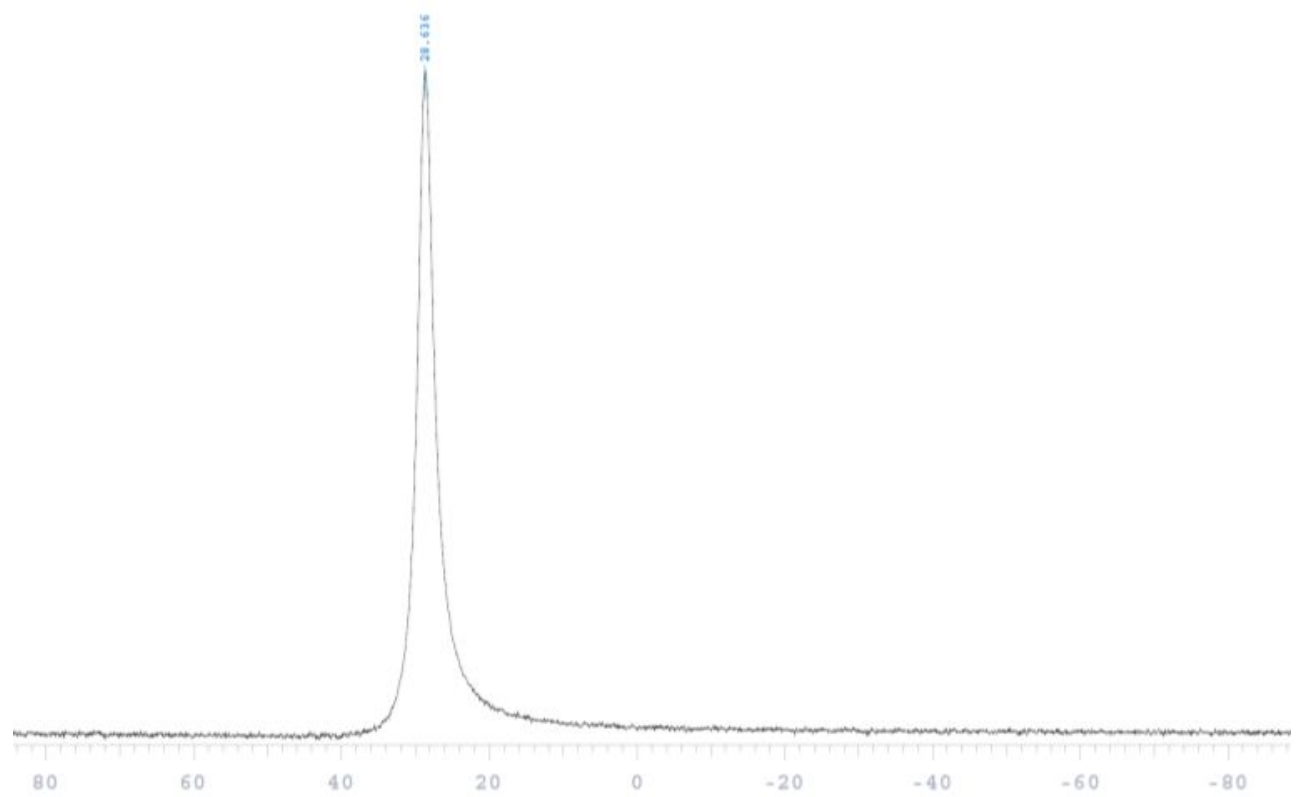



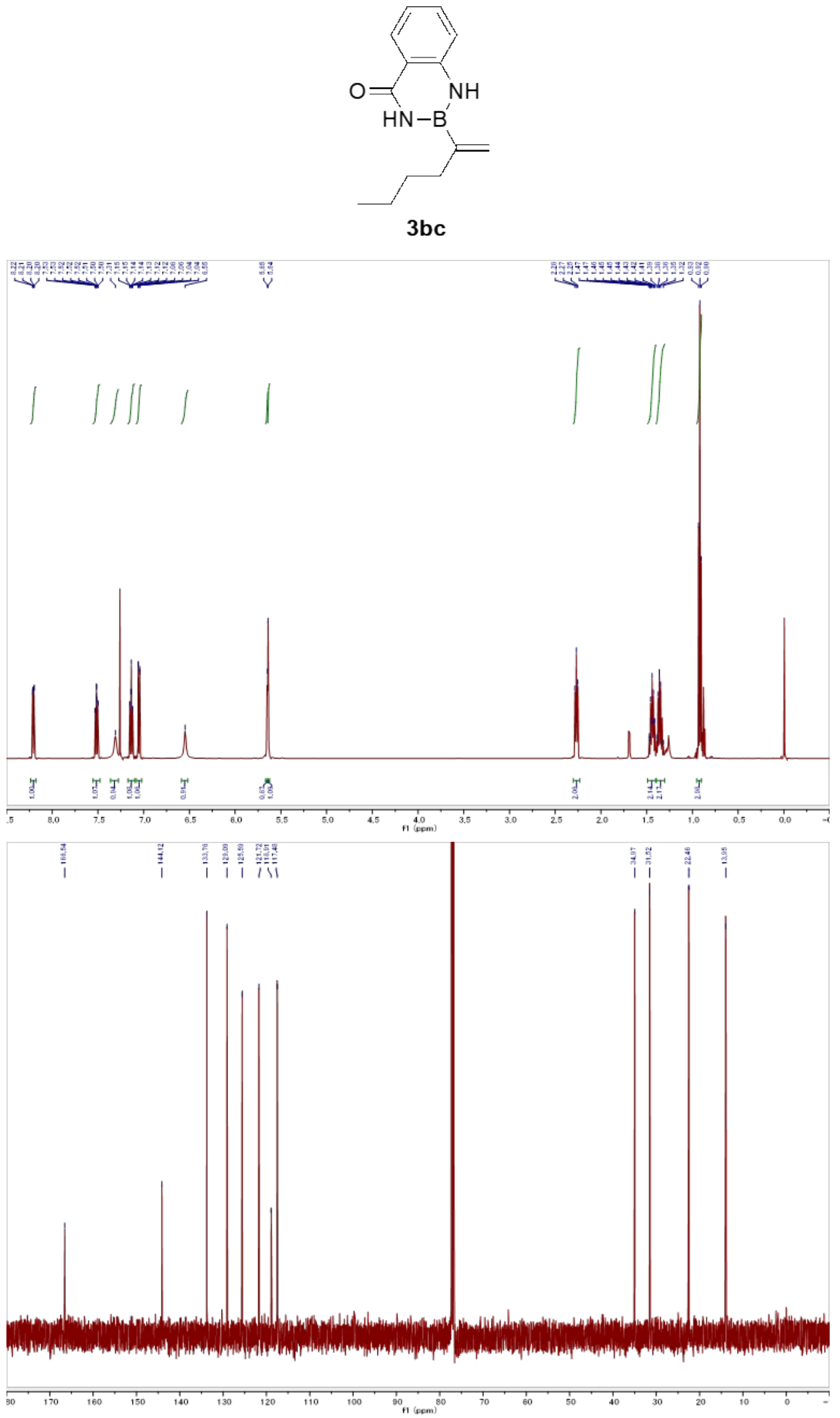


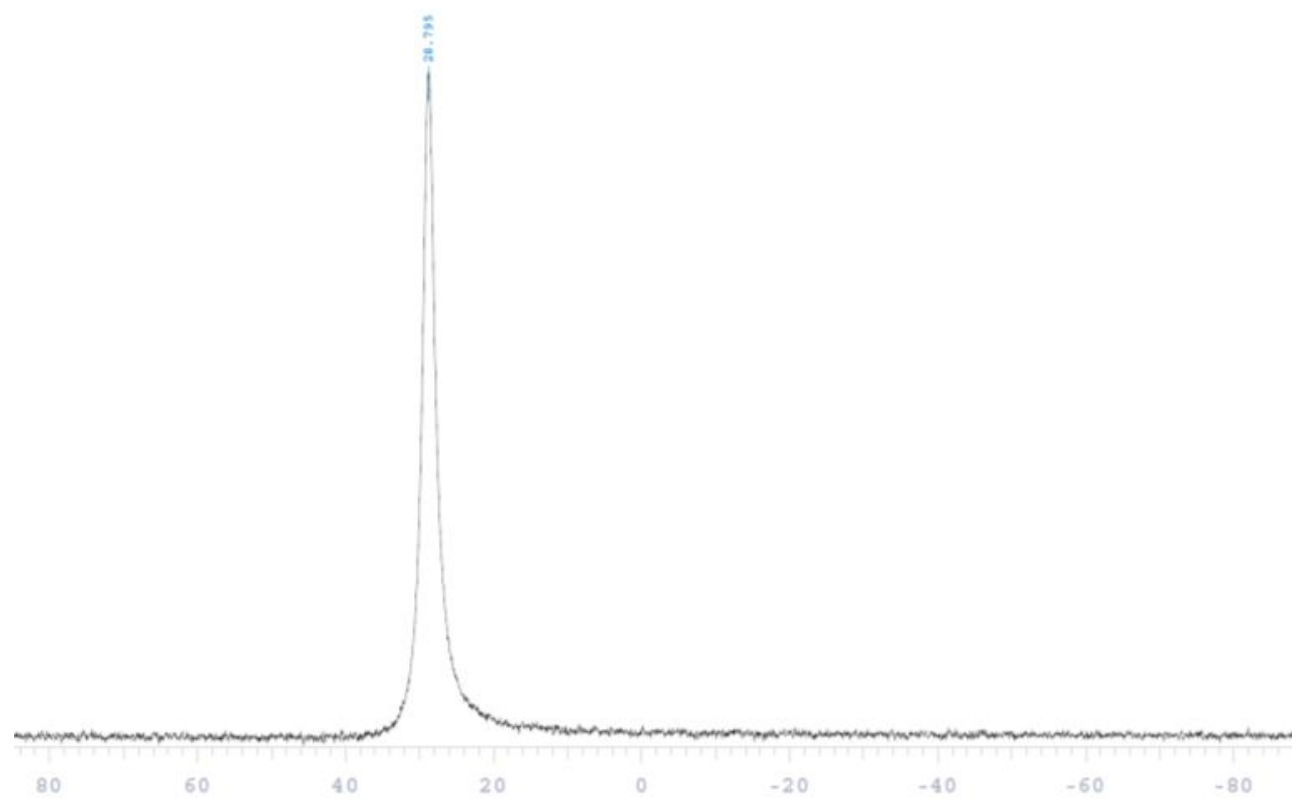




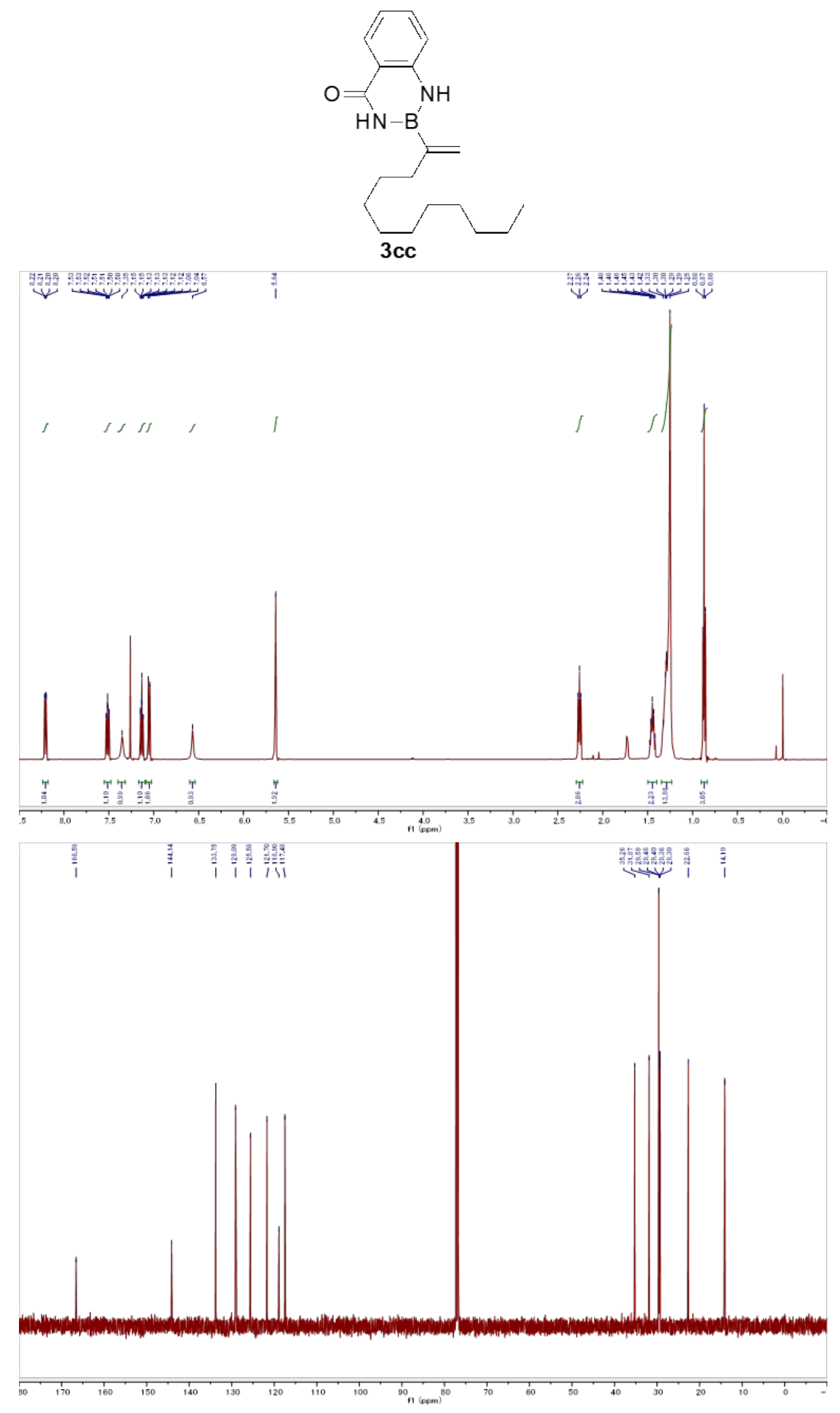




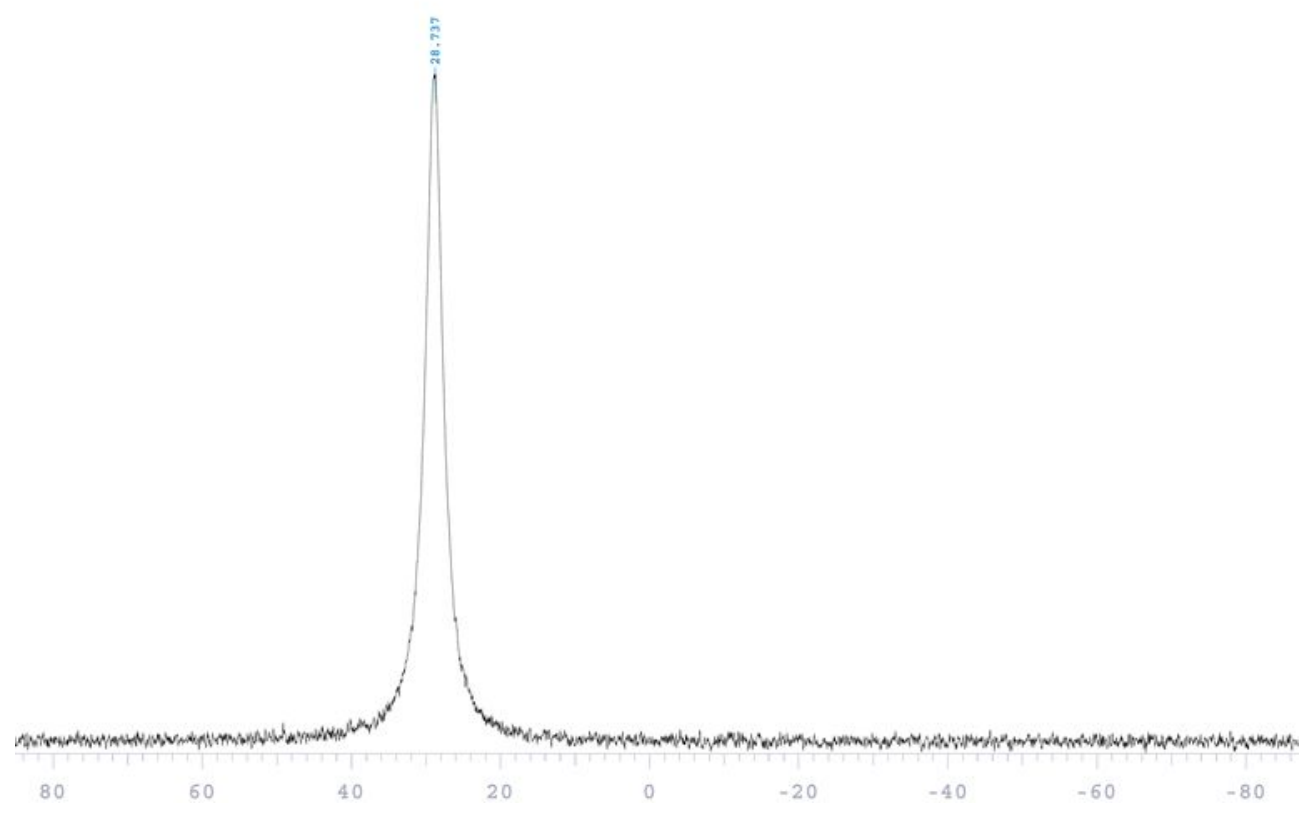



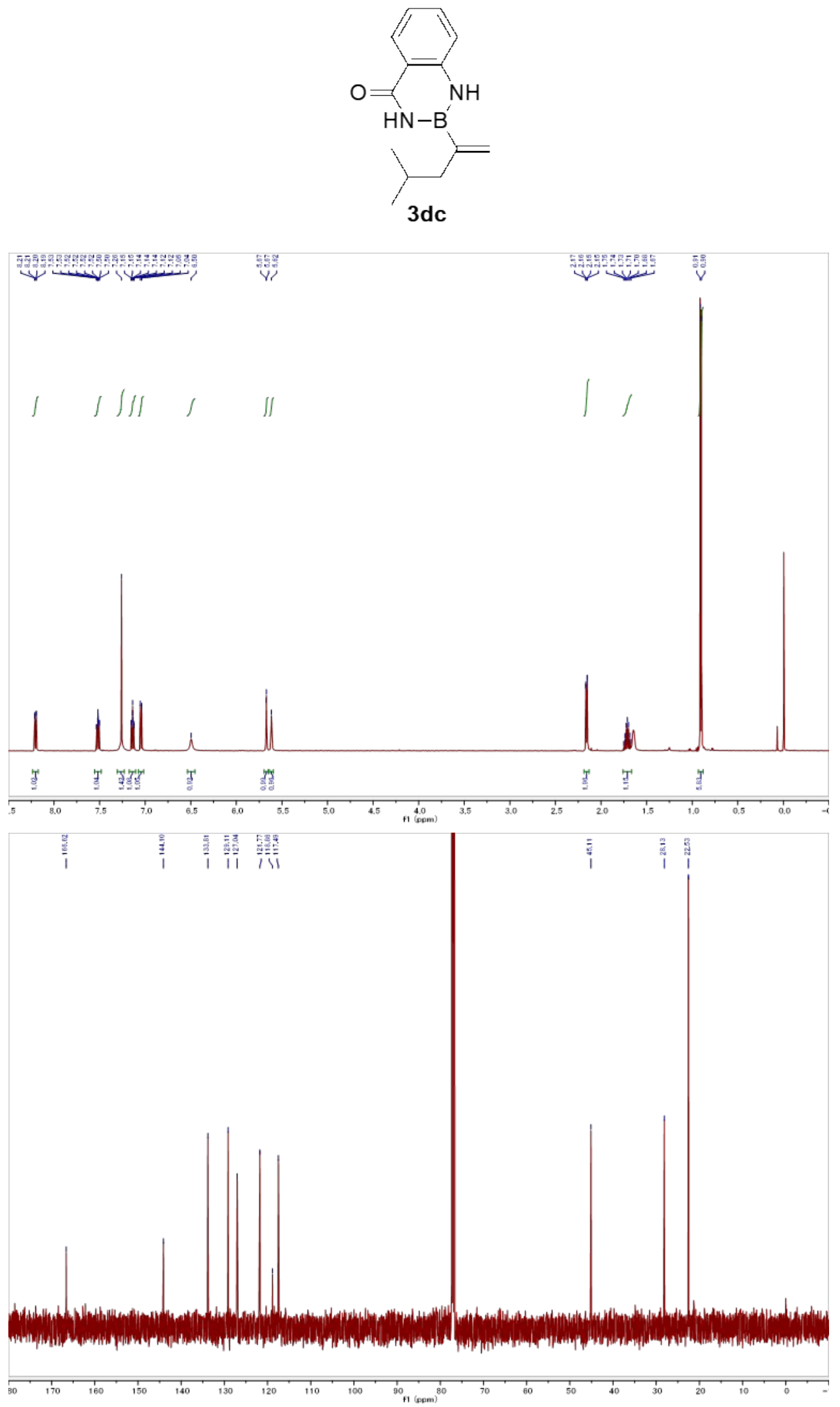


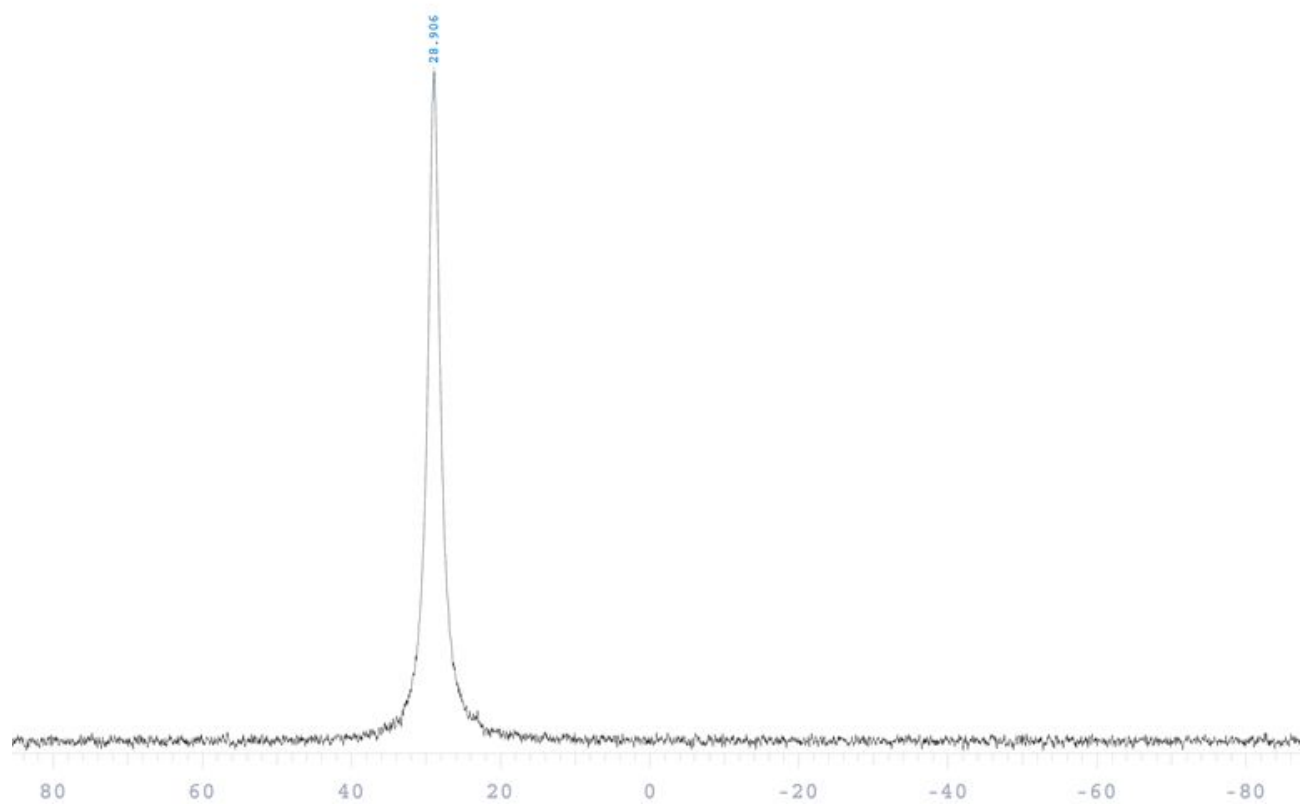



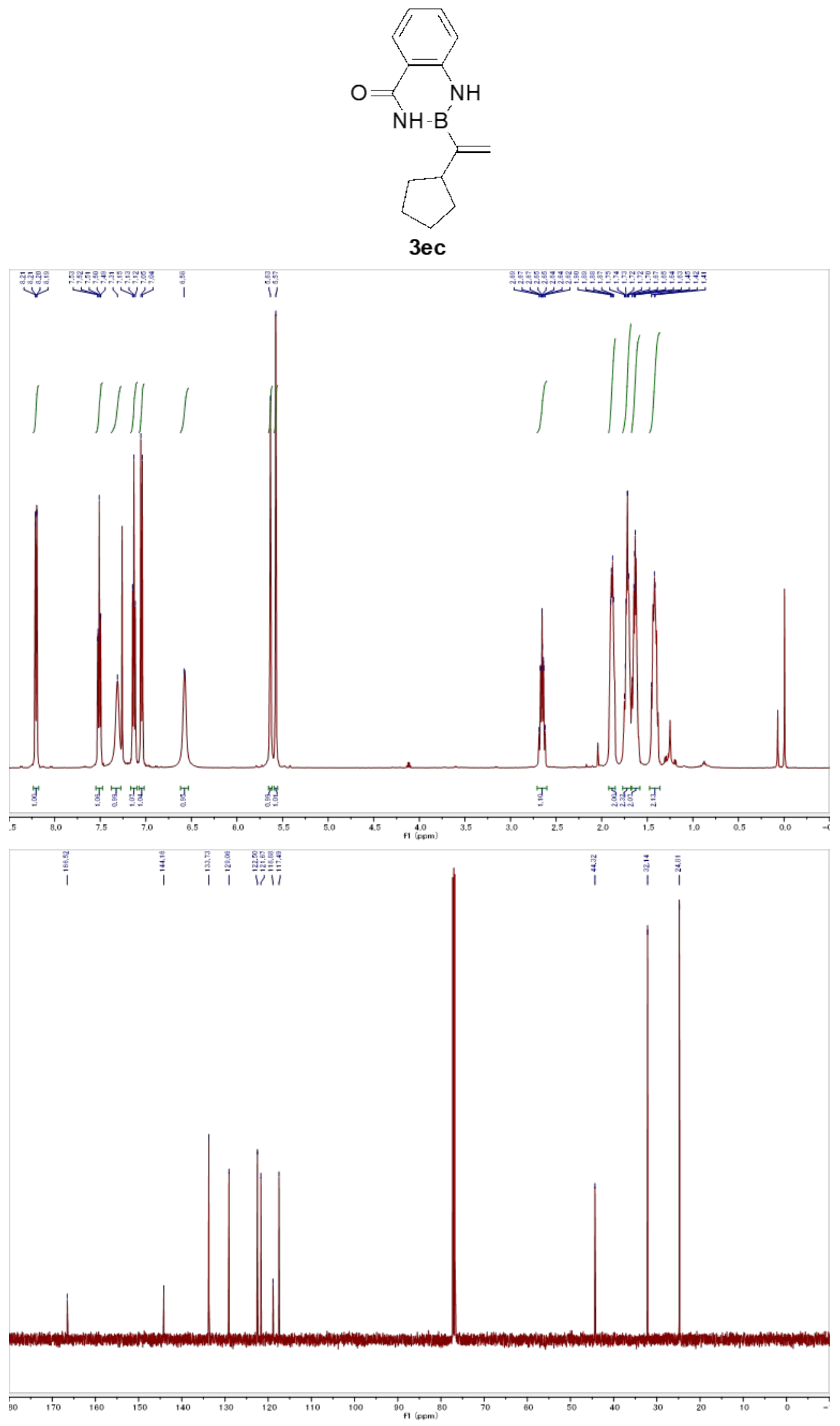


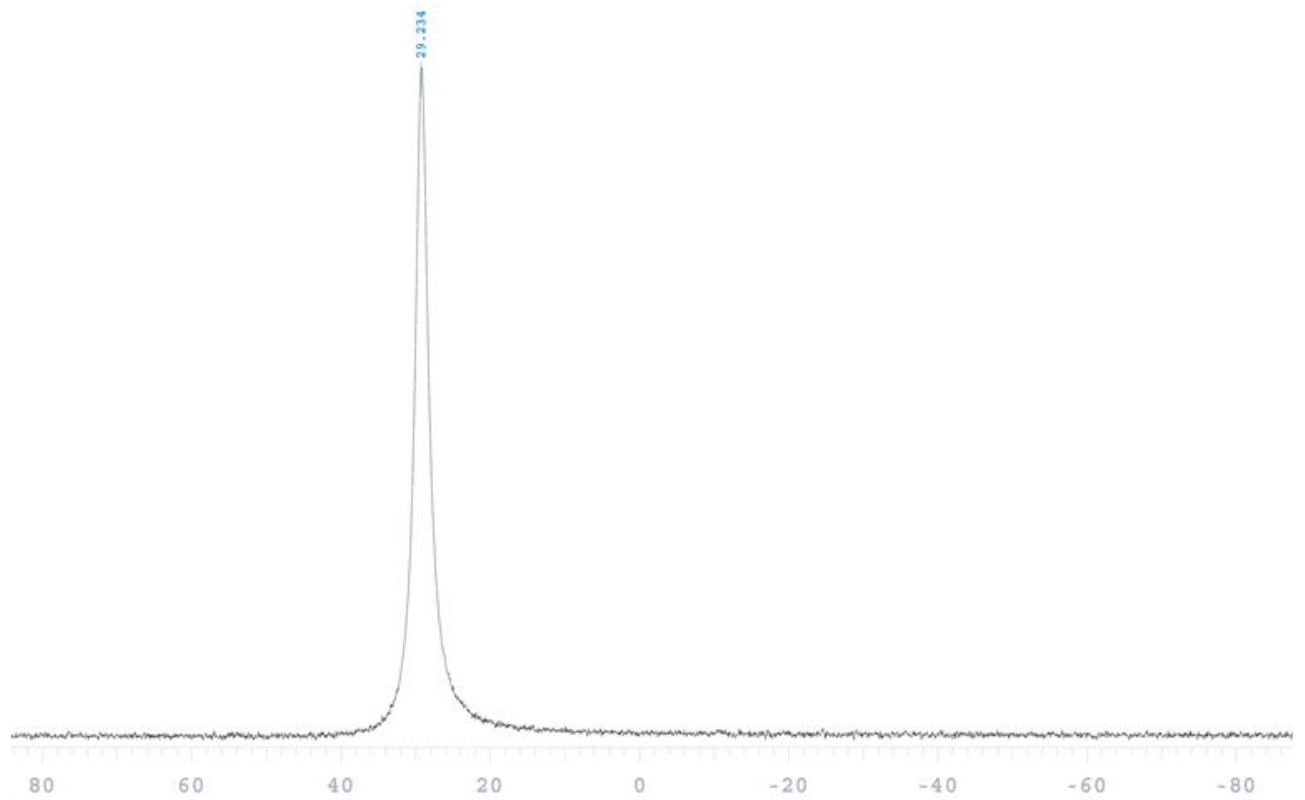




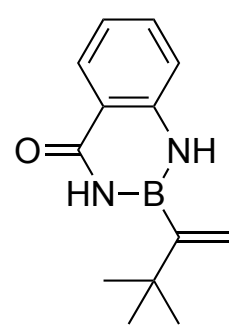

$3 f c$
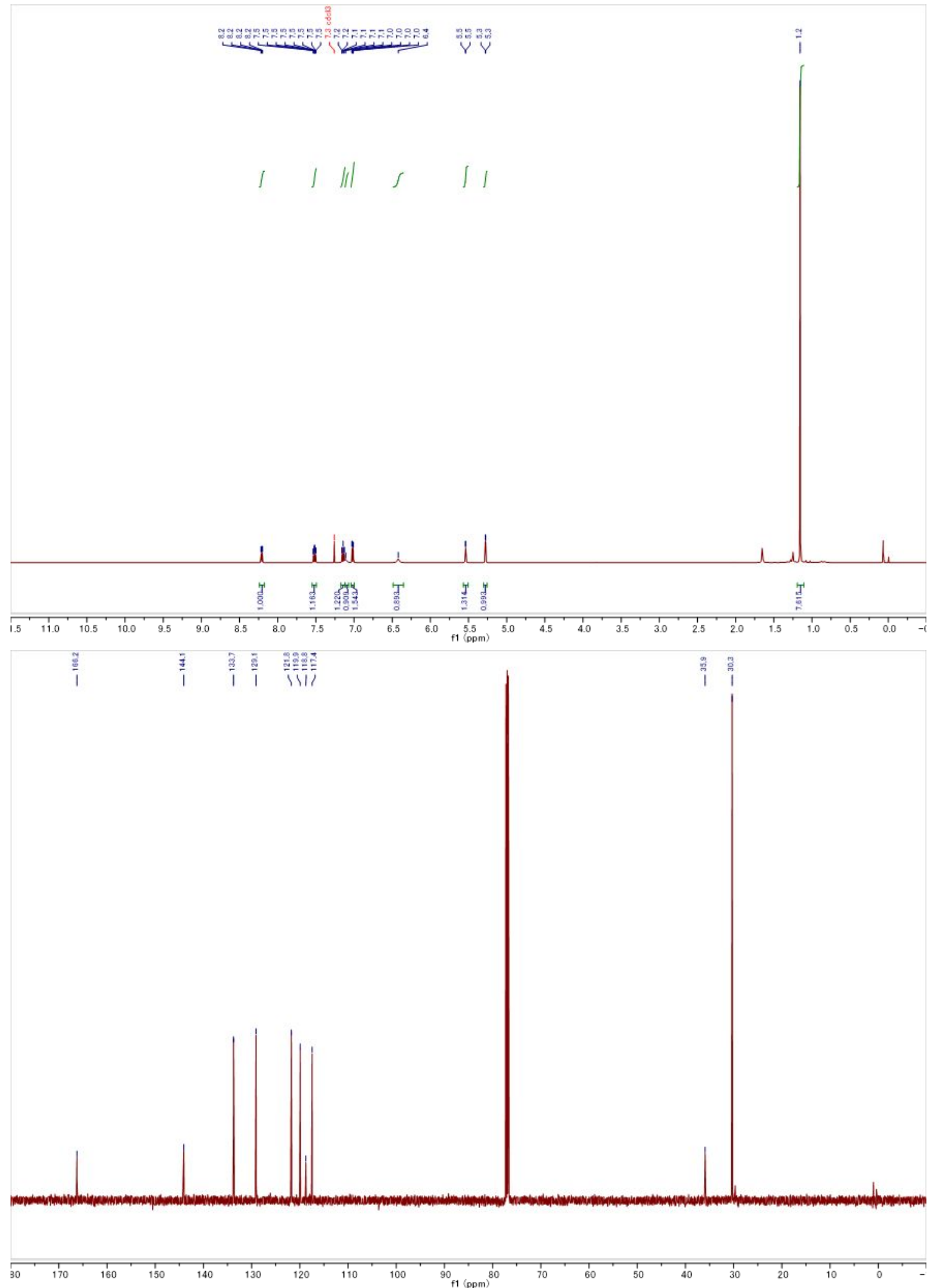


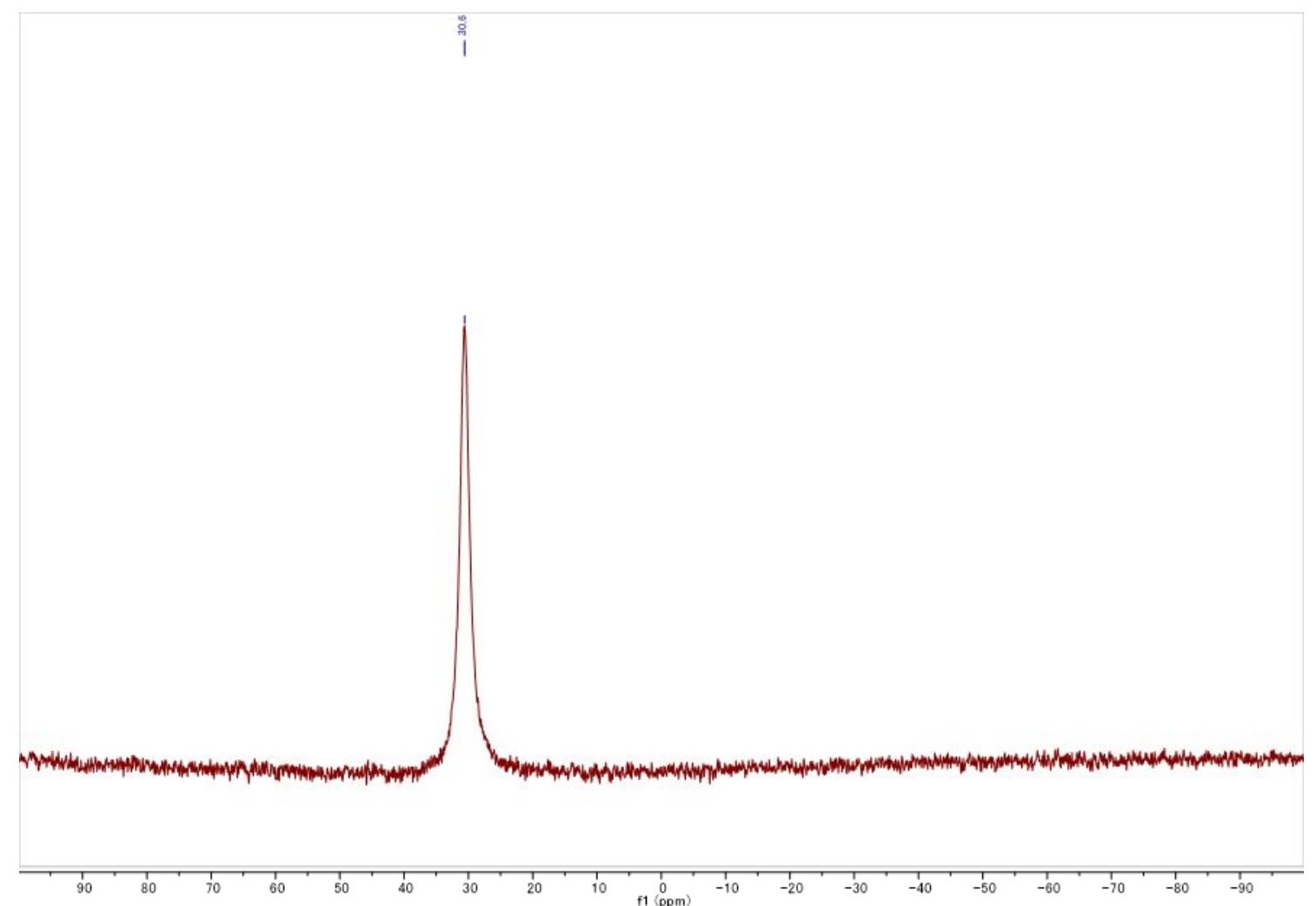



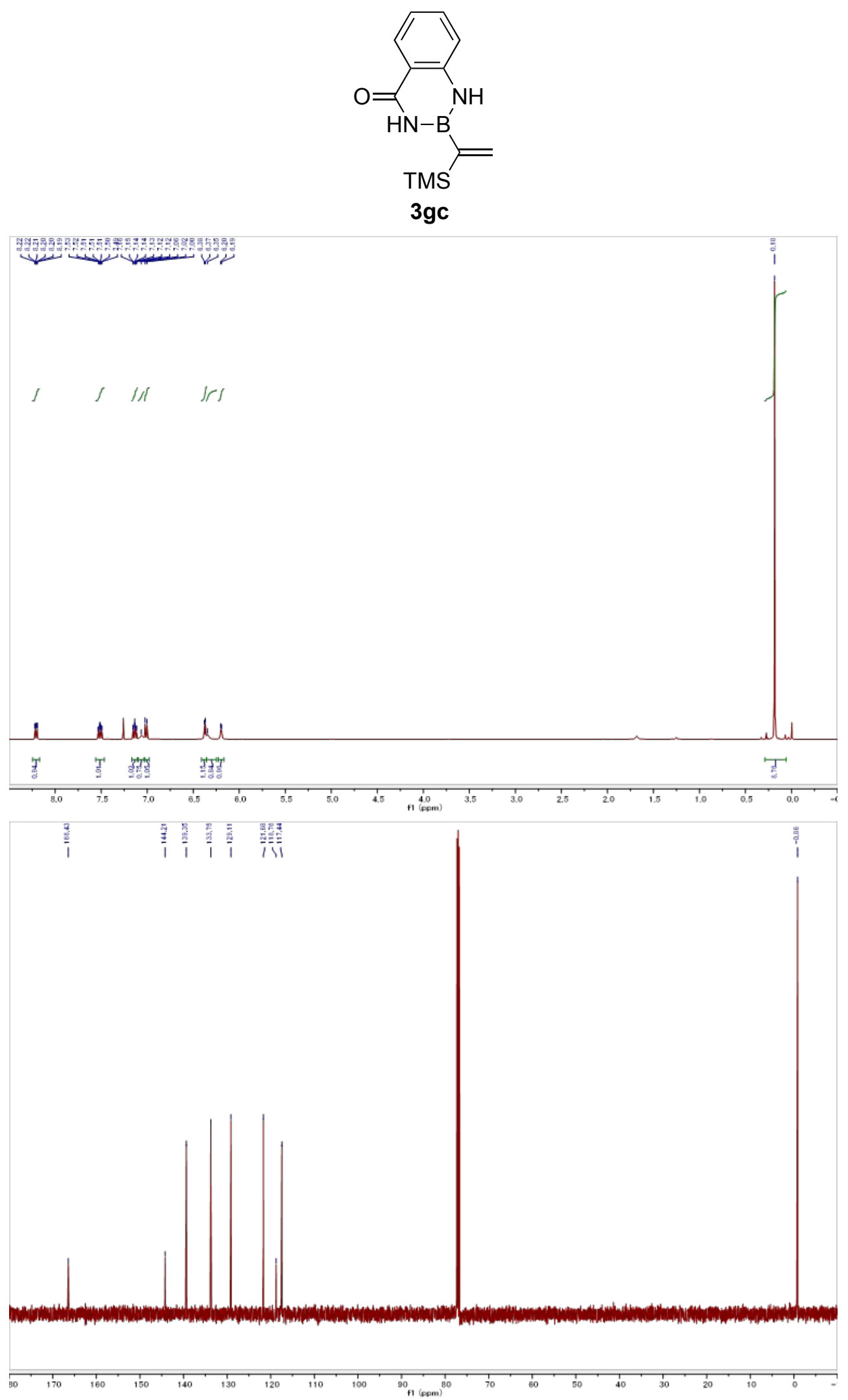


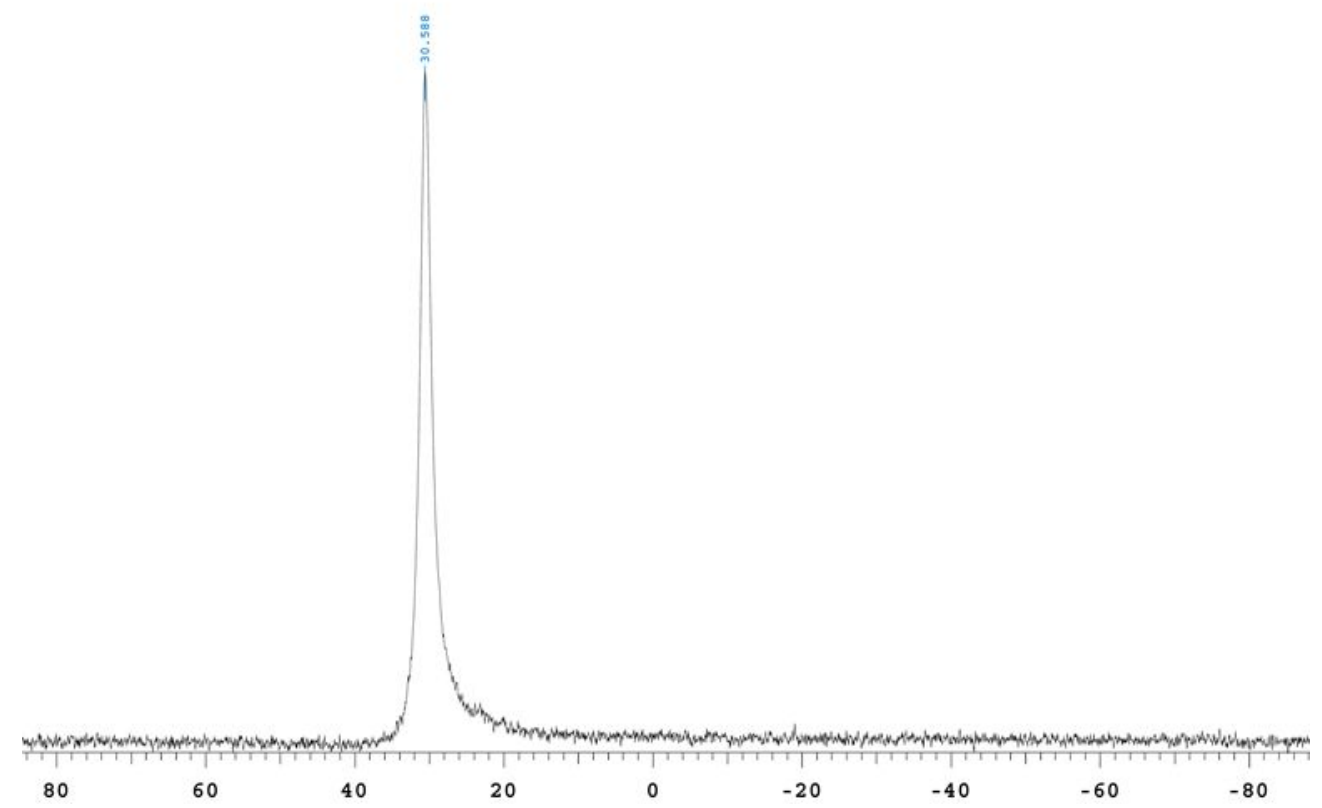



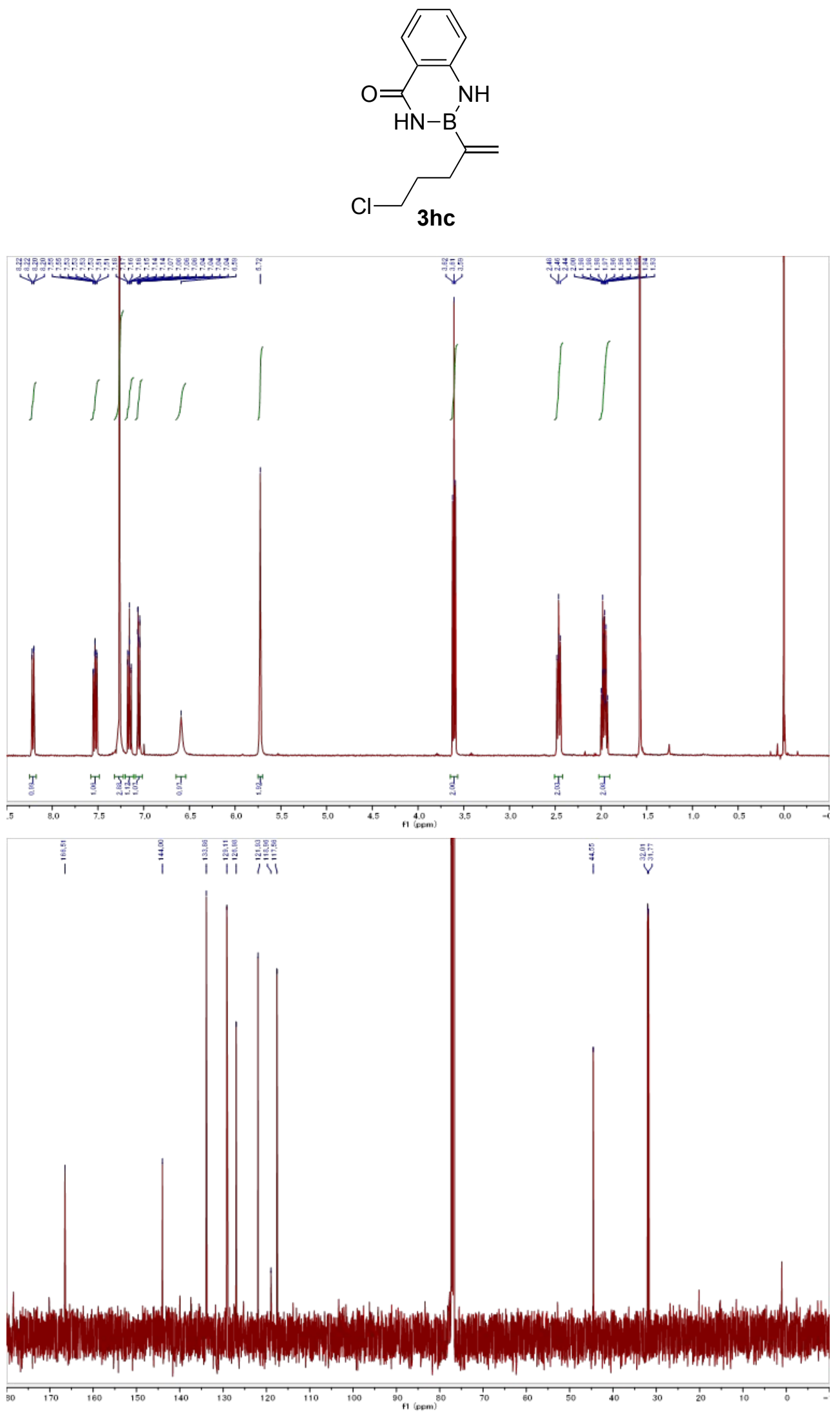


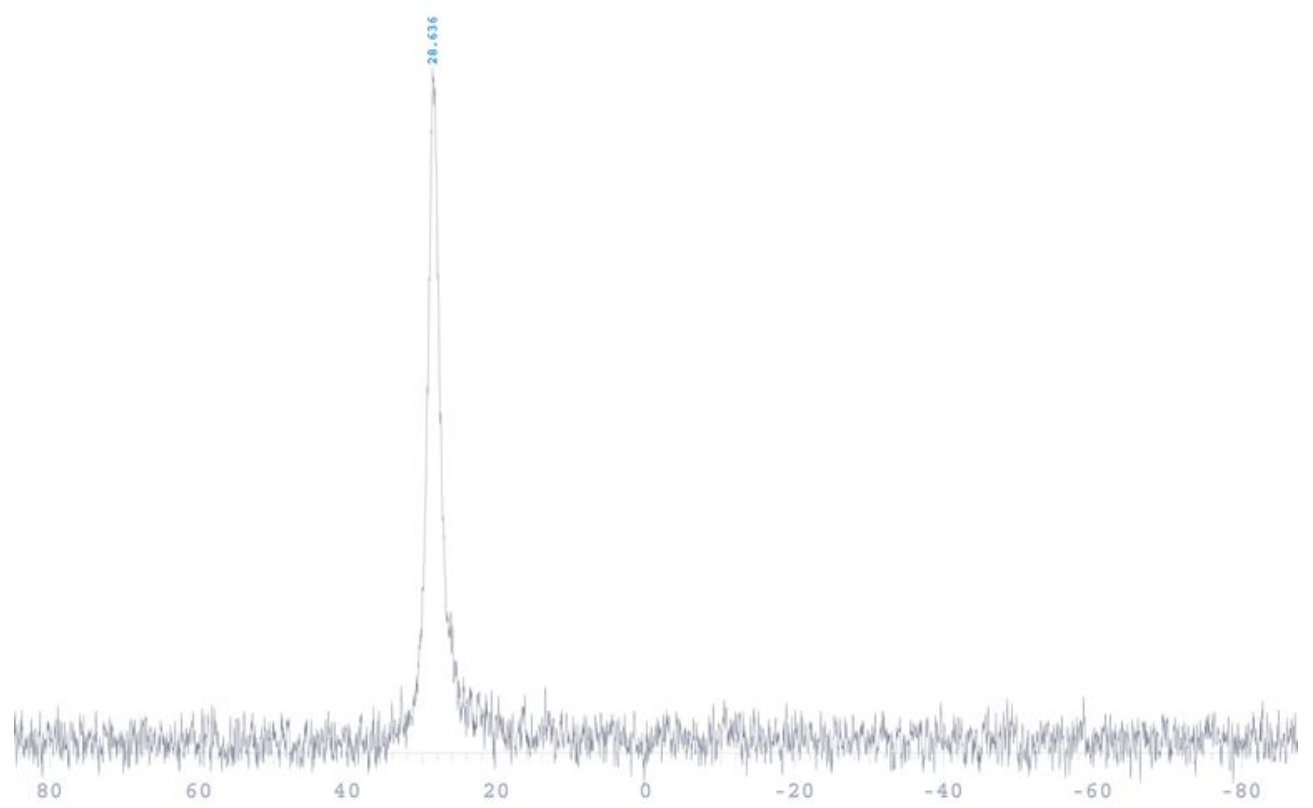



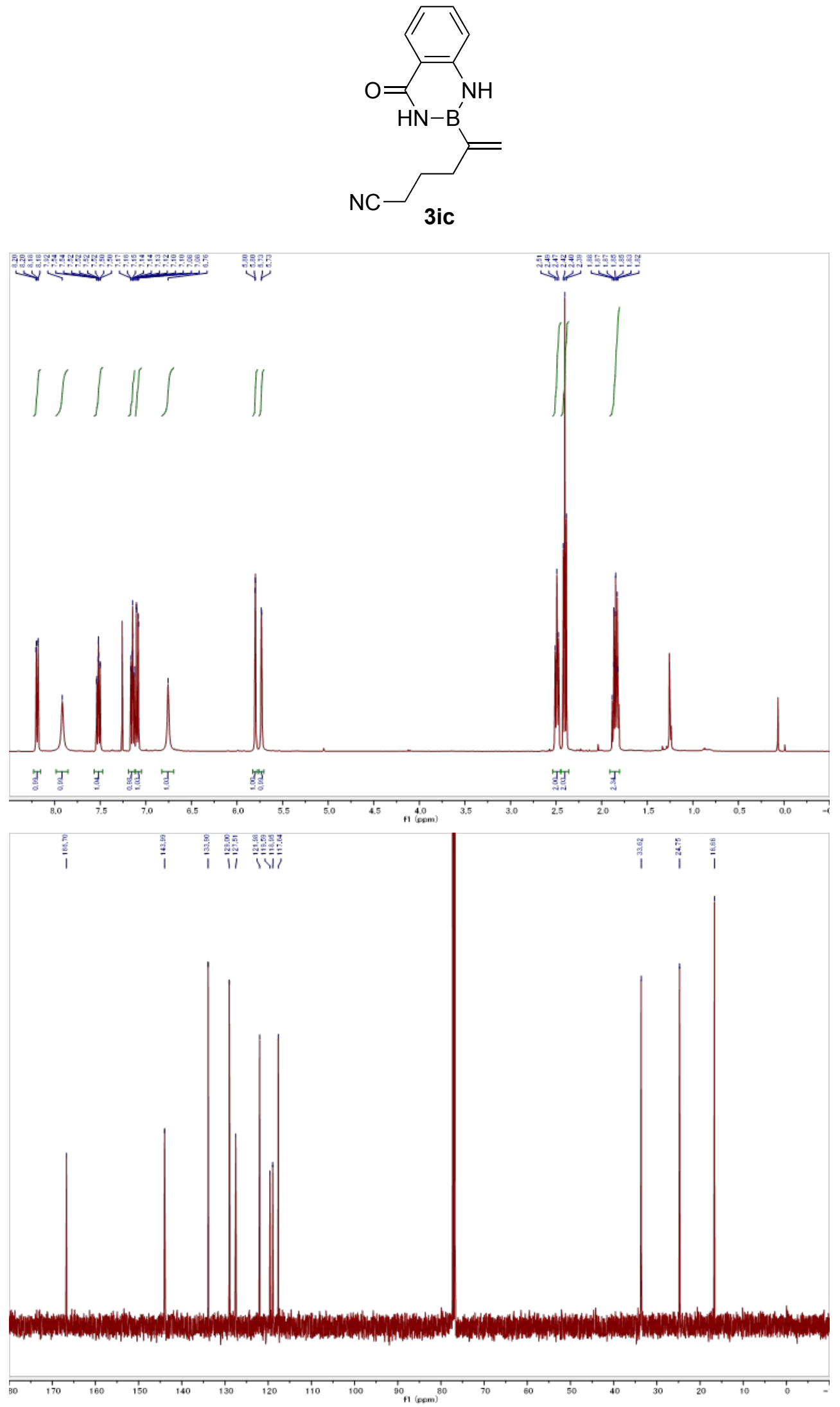


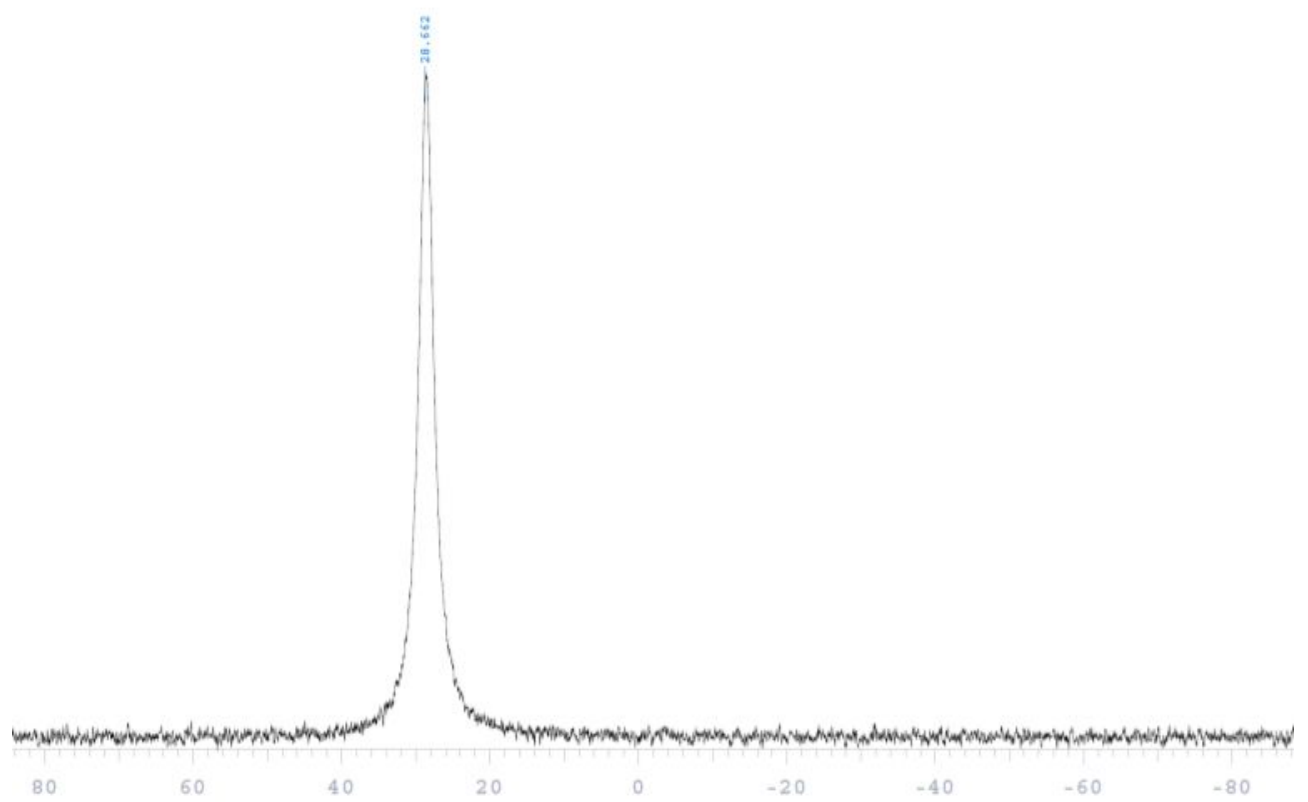




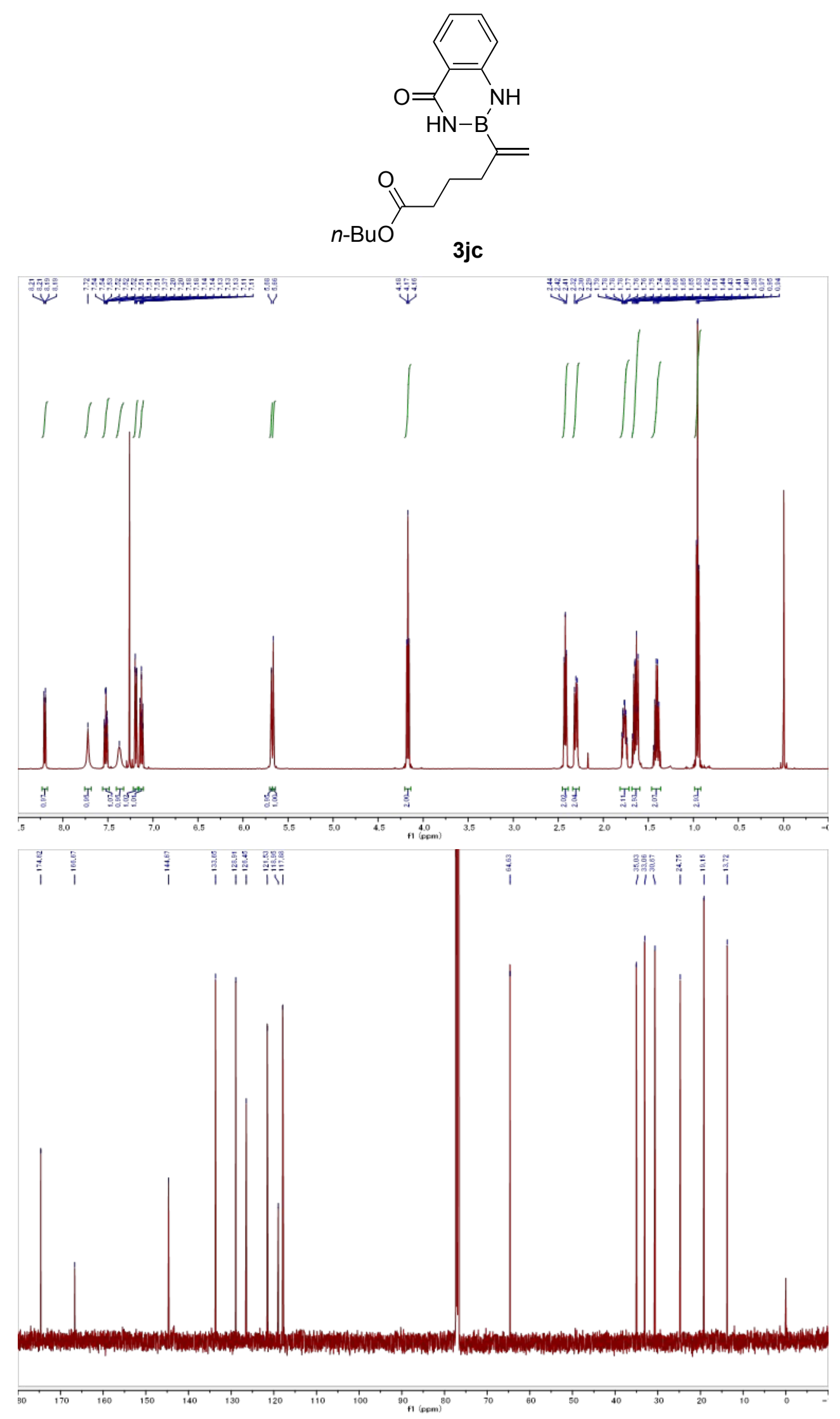




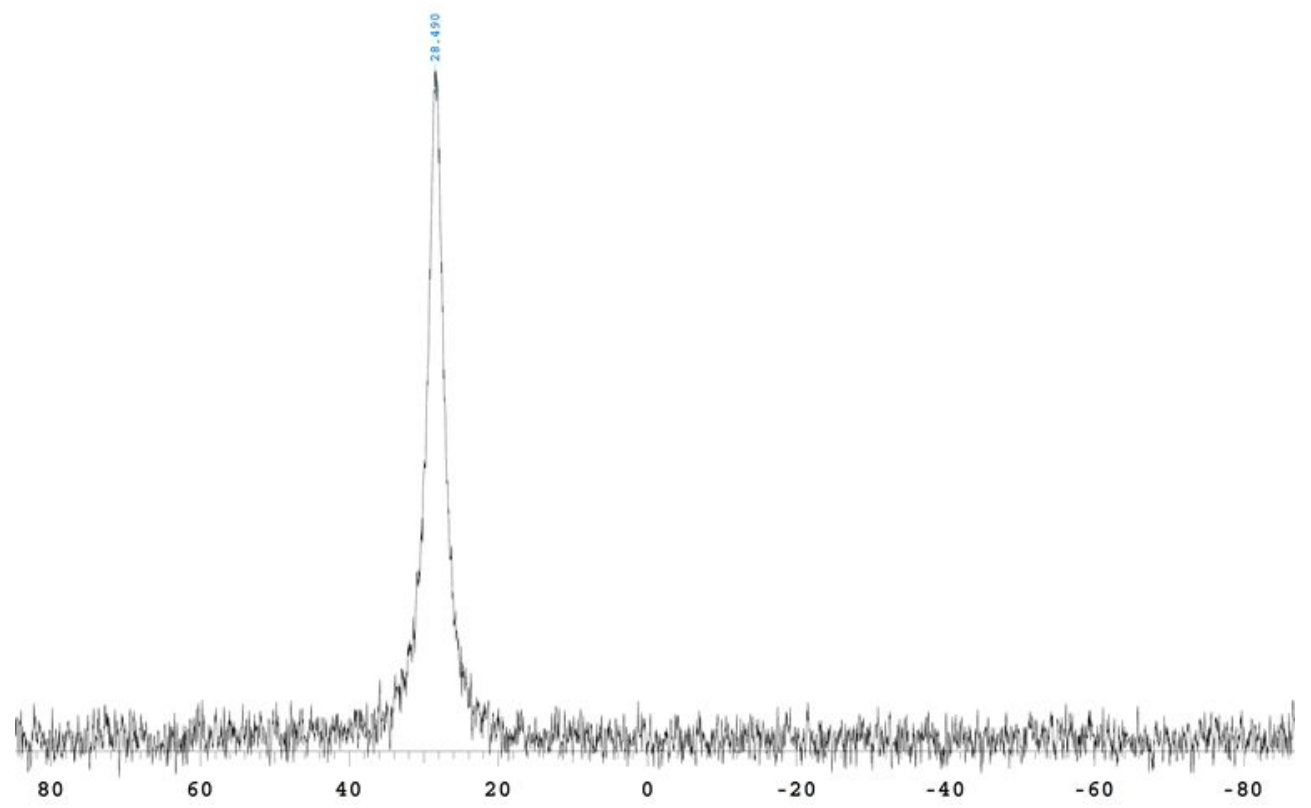




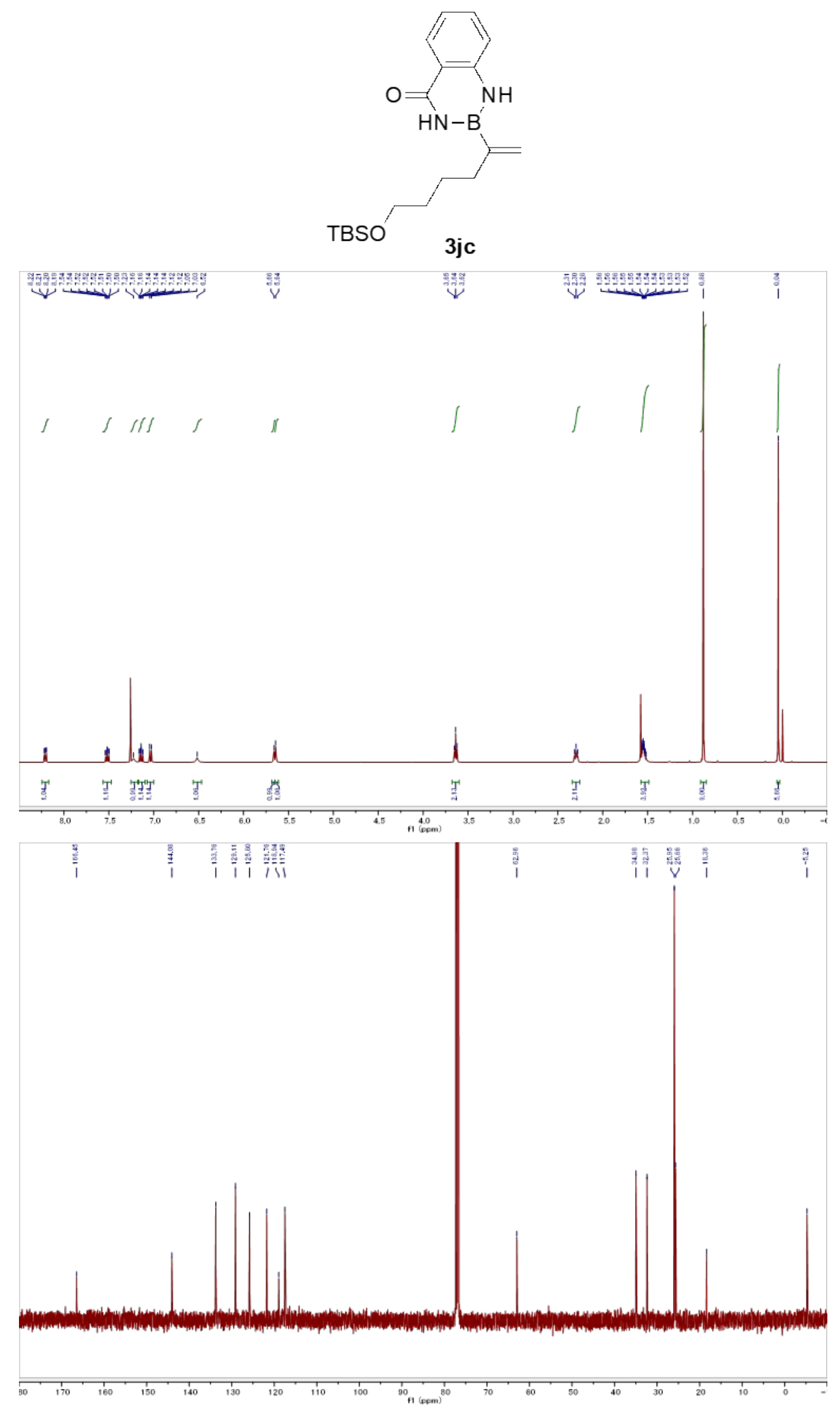




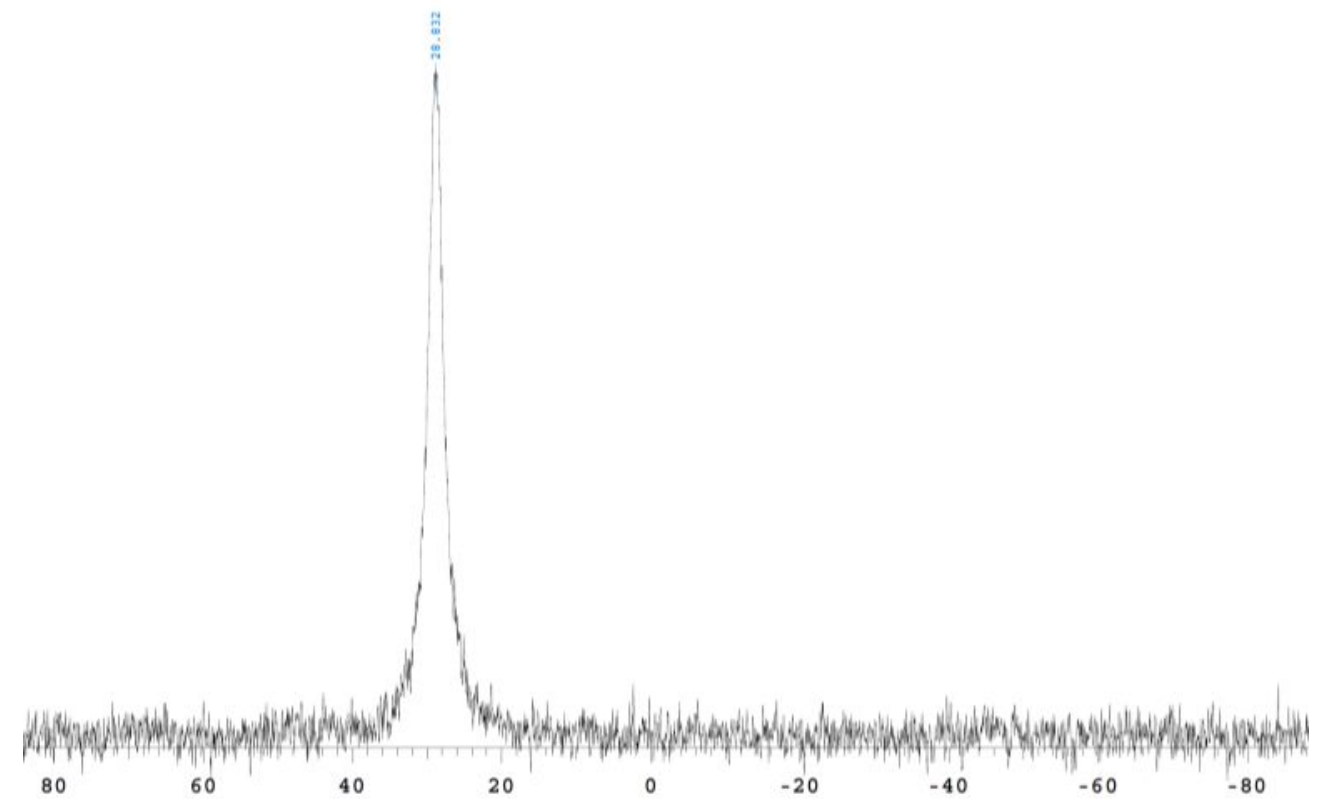




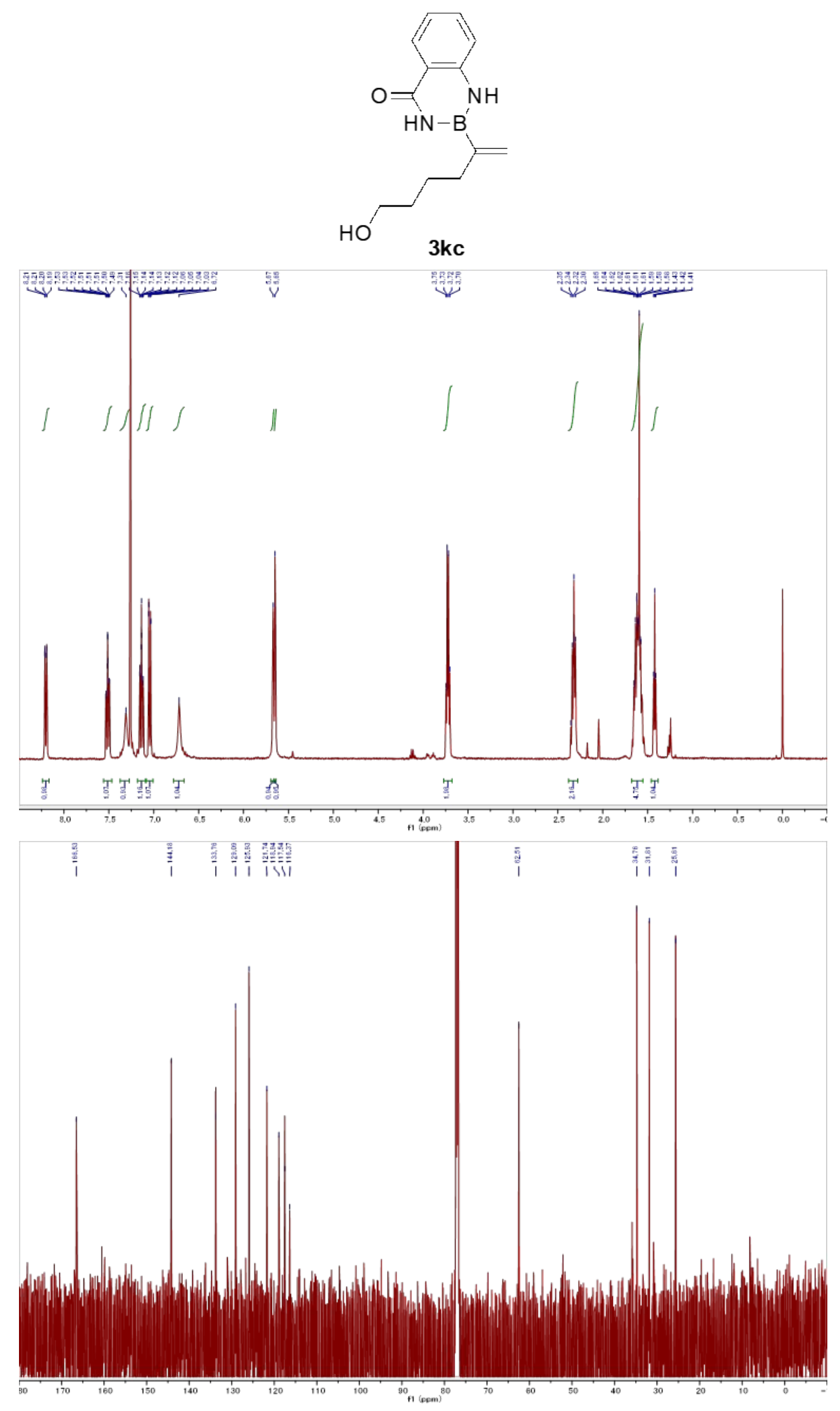




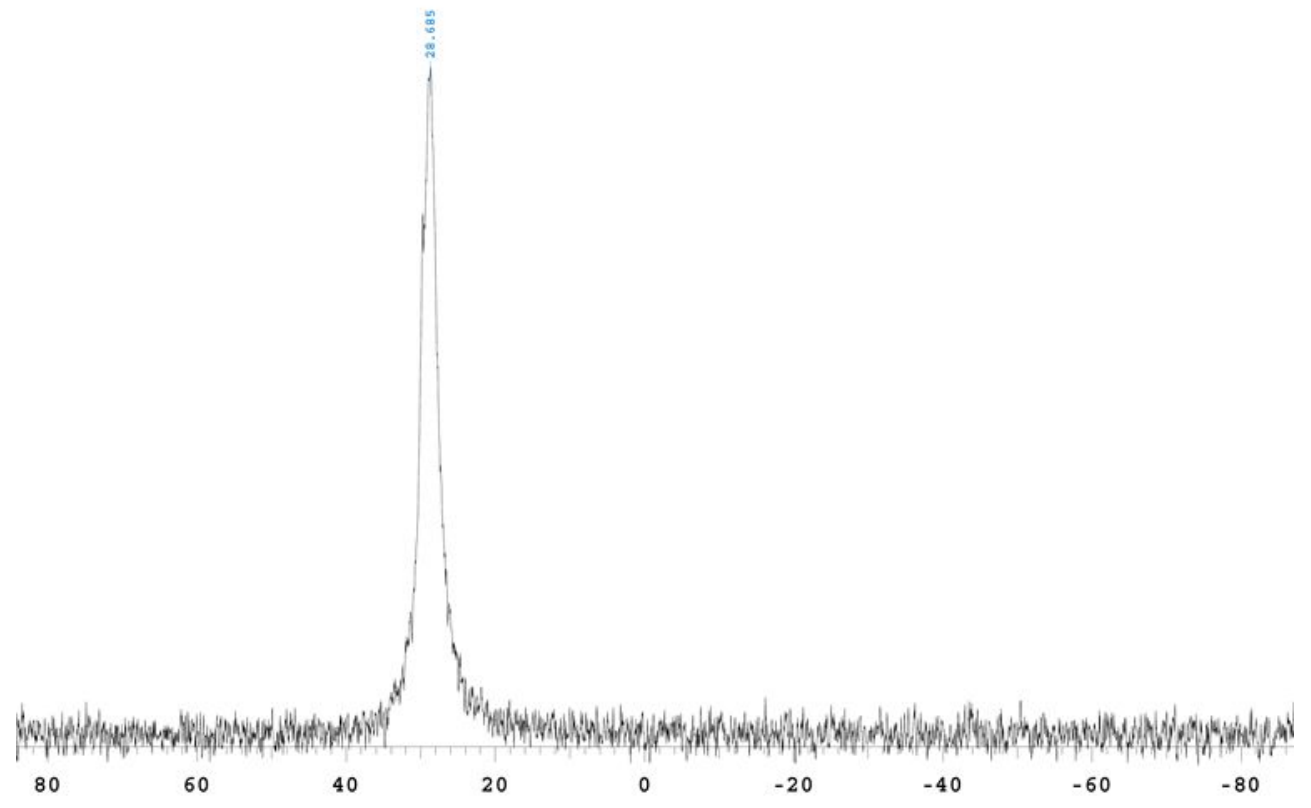



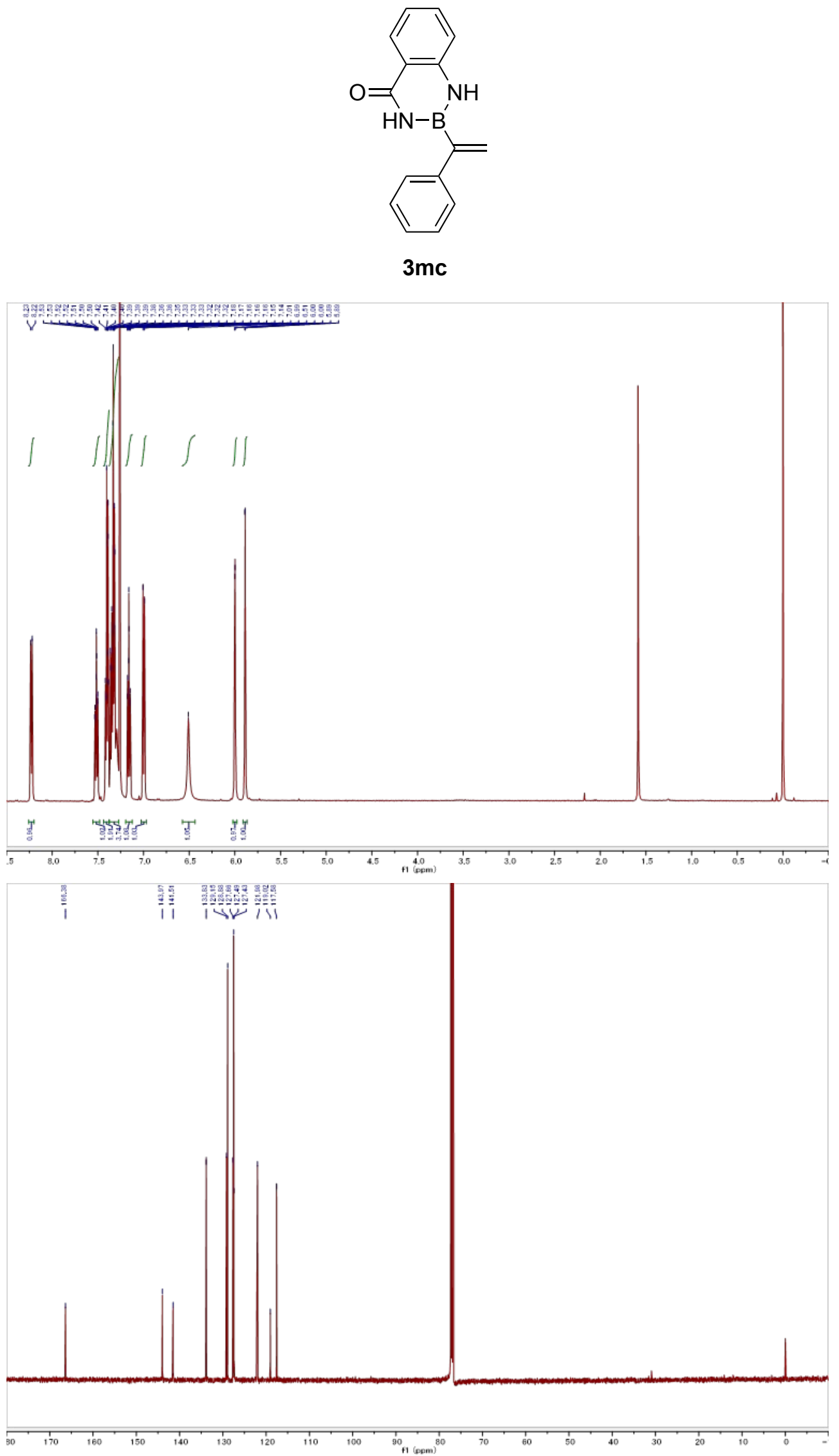


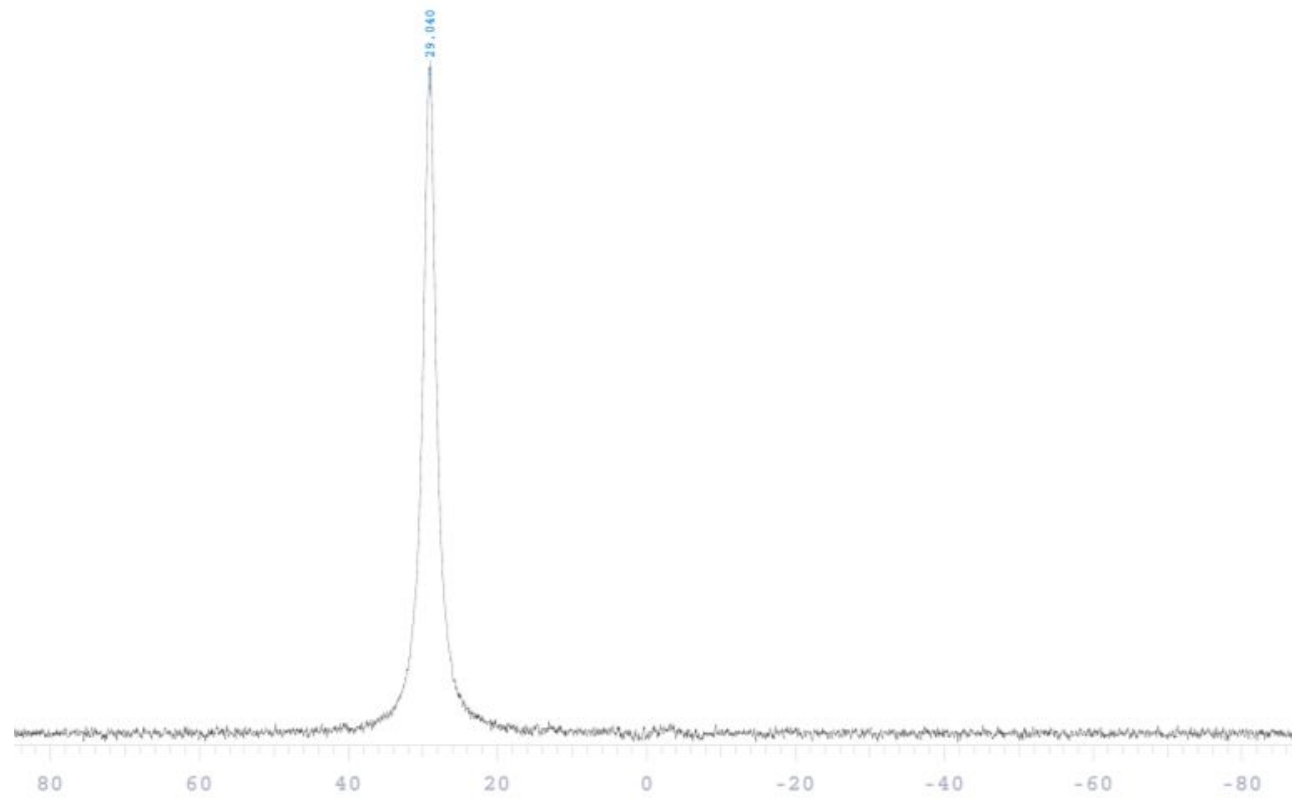



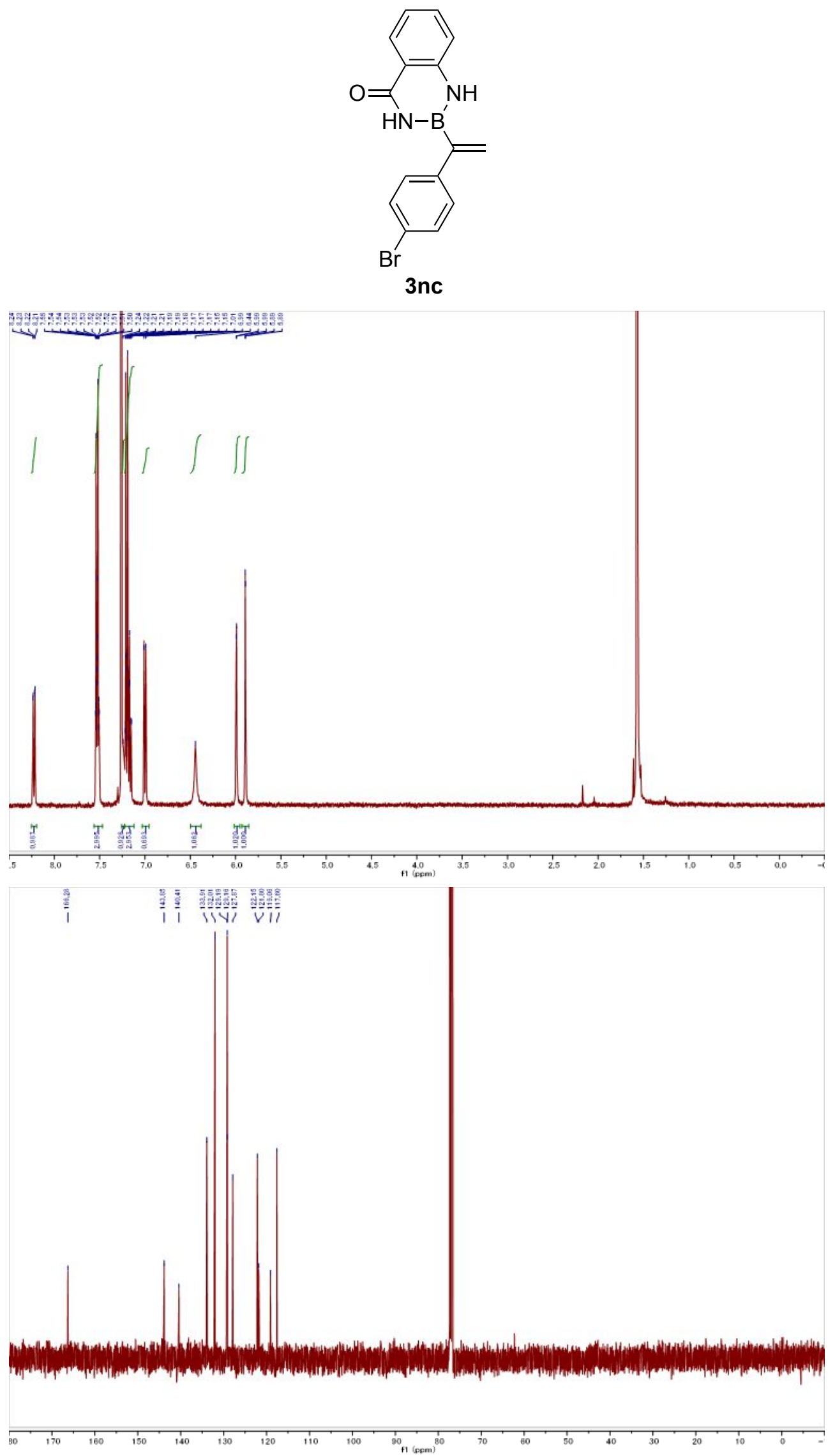


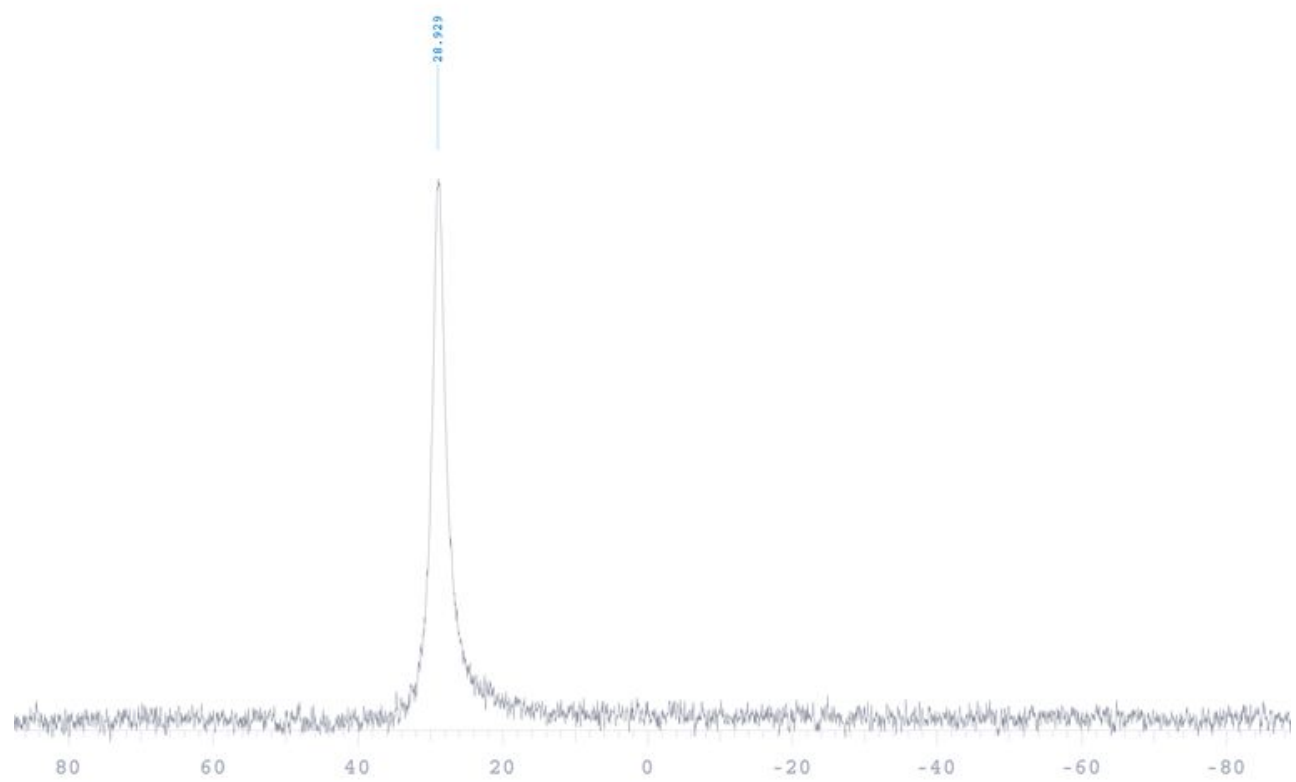



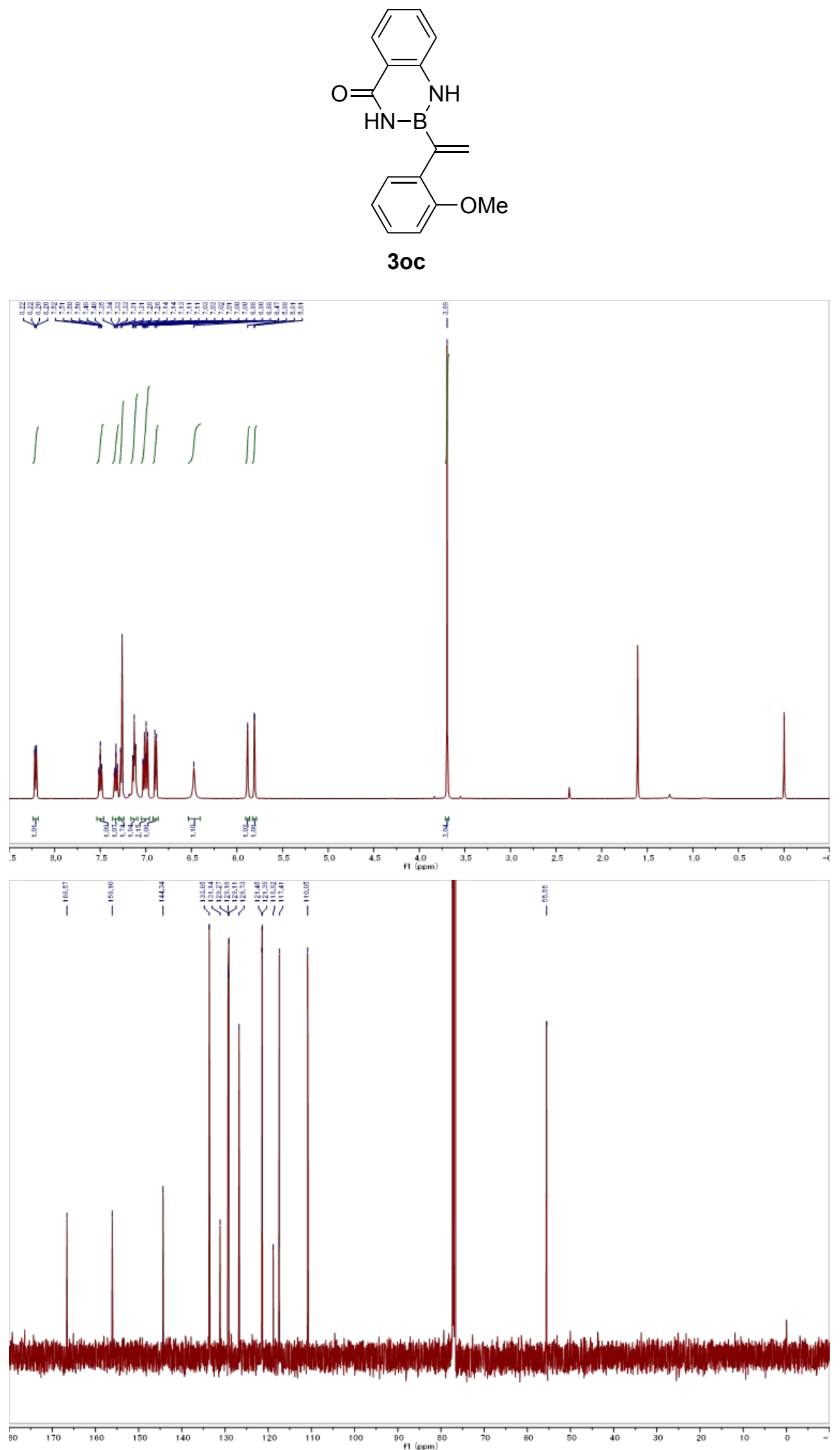


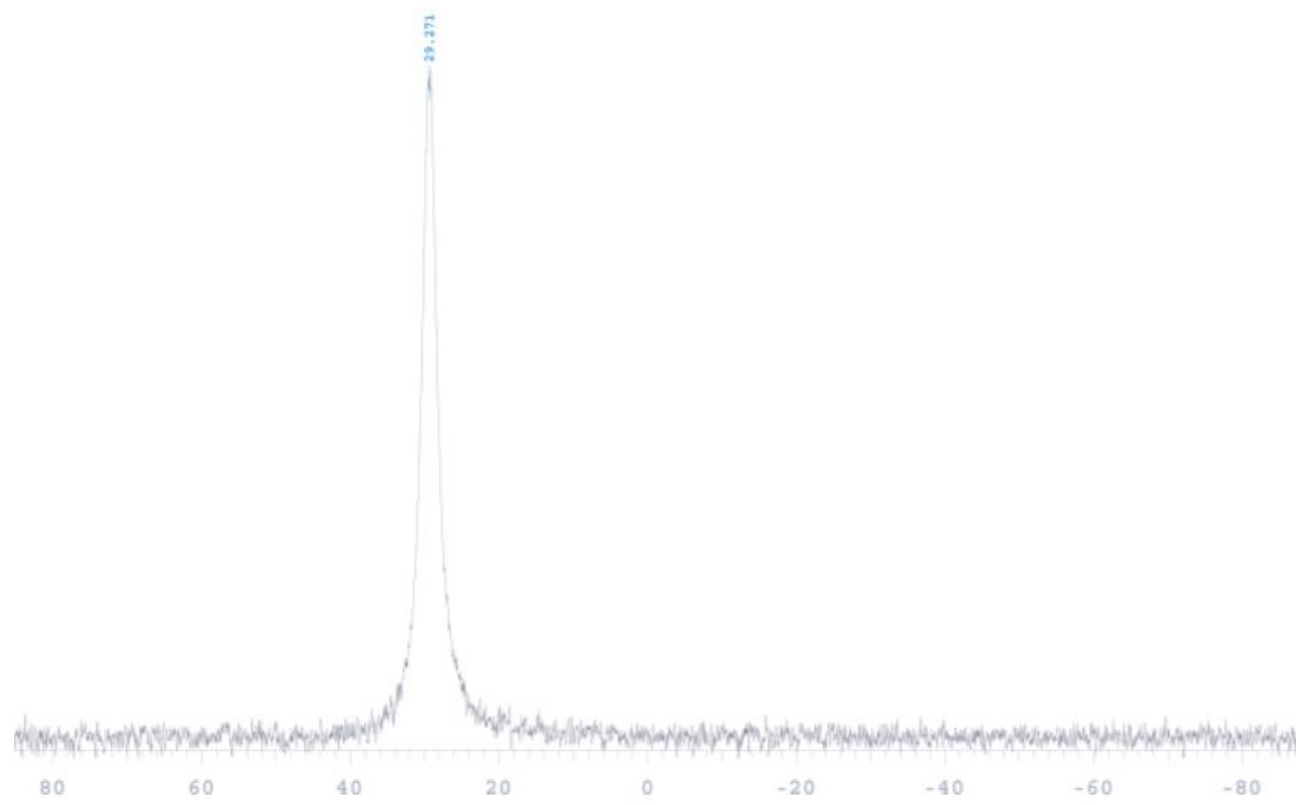



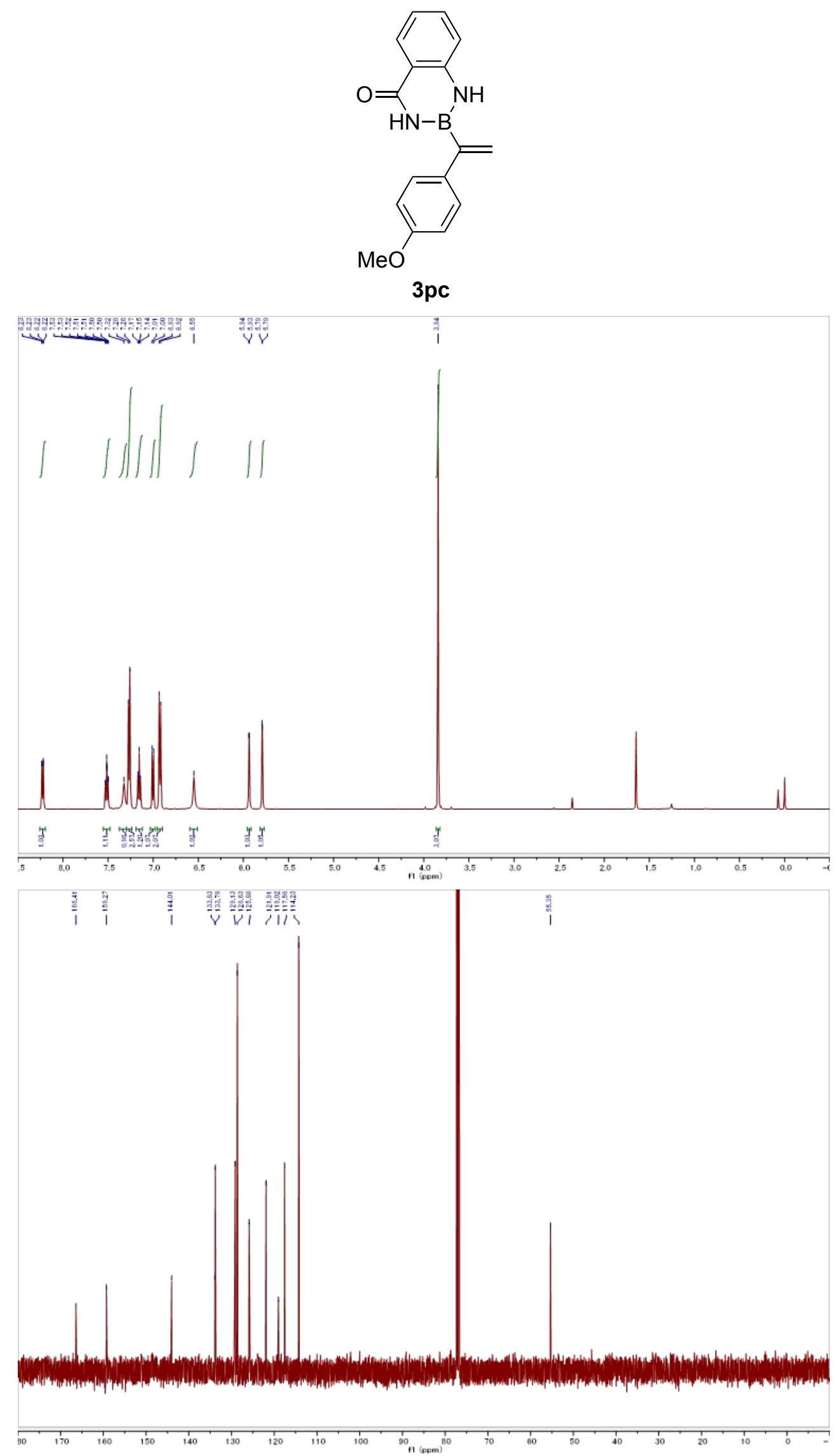


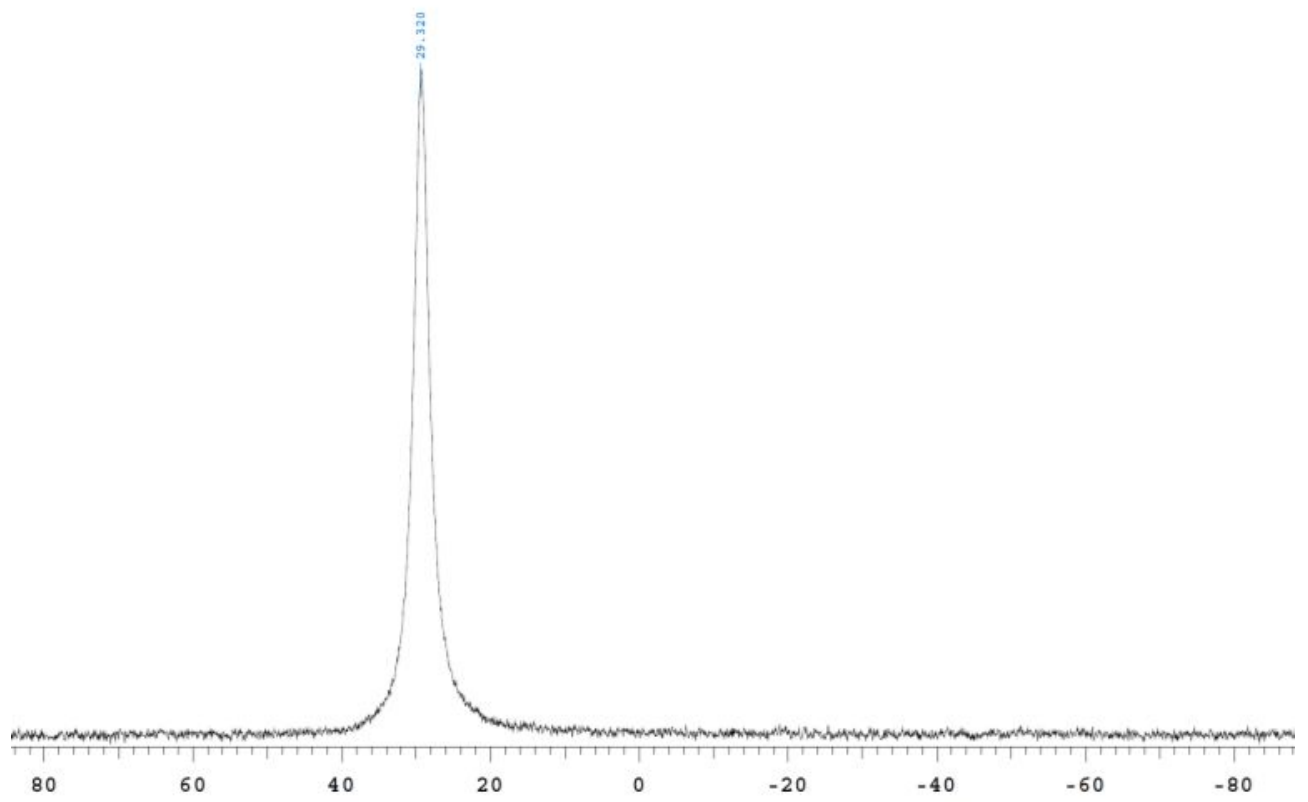




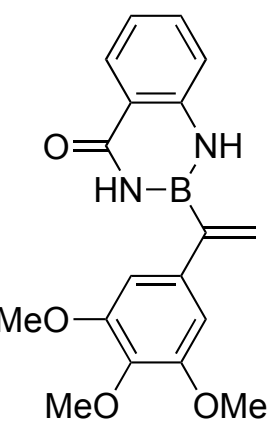

3qc
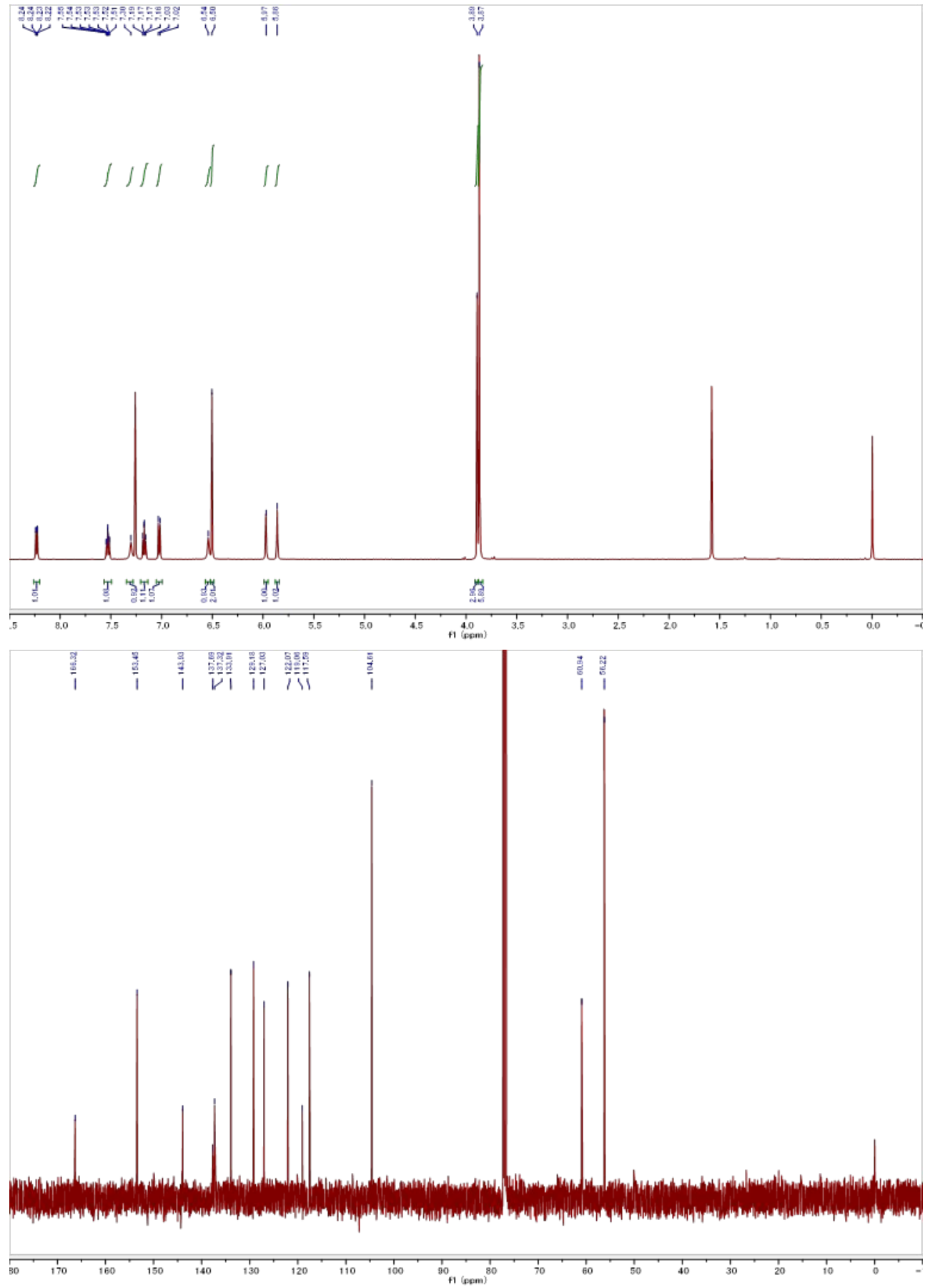


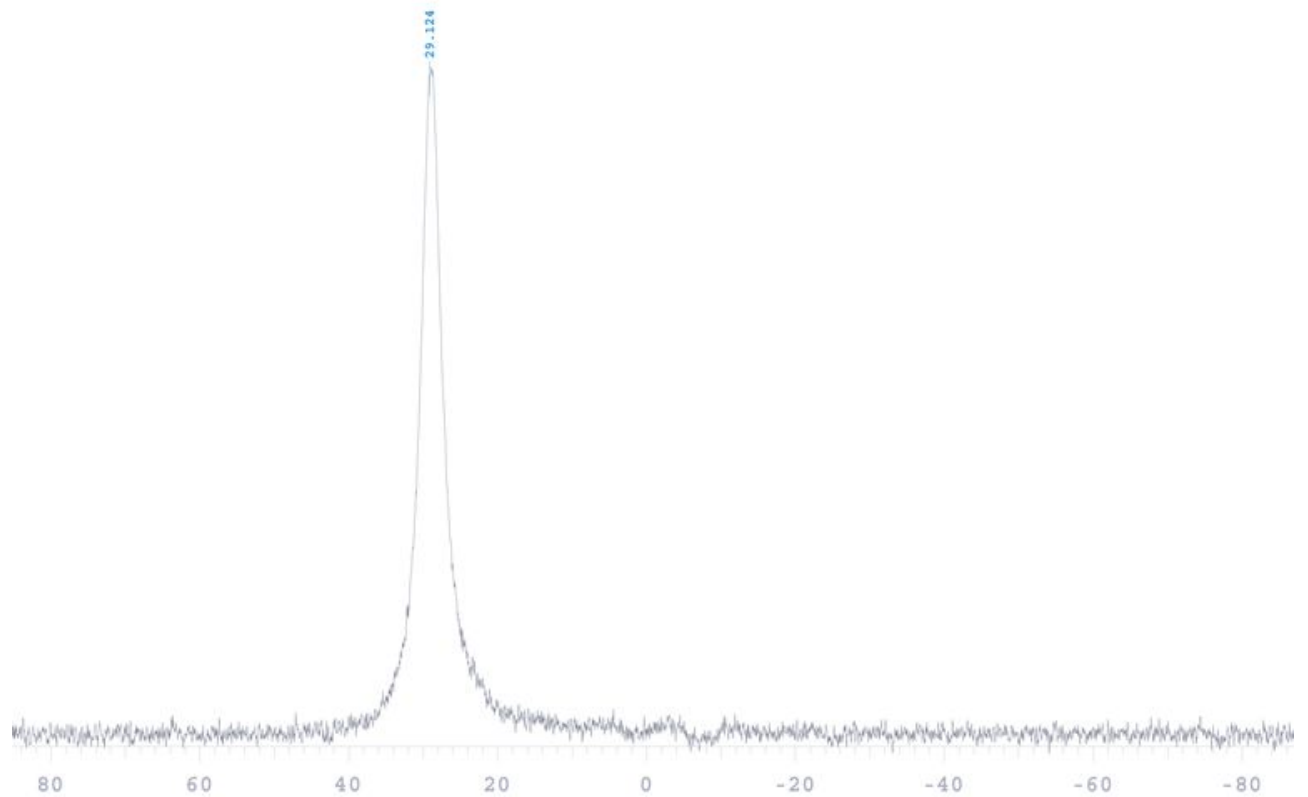



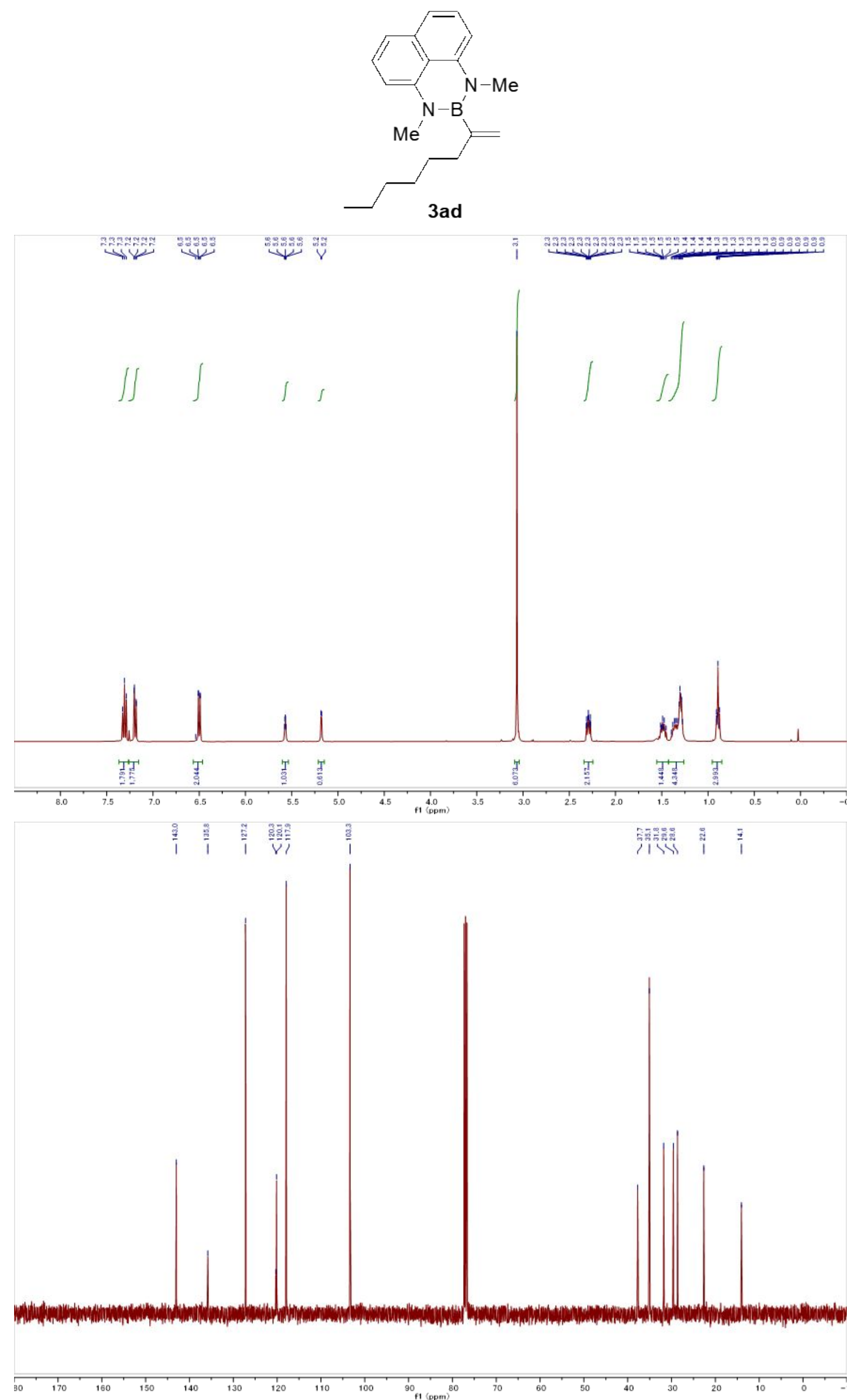


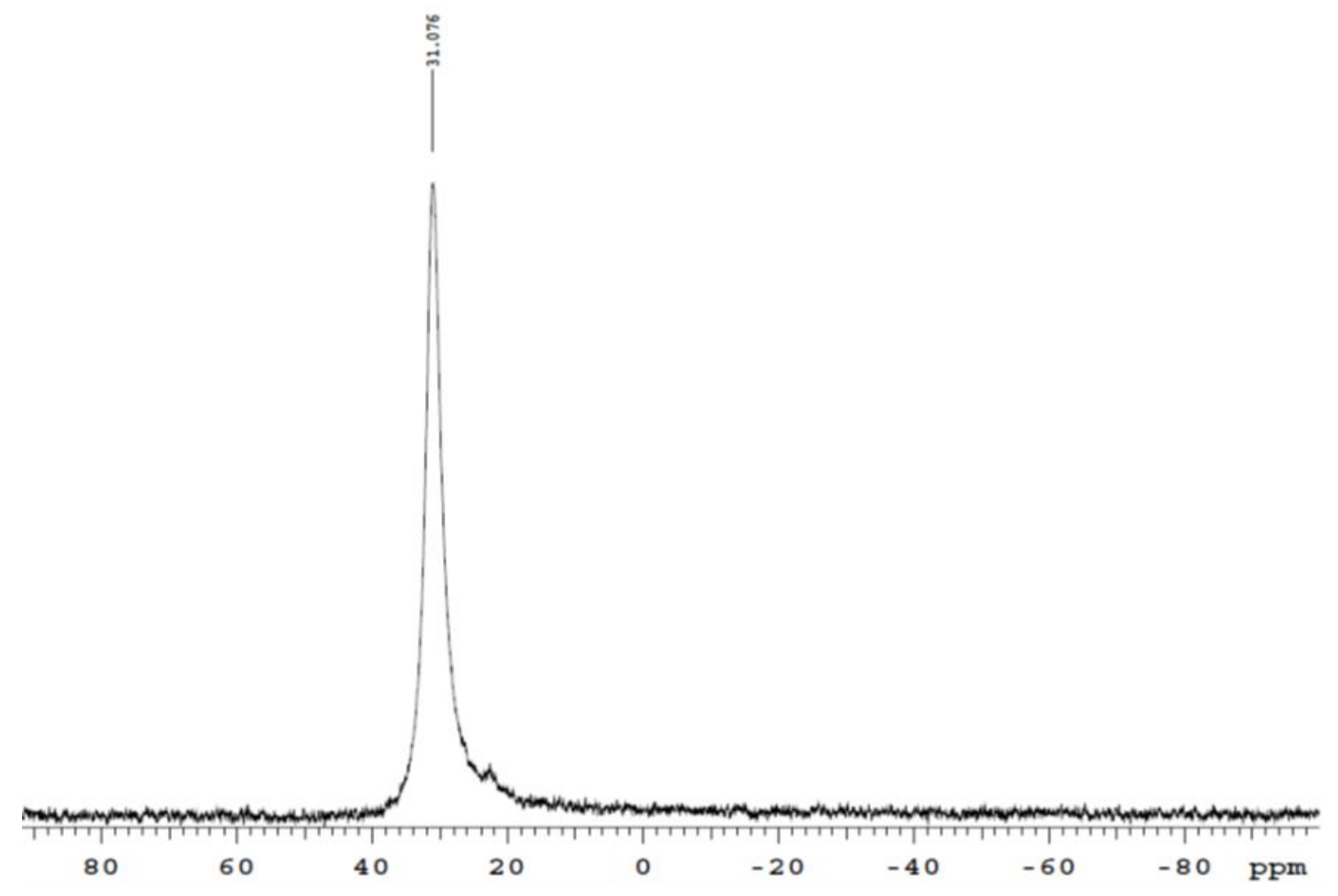




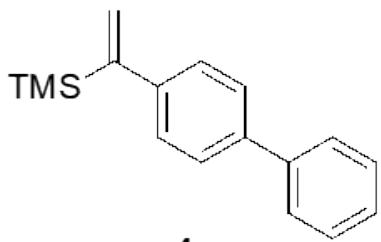

$4 a$
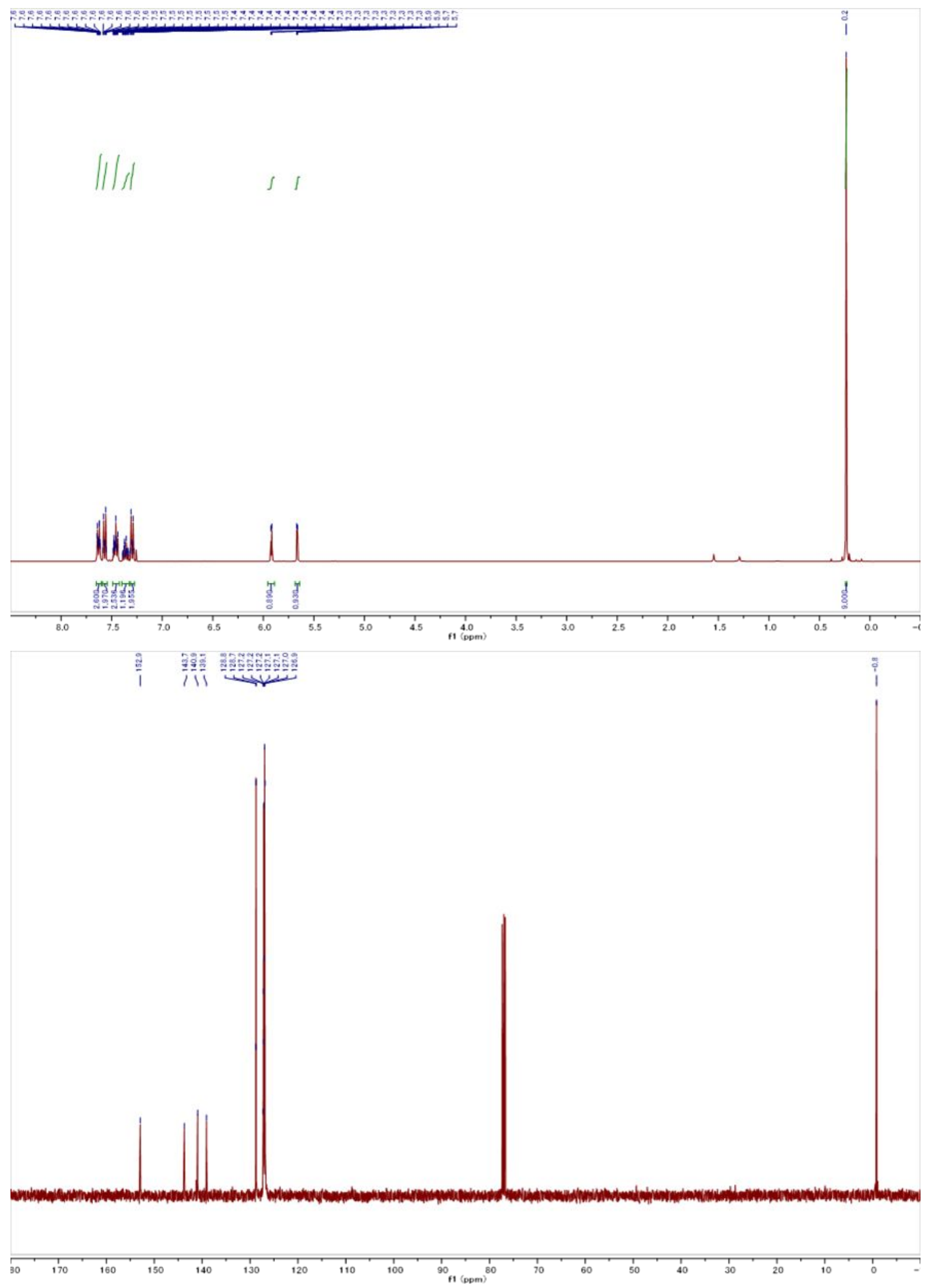

S80 